\title{
Estabilidade vertical no problema circular de Sitnikov
}

\author{
Marcelo Farias Caetano
}

\author{
DisSERTAÇÃO APRESENTADA \\ $\mathrm{AO}$ \\ Instituto de Matemática e Estatística \\ DA \\ Universidade DE SÃo PAUlo \\ PARA \\ OBTENÇÃO DO TÍTULO \\ $\mathrm{DE}$ \\ Mestre em Matemática Aplicada
}

\author{
Programa: Matemática Aplicada \\ Orientador: Prof. Dr. Manuel Valentim de Pera Garcia
}

Durante o desenvolvimento deste trabalho o autor recebeu auxílio financeiro da CNPq

São Paulo, Dezembro de 2011 


\section{Estabilidade vertical no problema circular de Sitnikov}

Este exemplar corresponde à redação final da dissertação devidamente corrigida e defendida por Marcelo Farias Caetano e aprovada pela Comissão Julgadora.

Esta versão definitiva da dissertação contém as correções e alterações sugeridas pela Comissão Julgadora durante a defesa realizada por Marcelo Farias Caetano em 19/12/2011.

Comissão Julgadora:

- Profa. Dr. Manuel Valentim de Pera Garcia (orientador) - IME-USP

- Prof. Dr. Clodoaldo Grotta Ragazzo - IME-USP

- Prof. Dr. Sylvio Ferraz-Mello - IAG-USP 
Aos meus pais 


\section{Agradecimentos}

Agradeço aqui,

Aos meus pais, Fábio e Maria de Fátima, pelo apoio, suporte e amor em todos os momentos da minha vida.

À minha família, principalmente às minhas irmãs, avós e tia por me aguentarem e pelo apoio dado durante esses anos.

Principalmente, aos professores Manuel Valentim de Pera Garcia pela difícil tarefa de me orientar e ensinamentos durante todo o trabalho, e ao professor Clodoaldo Grotta Ragazzo pela discussões construtivas sobre matemática, ensinamento e apoio durante a elaboração desse trabalho. Aos professores Ângelo Barone Netto, Sônia Regina Leite Garcia, Ricardo Freire pelas dicas e correções dadas ao trabalho, e discussões calorosas durante os seminários dados por mim para a elaboração deste trabalho.

Aos amigos do IMEUSP Alexandre Abdo, Danilo Tonini, Eduardo Oda, Ricardo Ribeiro, Thiago Araújo, e tantos outros com os quais eu pude contar com o apoio. Aos amigos do futebol do IMEUSP, o qual me servia como um escape do stress durante o trabalho.

Aos membros da banca, Manuel Valentim Pera Garcia, Clodoaldo Grotta Ragazzo e Sylvio Ferraz-Mello, por terem aceito a realizar a banca em um período pouco convencional.

A Deus, por estar sempre presente.

A CNPQ, pelo apoio financeiro. 


\section{Resumo}

Estudamos um caso especial do problema restrito dos três corpos, chamado problema circular de Sitnikov, quando dois corpos de massas iguais (chamadas de primárias) estão em uma órbita circular (configuração central de dois corpos), enquanto que um terceiro corpo de massa negligenciada (chamada infinitesimal) oscila sobre uma reta perpendicular ao plano das primárias (chamaremos esse movimento de vertical periódico). Aqui analisamos a estabilidade desse movimento periódico, com relação a pequenas perturbações nas direções ortogonais a reta onde ocorre o movimento. Chamaremos a atenção ao fenômeno de alternância entre estabilidade e instabilidade na família do movimento periódico vertical, conforme variamos a amplitude do movimento.

Palavras-chave: Problema de Sitnikov, Estabilidade, Movimentos Periódico. 


\section{Abstract}

We studied a special case of the restricted three-body problem, named circular problem of Sitnikov, when two body of equal mass (called primaries) moving around each other on circular motion (central configuration of two body), while the third body of negligible mass (called infinitesimal) performs along a straight line orthogonal to the plane of the primaries (so called periodic vertical motions). We analyze the stability of the periodic vertical motions with respect to small perturbations orthogonal to the straight line where the motions occurs. We call attention to the phenomenom of alternation of stability and instability within the family of periodic vertical motions, whenever their amplitude is varied in a continuous manner.

Keywords: Sitnikov Problem, Stability, Periodic Motions. 


\section{Sumário}

Lista de Figuras $\quad$ xi

Lista de Tabelas $\quad$ xiii

1 Introdução $\quad 1$

1.1 Descrição do problema . . . . . . . . . . . . . . . . . 2

1.2 Considerações Preliminares . . . . . . . . . . . . . . . . . . 3

1.3 Organização do Trabalho . . . . . . . . . . . . . . . . . . . . . . 3

2 Sistemas Hamiltonianos $\quad \mathbf{5}$

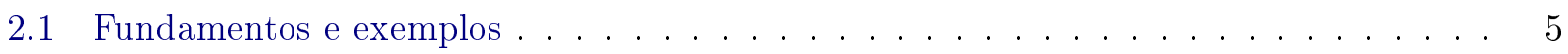

2.2 Sistemas de equações diferenciais lineares $\ldots \ldots \ldots \ldots \ldots$

2.2 .1 Definições básicas . . . . . . . . . . . . . . . . . . 8

2.2.2 Sistemas de equações diferenciais lineares com coeficientes periódicos arbitrários 10

2.3 Sistemas Hamiltonianos e Canônicos de Equações Diferenciais Lineares . . . . . . . . 12

2.3 .1 Definições básicas . . . . . . . . . . . . . . . . . . . . . 12

2.3.2 Propriedades de soluções das equações canônicas e Hamiltonianas . . . . . . . 14

2.3 .3 Teorema de Lyapunov-Poincaré . . . . . . . . . . . . . . . . . . . . . . 15

2.3.4 Equações variacionais de Poincaré e suas propriedades . . . . . . . . . . . 16

3 Descrição do problema restrito $r$

3.1 Introdução . . . . . . . . . . . . . . . . . . . . . . . . . . . 19

3.2 Descrição do problema planar . . . . . . . . . . . . . . . . . 19

3.2.1 Definição do problema e as equações do movimento no sistema inercial . . . . 19

3.2.2 Equações do movimento no sistema de coordenadas sinódicas e a integral Jacobiana . . . . . . . . . . . . . . . . . . . . . . . 21

3.2.3 Equações do movimento em coordenadas não físicas . . . . . . . . . . . . 23

3.2.4 Relação com o problema geral dos três corpos . . . . . . . . . . . . . . 25

3.2.5 Classificação e modificações no problema restrito . . . . . . . . . . . 28

3.3 Descrição do problema espacial . . . . . . . . . . . . . . . . . . . . 29

3.3.1 Formulação do problema e equação do movimento . . . . . . . . . . . . 29

3.4 Configurações centrais . . . . . . . . . . . . . . . . . . . . 31

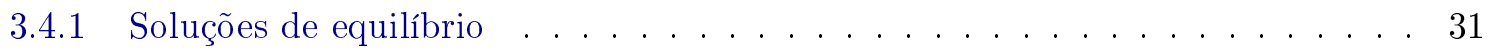

3.4 .2 Equações para a configuração central . . . . . . . . . . . . . . . . . . 31

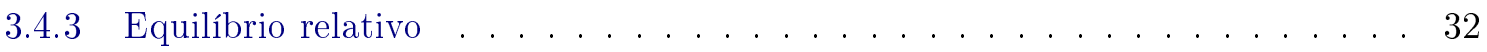


3.5 Descrição do problema restrito dos $(N+1)$ corpos $\ldots \ldots \ldots \ldots \ldots$

4 Alternância de estabilidade e instabilidade na família do movimento vertical 35

4.1 Generalidades do problema . . . . . . . . . . . . . . . . 36

4.2 Aproximação da matriz de monodromia . . . . . . . . . . . . . . . . . 38

4.2 .1 Propriedades do sistema linear . . . . . . . . . . . . . . . . . 38

4.2 .2 Expressão aproximada da matriz de monodromia . . . . . . . . . . . . . . . 39

4.3 Resultados numéricos . . . . . . . . . . . . . . . . . . . . 45

4.3.1 Resultados para $5<a<13 \ldots \ldots \ldots \ldots \ldots \ldots$

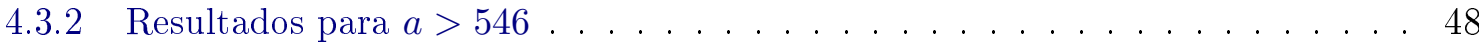

5 Conclusões $\quad 51$

5.1 Considerações Finais . . . . . . . . . . . . . . . . . . . . . . 51

A Funções matriciais e logaritmo de matriz

A.1 Funções matriciais . . . . . . . . . . . . . . . . . . . 53

A.1.1 Funções matriciais holomórficas . . . . . . . . . . . . . . . . . 53

A.1.2 Funções matriciais analíticas por partes . . . . . . . . . . . . . . 54

A.1.3 Lema da composição de funções . . . . . . . . . . . . . . . . . . . 55

A.1.4 Logaritmo de Matriz . . . . . . . . . . . . . . . . . . . . . . 56 56

$\begin{array}{lll}\text { B Algoritmos implementados } & 57\end{array}$

$\begin{array}{ll}\text { Referências Bibliográficas } & 65\end{array}$ 


\section{Lista de Figuras}

3.1 Configuração em coordenadas inerciais e rotacionais $\left(m_{1}>m_{2}\right) \ldots \ldots \ldots$. . . 20

3.2 Configuração em coordenadas inercial $(\xi, \eta)$ e rotacional $(x, y)$ nas variáveis não físi-

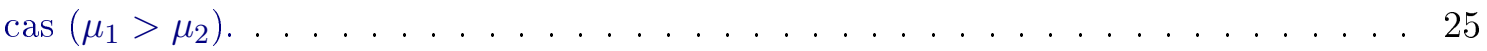

3.3 O problema geral dos três corpos; as massas são $m_{i}$ e os vetores posições $r_{i}$. . . . 26

3.4 O problema restrito tridimensional, e seu pontos de equilíbrio $L_{i}$. . . . . . . . . . 29

4.1 Retrato de fase na variedade $\tilde{\nu}$. Linha mais escura é a separatriz do espaço $(\tilde{\mathcal{H}}=0) . \quad 37$

4.2 Gráfico da função $(4.4) \ldots \ldots \ldots \ldots$. . . . . . . . . . . . . . 41

4.3 Trajetória realizada pela massa infinitesimal no espaço para a amplitude inicial de $5.0500 \ldots \ldots \ldots \ldots \ldots \ldots$

4.4 Trajetória realizada pela massa infinitesimal no espaço para a amplitude inicial de $5.0525 \ldots \ldots \ldots \ldots \ldots \ldots \ldots$

4.5 Trajetória realizada pela massa infinitesimal no espaço para a amplitude inicial de $5.4600 \ldots \ldots \ldots \ldots \ldots \ldots \ldots \ldots \ldots$

4.6 Trajetória realizada pela massa infinitesimal no espaço para a amplitude inicial de $5.4701 \ldots \ldots \ldots \ldots \ldots \ldots \ldots \ldots \ldots$

4.7 Comportamento dos índices de estabilidade na vizinhança de onde ocorre as primeiras "selas complexas"(região em branco no gráfico). . . . . . . . . . . . . . . . . . . . 49

4.8 Comportamento dos índices de estabilidade na vizinhança de onde ocorre as primeiras "selas complexas"(região em branco no gráfico) . . . . . . . . . . . . . . . . . 50 


\section{Lista de Tabelas}

2.1 características das soluções de acordo com o expoente ou multiplicadores do sistema

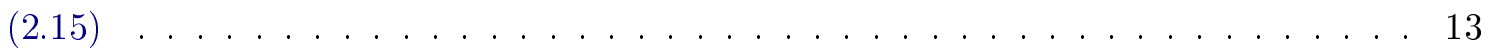

4.1 Intervalos de estabilidades para o parâmetro $a$ do problema de Sitnikov, [SBD07]. Os limites inferior e superior são os bordos do intervalo de estabilidade. Fora desse intervalo o movimento é instável. . . . . . . . . . . . . . . . . . . . 47

4.2 Valores das amplitudes (adimensional) inicial e respectiva estabilidade do movimento periódico vertical em que ela se encontra e os valores dos índices de estabilidade. . . 50 


\section{Capítulo 1}

\section{Introdução}

O problema dos $N$ corpos refere-se à descrição do o movimento de $N$ pontos materiais ou partículas sob influência da lei de movimento de Newton, onde as únicas forças atuantes são as forças de atração gravitacional entre as mesmas. O sistema de equações diferenciais ordinárias que descreve o problema pode ser resolvido por quadratura para $N=2$, pois pode ser reduzido ao problema de Kepler (ver exemplo 2 da seção 2.1).

Ao estudar o movimento de Marte, Newton resolveu completamente o problema dos 2 corpos. Em primeira aproximação, a órbita de Marte é solução das equações do problema, onde somente as forças gravitacionais do Sol e de Marte são levadas em conta, e esse problema pode ser reduzido ao problema de Kepler. Usando métodos da teoria de pertubarções, ele foi capaz de estimar alguns dos efeitos de ordem superior e descrever algumas anomalias da órbita de Marte.

Depois disso, Newton começou a olhar para o problema da órbita da lua. Esse problema embora parecesse simples, mostrou-se muito mais difícil que o problema da órbita de Marte, pois sua primeira aproximação é o problema dos 3 corpos (Terra, Lua e Sol). Hoje em dia a órbita da Lua é estimada através de integração numérica ou por expansão assintótica.

Por causa das dificuldades encontradas já no problema dos 3 corpos, hoje é apoiada amplamente a crença de que o problema dos $N$ corpos seja não integrável para $N \geq 3$. Entretanto há uma grande quantidade de artigos sobre o assunto que falam sobre soluções especiais, estimativas assintóticas, informações sobre colisões, existência ou não de integrais, soluções em séries, singularidades, etc...

A existência, estabilidade e bifurcação de soluções periódicas do problema dos $N$ corpos têm sido assuntos de vasta literatura, particulamente após os trabalhos de Poincaré que estudou extensivamente sobre as soluções periódicas, merecendo destaque seu notável Les méthodes nouvelles de la mécanique céleste, reservado a este tópico. Há uma quantidade vasta de literatura sobre a existência e natureza do movimento periódico do problema dos $N$ corpos, especialmente em um caso particular, chamado de problema restrito.

O problema restrito dos $N$ corpos, é uma "aproximação"do problema geral. Isto é, supomos que $k<N$ corpos não influenciam no movimento dos outros $N-k$ corpos e os movimentos um dos outros, para isso definimos as massas desses $k$ corpos como sendo "nulos", no sentido de serem muito menores que as massas dos outro $N-k$ corpos, motivo do qual chamamos esse problema como uma aproximação do problema geral. Além disso supomos que os $k$ corpos estejam em alguma configuração central, com solução conhecida (solução do problema dos $k$ corpos), para maiores detalhes conferir o capítulo sobre descrição do problema restrito.

Aqui detalharemos um dos métodos usados na análise do movimento periódico, em particular no estudo de sua estabilidade orbital. Estudaremos o chamado problema de Sitnikov circular, um caso particular do problema dos 3 corpos restrito (problema dos 3 corpos em que a força gravitacional de um dos corpos não influência os outros dois corpos).

O termo "problema de Sitnikov"refere-se à seguinte situação: dois corpos de massas iguais, que chamaremos de primárias, com órbitas elíticas coplanares, cofocais, com o foco comum localizado em seu baricentro $O$, enquanto um terceiro corpo de massa infinitesimal, chamado de corpúsculo ou simplesmente infinitesimal, move-se ao longo da reta perpendicular ao plano das primárias que 
passa por $O$, e pretende-se estudar os movimentos da infinitesimal.

Sitnikov, [Sit60] estudou o que hoje é conhecido como "problema elíptico", em que a configuração central das primárias formam duas elipses disjuntas confocal, a qual aparece o chamado movimento oscilatório. O caso em que as primárias movem-se em uma órbita circular já fora estudado anteriormente por Pavanini (1907) e posteriormente por MacMillan (1911). Nesta situação o problema fica muito mais simples, pois torna-se intergrável e é possível obter sua solução em termos das funções elíticas de Jacobi. Embora o problema circular tenha sido "apresentado"antes dos estudos de Sitnikov, o termo popular para ele ficou sendo Problema de Sitnikov circular, embora alguns autores o chamem de "problema de Pavanini"ou "problema de MacMillan".

\subsection{Descrição do problema}

Após darmos um breve histórico sobre o problema restrito e sua relação com o problema geral, neste parágrafo resumiremos o problema que iremos estudar em detalhes no capítulo (4).

Considere o problema restrito circular com primárias de massas $m_{1}=m_{2}=m$. Seja $O x_{1} x_{2} x_{3}$ coordenadas sinódicas (maiores detalhes ver capítulo (3)), com origem, $O$, localizada no baricentro das primárias; as primárias $\mathcal{P}_{1}$ e $\mathcal{P}_{2}$ arranjadas de tal forma que a reta que as une será o eixo $O x_{1}$, e elas realizam rotação em torno do eixo $O x_{3}$. As coordenadas adimensionais da infinitesimal serão

$$
q_{1}=x_{1}, q_{2}=x_{2}, q_{3}=x_{3} .
$$

A hamiltoniana para as equações do movimento da infinitesimal é

$$
H=\frac{1}{2}\left(p_{1}^{2}+p_{2}^{2}+p_{3}^{2}\right)+p_{1} q_{2}-p_{2} q_{1}-\frac{1}{2}\left(\frac{1}{r_{1}}+\frac{1}{r_{2}}\right),
$$

em que $r_{1}$ e $r_{2}$ denotam as distâncias entre a infinitesimal e as respectivas primárias, $p_{1}, p_{2}, p_{3}$ são os momentos conjugados de $q_{1}, q_{2}, q_{3}$.

O espaço de fase, onde está definido o movimento da infinitesimal possui uma subvariedade

$$
\tilde{\Sigma}=\left\{(p, q) ; p_{1}=p_{2}=q_{1}=q_{2}=0\right\}
$$

, que é invariante com respeito ao fluxo.

Chamaremos de movimento vertical trajetórias do corpúsculo pertencentes a $\tilde{\Sigma}$, ou seja, o movimnto de 3 no eixo $O x_{3}$.

A um movimento periódico nessa variedade podemos associar um parâmetro, $a$, chamado de amplitude do movimento, e podemos escolher como valor desse parâmetro a amplitude do movimento periódico $\left(a=\max _{t \in \mathbb{R}}\left|q_{3}(t)\right|\right)$, ou o valor absoluto de $p_{3}$ quando a infinitesimal passa pelo baricentro, ou o valor da energia $h$ do movimento periódico.

Dependendo das condições iniciais dadas $\left(\left(p_{0}, q_{0}\right) \in \tilde{\Sigma}\right)$, há três tipos de movimento: escape hiperbólico $\left(\lim _{t \rightarrow \infty}\left|q_{3}\right|=\infty\right.$ e $\left.\lim _{t \rightarrow \infty}\left|p_{3}\right|>0\right)$, escape parabólico $\left(\lim _{t \rightarrow \infty}\left|q_{3}\right|=\infty\right.$ e $\lim _{t \rightarrow \infty}\left|p_{3}\right|=$ $0)$ e movimento periódico vertical $\left(\max _{t \in \mathbb{R}}\left|q_{3}(t)\right|<\infty\right.$.)

Dado um cubo $R=\left[\gamma_{1}, \gamma_{2}\right] \times\left[\beta_{1}, \beta_{2}\right] \times\left[\eta_{1}, \eta_{2}\right] \subset \mathbb{R}^{3}, \alpha=\left(\alpha_{1}, \alpha_{2}, \alpha_{3}\right) \in R$, chamaremos de uma perturbação de uma trajetória periódica $X\left(t, t_{0}, q_{0}\right)=\left(q^{1}\left(t, t_{0}, q_{0}\right), q^{2}\left(t, t_{0}, q_{0}\right), q^{3}\left(t, t_{0}, q_{0}\right)\right)$ a trajetória $X_{\alpha}\left(t, t_{0}, q_{0}\right)=\left(q_{1}\left(t, t_{0}, q_{0}+\alpha\right), q_{2}\left(t, t_{0}, q_{0}+\alpha\right), q_{3}\left(t, t_{0}, q_{0}+\alpha\right)\right)$.

Chamaremos de estabilidade do movimento vertical, $X\left(t, t_{0}, q_{0}\right)$ se $\forall \alpha \in R$ a trajetória $X_{\alpha}\left(t, t_{0}, q_{0}\right)$ é limitada. Aqui estudaremos um fenômeno interessante que ocorre no movimento vertical do problema restrito, que é a alternância entre estabilidade e instabilidade dos movimentos periódicos verticais com relação ao parâmetro $a$, isto é, dado um retângulo $R$ suficientemente pequeno, $\forall \alpha \in R$ a trajetória $X_{\alpha}(t, a)$ alterna entre estável e instável. No caso em que $a$ é suficientemente grande o comportamento dessa alternância torna-se ainda mais interessante, pois, para $a>>1$, aparece uma propriedade importante no movimento vertical: o intervalo de instabilidade "sela complexa", em que todos os autovalores da matriz de monodromia são complexos e não estão sobre o círculo 
unitário, de acordo com cálculos numéricos, o primeiro valor do intervalo onde isso ocorre é para $a \approx 546$. Pode-se, então, mostrar numericamente que o fenômeno de alternância nesta família ocorre infinitas vezes e depende continuamente de $a$.

A primeira vez em que essa alternância foi investigada foi em [PM88], mas obtiveram uma conclusão errônea, pois afirmaram que o movimento vertical seria sempre instável. Essa afirmação foi confrontada em 1994 por Belbruno et al. [BLO94], onde a alternância de estabilidade e instabilidade do movimento vertical foi detectada numericamente no caso da variação monótona da amplitude $a$. Na verdade, a "alternância infinita"entre estabilidade e instabilidade de uma família a um parâmetro de soluções periódicas é típica para sistemas Hamiltonianos, porém isso ocorre geralmente em sistemas de dois graus de liberdade.

Entretanto, há uma diferença importante entre o problema de Sitnikov circular e outros tipos de sistemas nos quais ocorre essa alternância. No problema de Sitnikov a família de soluções periódicas tem como limite uma órbita aperiódica ilimitada (movimento de escape parabólico), enquanto nos outros sistemas o limite é uma órbita aperiódica limitada. Devido a essa diferença o problema de Sitnikov circular não pode ser estudado da maneira usual, como em Grotta Ragazzo, C., [GR97].

\subsection{Considerações Preliminares}

Segundo Kenneth R. Meyer, [Mey99], há um velho ditado em mecânica celeste que afirma que "nenhum conjunto de variáveis é bom o bastante". Vários conjuntos de variáveis foram usados até hoje em artigos sobre esse assunto. E não há alfabetos suficientes que permita associar para cada variável um símbolo diferente. Ao introduzirmos o problema dos 3 corpos restrito (seção 3), usaremos alguns sistemas de coordenadas diferentes.

Para a discussão geral usaremos:

$$
z=\left(\begin{array}{c}
q \\
p
\end{array}\right), \quad J=J_{2 n}=\left(\begin{array}{cc}
0 & I_{n} \\
-I_{n} & 0
\end{array}\right), \quad \nabla_{z} H=\nabla H=\left(\begin{array}{c}
\frac{\partial H}{\partial z_{1}} \\
\vdots \\
\frac{\partial H}{\partial z_{2 n}}
\end{array}\right)
$$

Se $A$ for uma matriz, então $A^{T}$ será sua transposta e $A^{*}=\bar{A}^{T}$ (conjugada da transposta ou adjunta) e $A^{-1}$ sua inversa. Chamaremos de $\operatorname{Tr} A$ o traço de $A$ e de $\operatorname{det} A$ seu determinante.

Dizemos que uma função $f: \mathbb{R} \rightarrow \mathbb{C}$ é contínua por partes se ela tem, somente, um número finito de descontinuidade em qualquer intervalo finito.

Uma função contínua por partes integrável tem como propriedade, em qualquer ponto de discontinuidade $t^{*} \in\left(t_{1}, t_{2}\right)$, os limites

$$
\begin{aligned}
& \int_{t_{1}}^{t^{*}}|f(t)| d t=\lim _{\epsilon \rightarrow 0^{+}} \int_{t_{1}-\epsilon}^{t^{*}}|f(t)| d t, \\
& \int_{t^{*}}^{t_{2}}|f(t)| d t=\lim _{\epsilon \rightarrow 0^{+}} \int_{t^{*}+\epsilon}^{t_{2}}|f(t)| d t
\end{aligned}
$$

existem e sempre que $t_{1}, t_{2}$ são escolhidos tais que os intervalos $\left(t_{1}, t^{*}\right),\left(t^{*}, t_{2}\right)$ não contenham outros pontos de descontinuidade.

No caso de matrizes, uma função matricial (ou simplesmente "matriz") é contínua, contínua por partes, integrável, etc. se as condições são satisfeitas para seus coeficientes. Os pontos de descontinuidade da matriz são os pontos de descontinuidade de cada coeficiente. Integrabilidade da matriz implica integrabilidade de sua norma.

\subsection{Organização do Trabalho}

No Capítulo (2), apresentaremos os conceitos básicos e alguns exemplos de sistemas Hamiltonianos ligados principalmente a problemas na mecânica celeste. Mostraremos também algumas propriedades de sistemas com coeficientes periódicos, matrizes simpléticas e Hamiltonianas, algumas propriedades variacionais de sistemas Hamiltonianos. 
No Capítulo (3), iremos descrever o problema dos 3 corpos restrito, mostrar as equações de movimento, definir as coordenadas sinódicas e fixas utilizadas para descrever alguns sistemas. Apresentaremos também a relação entre esse problema e o problema dos 3 corpos geral, esclarecendo por quê o estudo de uma forma mais simples do problema nos faz entender o problema mais geral.

No Capítulo (4), estudaremos a estabilidade vertical, estabilidade dos movimentos periódicos no problema de Sitnikov circular. Vamos utilizar as propriedades exibidas anteriormente e extrair características desses movimentos, e também mostrar alguns resultados numéricos. 


\section{Capítulo 2}

\section{Sistemas Hamiltonianos}

O formalismo Hamiltoniano é o estudo geométrico dos espaços de fase de sistemas mecânicos, portanto queremos estudar problemas mecânicos utilizando a geometria de suas soluções. Um sistema mecânico Hamiltoniano é dado por uma variedade de dimensão par (espaço de fase), uma estrutura simplética nesse espaço (a integral de Poincaré) e uma função definida nesse mesmo espaço (a função Hamiltoniana). Portanto, o formalismo Hamiltoniano é a estrutura matemática natural, para se desenvolver a teoria de sistemas mecânicos conservativos, pois para esse tipo de sistemas mecânicos temos todas as estruturas requeridas.

O tratamento Hamiltoniano simplifica uma série de problemas mecânicos que visto por outra teoria se torna um porblema intratável (por exemplo, o problema de atração de dois centros fixos). O ponto de vista Hamiltoniano tem ainda mais valor pelo fato de algumas teorias importantes terem sido formuladas utilizando-se desse formalismo, como a teoria de perturbações, a teoria ergódica, a mecânica estatística, a mecânica quântica, etc.

Neste capítulo abordaremos brevemente sobre algumas definições básicas de sistemas Hamiltonianos com exemplos, [MHO09] e sistemas de equações diferenciais com coeficientes periódicos, [YS75].

\subsection{Fundamentos e exemplos}

Um sistema Hamiltoniano é um sistema de $2 n$ equações diferenciais ordinárias da forma

$$
\dot{q}=H_{p}, \quad \dot{p}=-H_{q},
$$

ou, em componentes,

$$
\dot{q}_{i}=\frac{\partial H}{\partial p_{i}}(t, q, p), \quad \dot{p}_{i}=-\frac{\partial H}{\partial q_{i}}(t, q, p), \quad i=1, \ldots, n,
$$

em que $H=H(t, q, p)$, que será chamada de Hamiltoniana, é uma função $H: \mathcal{O} \rightarrow \mathbb{R}$ onde $\mathcal{O} \subset \mathbb{R} \times \mathbb{R}^{n} \times \mathbb{R}^{n}$ e $\mathcal{O}$ é aberto. Os vetores $q=\left(q_{1}, \ldots, q_{n}\right)$ e $p=\left(p_{1}, \ldots, p_{n}\right)$ são tradicionalmente chamados de vetores posição e momento, respectivamente, e $t$ é o tempo. As variáveis $q$ e $p$ são ditas variáveis conjugadas.

Na notação (1.1), (2.1) pode ser reescrito como

$$
\dot{z}=J \nabla H(t, z) .
$$

Um resultado básico de equações diferenciais é o teorema de existência e unicidade. O teorema diz que se $H$ é de classe $\mathcal{C}^{1}$, para cada $\left(t_{0}, z_{0}\right) \in \mathcal{O}$, há uma única solução $z=\phi\left(t, t_{0}, z_{0}\right)$ de $(2.3)$, definida para $t$ num intervalo aberto que contém $t_{0}$, e que satisfaz a condição inicial $\phi\left(t_{0}, t_{0}, z_{0}\right)=z_{0}$.

No caso especial em que $H$ independe de $t$, ou seja, $H: \mathcal{O} \rightarrow \mathbb{R}$, onde $\mathcal{O}$ é um aberto de $\mathbb{R}^{2 n}$, a equação diferencial (2.3) é autônoma e o sistema Hamiltoniano é dito conservativo. Neste caso vale 
a chamada propriedade de translação para o fluxo: $\phi\left(t-t_{0}, 0, z_{0}\right)=\phi\left(t, t_{0}, z_{0}\right)$. Neste caso, também, pode-se eliminar a dependência de $t_{0}$ na solução, podendo-se escrever a solução como $\phi\left(t, z_{0}\right)$, assim podemos dizer que as soluções são curvas parametrizadas em $\mathcal{O} \subset \mathbb{R}^{2 n}$, e $\mathcal{O}$ é chamado de espaço de fase.

Considere agora o caso autônomo de (2.3),

$$
\dot{z}=J \nabla H(z) .
$$

Uma integral primeira, ou simplesmente uma integral para (2.4) é uma função suave $F: \mathcal{O} \rightarrow \mathbb{R}$, que é constante ao longo das soluções de $(2.4)$, isto é, $F\left(\phi\left(t, z_{0}\right)\right)=F\left(z_{0}\right)=$ constante. Exemplos de integrais são a energia, o momento. As superfícies de níveis $F^{-1}(c) \subset \mathbb{R}^{2 n}, c$ uma constante, são exemplos de conjuntos invariantes, isto é, são conjuntos tais que, se as soluções começam neles, permanecem nesse conjunto. Em geral, os conjuntos de níveis são variedades de dimensão $2 n-1$.

Agora iremos dar alguns exemplos de sistemas Hamiltonianos ligados a problemas em mecânica celeste.

1. Considere $N$ pontos materiais movendo-se num sistema de referência Newtoniano, $\mathbb{R}^{3}$, em que a única força atuante, seja a força de atração entre os mesmos. Seja $q_{i}$ o vetor posição da $i$-ésima partícula com massa $m_{i}$; então pela segunda lei de Newton e pela lei da gravitação universal temos a seguinte equação do movimento para a $i$-ésima massa

$$
m_{i} \ddot{q}_{i}=\sum_{j=1}^{N} \frac{G m_{i} m_{j}\left(q_{i}-q_{j}\right)}{\left\|q_{j}-q_{i}\right\|^{3}}=\frac{\partial U}{\partial q_{i}}
$$

onde

$$
U=-\sum_{1 \leq i<j \leq N} \frac{G m_{i} m_{j}}{\left\|q_{i}-q_{j}\right\|}
$$

$G$ é a constante gravitacional universal e $U$ é o potencial. O sistema de equações diferenciais (2.5) define o problema dos $N$ corpos (formulação Newtoniana).

Seja $q=\left(q_{1}, \ldots, q_{N}\right) \in \mathbb{R}^{3 N}$. O sistema $(2.5)$ pode ser escrito em sua forma vetorial da seguinte maneira

$$
M \ddot{q}-\nabla U(q)=0,
$$

onde $M=\operatorname{diag}\left(m_{1}, m_{1}, m_{1}, \ldots, m_{N}, m_{N}, m_{N}\right)$; a formulação Hamiltoniana do problema é obtida introduzindo os vetores dos momentos lineares. Defina $p=\left(p_{1}, \ldots, p_{N}\right) \in \mathbb{R}^{3 N}$ por $p=M \dot{q}$, portanto $p_{i}=m_{i} \dot{q}_{i}$ é o momento da $i$-ésima partícula. As equações do movimento tornam-se então

$$
\dot{q}=H_{p}=M^{-1} p, \quad \dot{p}=-H_{q}=-U_{q}
$$

ou em componentes,

$$
\dot{q}_{i}=\frac{\partial H}{\partial p_{i}}=p_{i} / m_{i}, \quad \dot{p}_{i}=-\frac{\partial H}{\partial q_{i}}=-\frac{\partial U}{\partial q_{i}}=\sum_{i=1}^{N} \frac{G m_{i} m_{j}\left(q_{j}-q_{i}\right)}{\left\|q_{i}-q_{j}\right\|^{3}}
$$

onde o Hamiltoniano é

$$
H=\frac{1}{2} p^{T} M^{-1} p+U=\sum_{i=1}^{N} \frac{\left\|p_{i}\right\|^{2}}{2 m_{i}}+U .
$$


H é a energia total do sistema de partículas. Ela é uma integral do sistema, já que

$$
\frac{d H}{d t}=\frac{\partial H}{\partial q} \dot{q}+\frac{\partial H}{\partial p} \dot{p}=0 .
$$

Os vetores $q$ e $p$ são ditos de variáveis conjugadas.

O problema dos $N$ corpos é um sistema de $3 N(6 N)$ equações de segunda ordem (primeira ordem na formulação Hamiltoniana), portanto uma solução para o problema requer $6 N-1$ integrais independentes do tempo. Para $N>2$ ter tantas integrais globais é quase impossível. Entretanto, para $N>2$ existem 10 integrais para sistema.

Seja

$$
L=p_{1}+\ldots+p_{N}
$$

O momento linear total. De (2.8) segue que $\dot{L}=0$. Isso nos dar $\ddot{C}=0$, em que

$$
C=m_{1} q_{1}+\ldots+m_{N} q_{N}
$$

é o centro de massa do sistema, pois $\dot{C}=L$. Portanto o momento linear total é constante, e o centro de massa do sistema move com velocidade uniforme retilíneo. Se tomarmos uma sistema de coordenadas no qual o centro de massa é fixado na origem, as 6 integrais se reduzem a:

$$
\begin{aligned}
& \sum m_{i} q_{i}^{(1)}=0, \sum m_{i} q_{i}^{(2)}=0, \sum m_{i} q_{i}^{(3)}=0 \\
& \sum m_{i} \dot{q}_{i}^{(1)}=0, \sum m_{i} \dot{q}_{i}^{(2)}=0, \sum m_{i} \dot{q}_{i}^{(3)}=0 .
\end{aligned}
$$

Há, também, 3 integrais as quais mostram que o momento angular é constante em torno de qualquer eixo fixado no espaço. Se tomarmos os eixos como os eixos coordenados, essa integrais são:

$$
\sum m_{i}\left(q_{i}^{(2)} \dot{q}_{i}^{(3)}-q_{i}^{(3)} \dot{q}_{i}^{(2)}\right)=0, \sum m_{i}\left(q_{i}^{(3)} \dot{q}_{i}^{(1)}-q_{i}^{(1)} \dot{q}_{i}^{(3)}\right)=0, \sum m_{i}\left(q_{i}^{(1)} \dot{q}_{i}^{(2)}-q_{i}^{(2)} \dot{q}_{i}^{(1)}\right)=0
$$

E a outra integral que sobra é a energia total do sistema.

\section{Problema de Kepler}

Um caso especial do problema de 2 corpos é obtido quando um corpo de massa $M$ é suposto fixado na origem (por exemplo, o Sol). Neste caso a equação Newtoniana do movimento do outro corpo de massa $m$ é da forma

$$
m \ddot{q}=\frac{G M m q}{\|q\|^{3}}, \text { ou } \ddot{q}=\frac{\mu q}{\|q\|^{3}}=\nabla U(q),
$$

em que $q \in \mathbb{R}^{3}$ é o vetor posição do corpo de massa $m$ num sistema de coordenadas fixo, $\mu$ é a constante $G M$, e $U$ o potencial

$$
U=-\frac{\mu}{\|q\|}
$$

Se definirmos o momento $p=\dot{q} \in \mathbb{R}^{3}$, então a equação Newtoniana pode ser escrita em sua forma Hamiltoniana 


$$
\dot{q}=H_{p}=p, \dot{p}=-H_{q}=\frac{\mu q}{\|q\|^{3}},
$$

onde

$$
H=\frac{\|p\|^{2}}{2}-\frac{\mu}{\|q\|} \cdot
$$

H é chamada a Hamiltoniana do problema de Kepler. A formulação Newtoniana é um sistema de três equações de segunda ordem, enquanto as equações Hamiltonianas são seis equações de primeira ordem.

H é uma integral do movimento, isto é, é constante ao longo das soluções, pois

$$
\frac{d H}{d t}=0
$$

Definindo $A=q \times p$, como o momento angular, temos

$$
\dot{A}=\dot{q} \times p+q \times \dot{p}=p \times p+q \times\left(-\frac{\mu q}{\|q\|^{3}}\right)=0,
$$

portanto o momento angular $A$ é constante ao longo das soluções, logo as três componentes de $A$ são integrais. Se $A=0$, então

$$
\frac{d}{d t}\left(\frac{q}{\|q\|}\right)=\frac{(q \times \dot{q}) \times q}{\|q\|^{3}}=\frac{A \times q}{\|q\|^{3}}=0 .
$$

Portanto se o momento angular é nulo, o movimento é retilíneo. O uso dessa reta do movimento como um dos eixos de coordenadas, transforma-se o problema em um problema de um grau de liberdade, e portanto integrável. Neste caso as integrais são elementares e pode-se obter fórmulas simples para elas.

Se $A \neq 0$, então $q$ e $p=\dot{q}$ são ortogonais a $A$, portanto o movimento ocorre num plano ortogonal a $A$, plano invariante. Neste caso o problema é reduzido a um problema com dois graus de liberdade com uma integral, e tal problema é integrável.

\subsection{Sistemas de equações diferenciais lineares}

\subsubsection{Definições básicas}

Nesta seção estaremos preocupados com sistemas do tipo:

$$
\frac{d x_{i}}{d t}=a_{i 1}(t) x_{1}+\ldots+a_{i n}(t) x_{n}, \quad i=1, \ldots, n,
$$

em que a variável independente $t$ é real e os coeficientes $a_{i j}(t)(j, i=1, \ldots, n)$ são geralmente funções a valores complexos, contínuas por parte e integráveis em qualquer intervalo finito $\left(t_{1}, t_{2}\right)$. Em notação matricial, o sistema (2.14), torna-se

$$
\frac{d x}{d t}=A(t) x,
$$

em que $x=\left(x_{1}, \ldots, x_{n}\right)^{T}$ e $A(t)=\left[a_{i j}(t)\right]$ é a matriz $n \times n$ com coeficientes $a_{i j}(t)$.

Sejam $x^{i}(t)(i=1, \ldots, k) k$ soluções arbitrárias da equação $(2.15)$ ( $x^{i}(t)$ é um vetor coluna) com condições iniciais $x^{i}(0)=\alpha^{i}(i=1, \ldots, k)$. Então a matriz $n \times k$

$$
X(t)=\left[x_{1}(t) \ldots x_{k}(t)\right]
$$


satisfaz o seguinte sistema matricial

$$
\frac{d X}{d t}=A(t) X
$$

$\mathrm{e}$

$$
X(0)=\left[\alpha_{1} \ldots \alpha_{k}\right]
$$

Suponha $k=n$ e $\operatorname{det} X(0) \neq 0$. Qualquer outra solução $X_{1}(t), n \times n$, da equação (2.16) pode ser expressa como

$$
X_{1}(t)=X(t) C
$$

onde $C=X^{-1}(0) X_{1}(0)$.

Neste caso temos a fórmula de Liouville-Jacobi ou Teorema de Liouville

$$
\operatorname{det} X_{1}(t)=\operatorname{det} X_{1}(0) \exp \int_{0}^{t} \operatorname{Tr} A(t) d t .
$$

Definição. Um conjunto fundamental de soluções da equação (2.15) é qualquer conjunto de $n$ soluçôes $x^{1}(t), \ldots, x^{n}(t)$ que são linearmente independentes. A matriz $X(t)$ com colunas $x^{1}(t), \ldots, x^{n}(t)$, portanto, satisfaz (2.16), e é chamada de matriz fundamental. No caso especial em que $X(0)=I_{n}$ chamamos essa matriz de matriz principal.

Vamos interpretar agora (2.16) como um operador que dada uma matriz $A(t)$ devolve a matriz principal $X(t)$, isto é uma matriz $X(t)$ que satisfaça (2.16) e $X(0)=I$. Ou seja, vamos definir um conjunto $\Omega$ de todas as matrizes $A(t), n \times n$, contínuas por partes e integráveis em $[0, T], T>0$ e o conjunto $\Delta$ das matrizes $n \times n$ não-singulares e contínuas, definida num intervalo $[0, T], T>0$, com derivada contínua por partes, tal que $X(0)=I$ e $X(t)$ satisfazendo (2.16). Podemos considerar naturalmente

$$
\begin{gathered}
\Omega=\left\{A:[0, T] \rightarrow \mathcal{M}_{n \times n}(\mathbb{R}) ; A \text { continua por partes e integravel }\right\} \\
\Delta=\{X:[0, T] \rightarrow G L(n) ; X(0)=I, X \text { continua, com derivada continua por partes } \\
e \dot{X}=A X \text { para algum } A \in \Omega\},
\end{gathered}
$$

Defina uma norma em $\Omega$ e uma métrica em $\Delta$ por

$$
\|A\|=\int_{0}^{T}|A(t)| d t, \quad \rho\left(X_{1}, X_{2}\right)=\sup _{0 \leq t \leq T}\left|X_{1}(t)-X_{2}(t)\right|+\int_{0}^{T}\left|\dot{X}_{1}(t)-\dot{X}_{2}(t)\right| d t,
$$

onde $|Z|$ denota a norma da matriz $Z=\left|\nu_{i j}\right|$ :

$$
|Z|=\sqrt{\sum_{i, j}\left|\nu_{i j}\right|^{2}}
$$

Mostraremos que matrizes $A_{1}(t)$ e $A_{2}(t)$ "próximas"na norma ||.|| determinam matrizes principais $Z_{1}(t)$ e $Z_{2}(t)$ "próximas"na métrica $\rho$ e vice-versa, ou seja, vale:

Proposição ([YS75]) 1. $\Omega$ e $\Delta$ são homeomorfos, ou seja, existe uma aplicação bijetora contínua com inversa contínua que leva matrizes de $\Omega$ em matrizes de $\Delta$.

Demonstração. bijetividade:

A bijetividade do operador sai direto da definição de $\Omega$ e $\Delta$. A injetividade sai do teorema da unicidade de soluções, dado $A(t)$ pela unicidade das soluções existe um único $X(t) \in \Delta$ satisfazendo (2.16). A sobrejetividade sai do fato que dado $X(t) \in \Delta, X(t)$ é não singular e satisfaz (2.16) para alguma $A(t) \in \Omega$ portanto, dado $X(t) \in \Delta$ vale 


$$
A(t)=\frac{d X}{d t} X^{-1}(t)
$$

\section{Continuidade:}

Dado $X(t) \in \Delta, \exists \delta>0$ tal que para todo $X_{1}(t) \in \Delta \operatorname{com} \rho\left(X_{1}, X\right)<\delta$, temos que o $\sup _{0 \leq t \leq T}\left|X_{1}(t)-X(t)\right|<\delta$ como $X(t)$ e $X_{1}(t)$ são não singulares, ou seja, $\operatorname{det} X(t) \geq M>0$ $(0 \leq t \leq T) \forall X \in \Delta$ segue que $\exists \delta_{1}$ suficientemente pequeno tal que $\sup _{0 \leq t \leq T}\left|X_{1}^{-1}(t)-X^{-1}(t)\right|<\delta_{1}$

$$
\begin{gathered}
\left\|A_{1}-A\right\|=\int_{0}^{T}\left|A_{1}(t)-A(t)\right| d t=\int_{0}^{T}\left|\dot{X}\left(X^{-1}-X_{1}^{-1}\right)+\left(\dot{X}-\dot{X}_{1}\right) X_{1}^{-1}\right| d t \leq \\
\leq \sup _{0 \leq t \leq T}\left|X^{-1}-X_{1}^{-1}\right| \int_{0}^{T}|\dot{X}| d t+\sup _{0 \leq t \leq T}\left|X_{1}^{-1}\right| \int_{0}^{T}\left|\dot{X}-\dot{X}_{1}\right| d t .
\end{gathered}
$$

E o lado direito da desigualdade pode ficar tão pequeno quanto se queira para $\rho\left(X_{1}, X\right)$ suficientemente pequeno, completando a demonstração.

Segue do teorema que, se $\left\|A_{1}-A\right\|$ é suficientemente pequeno, então $\rho\left(X_{1}, X\right)$ também será, e, consequentemente o sup $\left|X_{1}-X\right|$, ou seja, para pequenas variações da matriz de coeficientes há uma pequena variação da matriz solução.

Vamos agora considerar o sistema adjunto de (2.15), em notação vetorial temos:

$$
\frac{d z}{d t}=-A^{*}(t) z
$$

em que $z=\left(z_{1}, \ldots, z_{n}\right)^{T}$ e $A^{*}(t)=\left|\bar{a}_{j i}(t)\right|$ é a matriz $n \times n$ com coeficientes $\bar{a}_{j i}(t)$. Se $A(t)$ é uma matriz real então temos $A^{*}(t)=A^{T}(t)$. Neste caso o sistema (2.20) é dito o associado com (2.15). Seja $Z(t)$ a matriz principal do sistema (2.20). Segue de (2.16) que

$$
\frac{d X^{*}}{d t}=X^{*} A^{*}(t)
$$

temos então que $X^{*}(t) Z(t)=I_{n}$. De fato, $X^{*}(0) Z(0)=I_{n}$, e derivando temos

$$
\frac{d}{d t}\left(X^{*} Z\right)=\frac{d X^{*}}{d t} Z+X^{*} \frac{d Z}{d t}=X^{*} A(t) Z-X^{*} A(t) Z=0 .
$$

Portanto as matrizes principais de (2.16) e (2.20) têm as seguintes relações

$$
Z(t)=\left[X^{*}(t)\right]^{-1} \text {. }
$$

Para quaisquer duas soluções $x(t)$ e $z(t)$ das equações (2.15) e (2.20), respectivamente, temos

$$
<x(t), z(t)>=\text { constante. }
$$

De fato,

$$
\frac{d}{d t}<x, z>=<\frac{d x}{d t}, z>+<x, \frac{d z}{d t}>=<A x, z>-<x, A^{*} z>=0 .
$$

É evidente de (2.22) que para qualquer solução $z(t)$ da equação $(2.20)$ a função $\psi(t, x)=(x, z(t))$ é uma integral da equação (2.15). A volta também vale.

\subsubsection{Sistemas de equações diferenciais lineares com coeficientes periódicos ar- bitrários}

Nesta seção admitiremos que para algum $T>0$ e todo $t$ temos

$$
A(t+T)=A(t)
$$

Em outras palavras, os coeficientes do sistema (2.15) são funções $T$-periódicas de $t$, em consequência sua matriz principal satisfaz a identidade

$$
X(t+T)=X(t) X(T) \quad(-\infty<t<\infty) .
$$


O resultado anterior vale devido à unicidade de soluções na equação (2.15). Mesmo que o sistema seja periódico $((2.15)$ com uma matriz da forma (2.23)), a matriz principal pode não ser periódica, e a matriz $X(T)$ é a medida de não periodicidade da matriz principal.

Definição. $X(T)$ é chamada de Matriz de Monodromia e seus autovalores de multiplicadores do sistema (2.15). O conjunto de todos os autovalores é chamado de espectro da equação (2.15).

Portanto, a matriz de monodromia é o valor que a matriz fundamental $X(t)$, definida pela condição inicial $X(0)=I_{n}$, assume em $t=T$, e os multiplicadores são as raízes da equação

$$
\operatorname{det}\left[X(T)-\lambda I_{n}\right]=0
$$

A equação anterior é chamada equação característica.

Agora daremos alguma informação sobre logaritmo de matriz. Aqui enunciaremos apenas um teorema quando a matriz é diagonal (caso seja de interesse olhar a seção (A)).

Uma matriz $R$ tem um logaritmo se existe uma matriz $Q$ tal que $R=e^{Q}$, e escrevemos $Q=\log R$. Em geral o logaritmo não é único, mesmo no caso real (por exemplo $I=e^{0}=e^{2 \pi J}$ ). Se $R$ tem um logaritmo, $R=e^{Q}$, então $R$ é não singular e tem uma raiz quadrada $R^{1 / 2}=e^{Q / 2}$.

Proposição ([YS75]) 2. Seja $R$ uma matriz não singular; então existe uma matriz $Q$ tal que $R=e^{Q}$. Se $R$ é real e tem raiz quadrada, então $Q$ pode ser tomada real.

Seja $\boldsymbol{K}$ a matriz constante definida por

$$
K=\frac{1}{T} \log X(T),
$$

onde $Y=\log X(T)$ é uma das soluções da equação $e^{Y}=X(T)$. Se $A(t)$ é real, então $X(T)$ é, também, uma matriz real. Em geral $K$ não é real (basta que um dos autovalores de $X(T)$ seja negativo, $K$ é complexa). Considere a função matricial

$$
F(t)=X(t) e^{-t K}
$$

Temos que, $F(t)=F(t+T)$. Portanto podemos escrever a matriz principal de (2.15) como

$$
X(t)=F(t) e^{t K},
$$

em que $F(t)$ é uma matriz $T$-periódica, contínua, não singular e com derivada integrável contínua por partes com $F(0)=I_{n}$.

No caso que $A(t)$ além de ser real, tiver todos os autovalores positivos, podemos supor como consequência $K$ e $F(t)$ são matrizes reais. O teorema de Floquet-Lyapunov, a sguir, versa sobre uma generalizaão do que está acima.

Proposição ([YS75]) 3. A matriz principal do sistema (2.15) com coeficientes T-periódicos pode ser expressada da forma (2.28), onde $F(t)$ é uma matriz $n \times n$, T-periódica, não singular para todo $t$, contínua, com derivada contínua por partes e integrável, e tal que $F(0)=I$, e $K$ é uma matriz constante. Reciprocamente, se $F(t)$ é uma matriz com as propriedades anteriormente enunciadas, e $K$ uma matriz qualquer constante, então a matriz $X(t)$ definida por (2.28) é a matriz principal de algum sistema (2.15) com coeficientes T-periódicos.

Definição. Considere um sistema não-autônomo:

$$
\dot{x}=f(x, t), \quad f: D \times \mathbb{R}^{+} \rightarrow \mathbb{R}^{n},
$$

em que $D \subset \mathbb{R}^{n}, f$ é localmente Lipschitz em $x$, contínua por partes em $t$ no conjunto $D \times \mathbb{R}^{+}$. $x=x_{0} \in D$ é chamado de ponto de equilíbrio da equação se

$$
f\left(x_{0}, t\right)=0 \forall t \geq t_{0}>0 .
$$


Definição. Considere o mesmo sistema não-autônomo da definição anterior, com $x\left(t_{0}\right)=x_{0}$, (problema de Cauchy) e solução $x(t)$. Dizemos que uma solução $\bar{x}=\bar{x}(t)$ desse problema é:

- Estável segundo Lyapunov: Se estiver definida em $\left[t_{0}, \infty[\right.$ e se, para todo $\epsilon>0$, existir $\delta=$ $\delta\left(\epsilon, t_{0}, x_{0}\right)>0$ tal que se $\left\|x_{0}^{1}-x_{0}\right\|<\delta$ então a solução $x(t)$ de $\bar{x}(t)=f(t, x), x\left(t_{0}\right)=x_{0}^{1}$, defini-se em $\left[t_{0}, \infty\left[e\|x(t)-\bar{x}(t)\|<\epsilon, \forall t \geq t_{0}\right.\right.$.

- Assintoticamente estável: Se for estável e valer

$$
\lim _{t \rightarrow \infty}\|x(t)-\bar{x}(t)\|=0 .
$$

- Instável: Se não for estável.

No caso do nosso sistema (2.15), $x_{0}=0$ é um equilíbrio $\left(\bar{x}(t)=x_{0}\right.$ é a solução trivial). Assim podemos afirmar que a solução trivial do sistema (2.15) é estável se e só se todas as soluções do sistema (2.15) são limitadas para $t \rightarrow \infty$, e assintoticamente estável se todas soluções tendem para 0 quando $t \rightarrow \infty$. Ou seja, vale o seguinte teorema

Proposição ([YS75]) 4. Seja $A(t)$ contínua em $\left[t_{0}, \infty\right], t_{0} \geq 0$. Então a solução trivial $x_{0}=0$ de (2.15) é Lyapunov estável se, e somente se, a matriz principal $X\left(t, t_{0}\right)$ for limitada.

De (2.28) podemos afirmar que as propriedades de estabilidade e instabilidade da solução trivial (isto é, se o limite das soluções quando $t \rightarrow \infty$ é finito ou infinito), ou razão de crescimento das soluções de (2.15) nas condições de (2.23) quando $t \rightarrow \infty$, depende exclusivamente da matriz $K$. Ou seja, se for limitado temos que o sistema é estável, caso $\lim _{t \rightarrow \infty} e^{t K} \rightarrow 0$ temos que o sistema é assintoticamente estável, e instável caso contrário. Por razões práticas, portanto, é suficiente caracterizar a matriz $K$ ou pelo menos seus autovalores.

Definição. Os autovalores da matriz $K$ são conhecidos como expoentes característicos do sistema (2.15).

Proposição ([YS75]) 5. Podemos expressar os expoentes característicos como

$$
\alpha_{k}=\frac{1}{T} \ln \lambda_{k}, \quad k=1, \ldots, n,
$$

onde $\alpha_{k}$ é o expoente característico associado ao multiplicador $\lambda_{k}$ do sistema.

Assim pela definição de expoente característico, a solução do sistema (2.15) é estável se, e somente se, seus expoentes característicos têm parte real não positiva, e é simples no caso do expoente nulo. Caso contrário, ou seja, caso exista pelo menos um expoente com parte real positiva ou um expoente nulo não simples, a solução é instável. A solução é assintoticamente estável se todos os expoentes característicos tiverem parte real estritamente negativa. Pelo teorema (5) podemos concluir os mesmos resultados usando as respectivas características dos multiplicadores. A tabela a seguir mostra os resultados em função dos expoentes e dos multiplicadores.

\subsection{Sistemas Hamiltonianos e Canônicos de Equações Diferenciais Lineares}

\subsubsection{Definições básicas}

Considere o Hamiltoniano de um sistema mecânico com $n$ graus de liberdade

$$
\mathcal{H}=\frac{1}{2} \sum_{i, j=1}^{2 n} h_{i j}(t) \xi_{i} \xi_{j} \quad\left(h_{j i}(t)=h_{i j}(t) ; i, j=1, \ldots, 2 n ; t_{0} \leq t \leq \infty\right),
$$




\begin{tabular}{|c|c|c|}
\hline $\begin{array}{l}\text { Propriedade da solu- } \\
\text { ção }\end{array}$ & Expoente característico & Multiplicadores \\
\hline $\begin{array}{l}\text { Estabilidade da solução } \\
\text { trivial, todas as soluções } \\
\text { são limitadas em }[0, \infty]\end{array}$ & $\begin{array}{l}\text { Parte real não negativa: expoente } \\
\text { característico puro imaginário ou } \\
\text { nulo correspondem a autovalores } \\
\text { simples }\end{array}$ & $\begin{array}{l}\text { Pertencem a bola unitária fe- } \\
\text { chada: no caso em que seu mó- } \\
\text { dulo é unitário correspondem a } \\
\text { autovalores simples }\end{array}$ \\
\hline $\begin{array}{l}\text { Estabilidade assintótica } \\
\text { da solução trivial }\end{array}$ & Parte real negativa & Pertencem a bola unitária aberta \\
\hline $\begin{array}{l}\text { Instabilidade da solução } \\
\text { trivial }\end{array}$ & $\begin{array}{l}\text { Pelo menos um expoente com } \\
\text { parte real positiva, ou expoente } \\
\text { puro imaginário ou nulo corres- } \\
\text { pondendo a autovalores não sim- } \\
\text { ples }\end{array}$ & $\begin{array}{l}\text { Pelo menos um dos multiplicado- } \\
\text { res fora da bola unitária, ou se } \\
\text { tiver valor unitário corresponde a } \\
\text { autovalores não simples }\end{array}$ \\
\hline $\begin{array}{l}\text { Existência de solução T- } \\
\text { periódica }\end{array}$ & $\begin{array}{l}\text { Para pelo menos um expo- } \\
\text { ente característico } \alpha_{k}, \alpha_{k} T= \\
(2 m \pi i), m \in \mathbb{Z}\end{array}$ & $\begin{array}{l}\text { Pelo menos um multiplicador } \\
\text { igual a } 1\end{array}$ \\
\hline $\begin{array}{l}\text { Existência de solu- } \\
\text { ção anti-periódica, } \\
x(t+T)=-x(t)\end{array}$ & $\begin{array}{l}\text { Para pelo menos um expoente ca- } \\
\text { racterístico } \alpha_{k}, \alpha_{k} T=(2 m+ \\
\text { 1) } \pi i, m \in \mathbb{Z}\end{array}$ & $\begin{array}{l}\text { Pelo menos um multiplicador } \\
\text { igual a }-1\end{array}$ \\
\hline
\end{tabular}

Tabela 2.1: características das soluções de acordo com o expoente ou multiplicadores do sistema (2.15)

isto é, uma forma quadrática nas coordenadas generalizadas $q_{k}=\xi_{k}$ e momentos generalizados $p_{k}=\xi_{k+n},(k=1, \ldots, n)$. As equações Hamiltonianas ficam da forma

$$
\frac{d q_{k}}{d t}=\frac{d \xi_{k}}{d t}=\frac{\partial \mathcal{H}}{\partial \xi_{k+n}}=\frac{\partial \mathcal{H}}{\partial p_{k}}, \quad \frac{d p_{k}}{d t}=\frac{d \xi_{k+n}}{d t}=-\frac{\partial \mathcal{H}}{\partial \xi_{k}}=-\frac{\partial \mathcal{H}}{\partial q_{k}},(k=1, \ldots, n)
$$

Reescrevendo o sistema (2.31) na forma matricial temos

$$
-J \frac{d x}{d t}=H(t) x
$$

onde $x=\left(\xi_{1}, \ldots, \xi_{2 n}\right)$ e $H(t)=\left[h_{i j}(t)\right]$.

Definição. Uma equação da forma

$$
\tilde{J} \frac{d z}{d t}=\tilde{H}(t) z
$$

onde $\tilde{H}(t) e ́$ uma matriz real simétrica e $\tilde{J}$ uma matriz constante não singular e anti simétrica, será chamada de equação canônica.

Consideremos, agora, matrizes com coeficientes complexos.

Definição. Toda equação da forma (2.33), com matrizes complexas $\tilde{J}$ e $\tilde{H}(t)$, agora $\tilde{J}$ com a propriedade de ser anti hermitiana, isto é, $\tilde{J}^{*}=-\tilde{J}$, det $\tilde{J} \neq 0$, e $\tilde{H}(t)$ hermitiana, será chamada de equação hamiltoniana.

Uma equação canônica é um caso particular de equação hamiltoniana. A matriz $\tilde{H}(t)$, nos dois casos, será chamada de Hamiltoniana.

Consideremos uma matriz Hamiltoniana real e constante, ou seja, $\tilde{H}(t)=H_{0}=$ constante. Dessa forma a equação característica para a equação (2.32) neste caso é

$$
\operatorname{det}\left(J H_{0}-\lambda I_{2 n}\right)=0
$$

Proposição ([YS75]) 6. Se $\lambda$ é um raiz da equação (2.34) então - $\lambda$ também é. 
Demonstração. $\operatorname{det}\left(J H_{0}-\lambda I_{2 n}\right)=\operatorname{det}\left(J H_{0}-\lambda I_{2 n}\right)^{T}=\operatorname{det}\left(-H_{0} J-\lambda I_{2 n}\right)=$

$$
=\operatorname{det}\left[J\left(-H_{0} J-\lambda I_{2 n}\right) J^{-1}\right]=\operatorname{det}\left(J H_{0}+\lambda I_{2 n}\right) \operatorname{det}\left(-I_{2 n}\right)=\operatorname{det}\left(J H_{0}+\lambda I_{2 n}\right) \text {. }
$$

Como consequência do teorema anterior temos que o conjunto das raízes do polinômio característico de $H_{0}$ é simétrico com relação aos eixos real e imaginário.

Corolário 1. Se todas as raízes do polinômio característico de $H_{0}$ são imaginários puros e são simples, então todas as soluçôes de (2.32) são limitadas na reta. Em particular elas não podem ser assintoticamente estáveis.

\subsubsection{Propriedades de soluções das equações canônicas e Hamiltonianas}

Proposição ([YS75]) 7. A matriz principal de uma equação Hamiltoniana satisfaz a eguinte identidade

$$
Z^{*}(t) \tilde{J} Z(t)=\tilde{J} .
$$

Se a matriz $Z(t)$ satisfaz a condição (2.35), é contínua, tem derivada integrável contínua por partes, e $Z(0)=I_{2 n}$, então vale a recíproca, ou seja, $Z(t)$ é a matriz principal de alguma equação Hamiltoniana.

Definição. Uma matriz real Z tal que

$$
Z^{T} \tilde{J} Z=\tilde{J}
$$

onde $\tilde{J}$ é uma matriz real, não singular, anti-simétrica, é denominada matriz Simplética (ou $\tilde{J}$-ortogonal).

Portanto, a matriz principal de uma equação canônica é uma matriz simplética para todo $t$. Disto resultam algumas propriedades da equação.

Propriedades da equação Hamiltoniana.

1. Para quaisquer duas soluções $z_{1}(t), z_{2}(t)$ de uma equação Hamiltoniana, temos

$$
<\tilde{J} z_{1}(t), z_{2}(t)>\equiv \text { constante }
$$

Demonstração. $<\tilde{J} z_{1}(t), z_{2}(t)>=<\tilde{J} Z(t) z_{1}(0), Z(t) z_{2}(0)>=<Z^{*}(t) \tilde{J} Z(t) z_{1}(0), z_{2}(0)>=$ $=<\tilde{J} z_{1}(0), z_{2}(0)>$.

2. Suponha que a equação Hamiltoniana tenha uma integral primeira linear, isto é, uma integral da forma $\psi(t, z)=(z, v(t))$. Então a função $w(t)=\tilde{J}^{-1} v(t)$ é uma solução da equação (2.33).

Demonstração. Usando a propriedade do sistema adjunto (ver (2.20)) v(t) é uma solução da equação adjunta

$$
\frac{d v}{d t}=-\left(\tilde{J}^{-1} \tilde{H}(t)\right)^{*} v=\tilde{H}(t) \tilde{J}^{-1} v .
$$

Como $v=\tilde{J} w$, temos, $\tilde{J} \frac{d w}{d t}=\tilde{H}(t) w$.

No caso em que $\tilde{J}=-J$, isso nos diz que $-J^{-1} v=J v$, isto é, o vetor com componentes $\vartheta_{n+1}, \ldots, \vartheta_{2 n},-\vartheta_{1}, \ldots,-\vartheta_{n},\left(v=\left(\vartheta_{1}, \ldots, \vartheta_{2 n}\right)\right)$ é solução de $(2.32)$.

O lado esquerdo de (2.36) para duas soluções reais $x_{1}$ e $x_{2}$ da equação (2.32) é denominado por Invariante de Poincaré; quando $\tilde{J}=-J,(2.36)$ torna-se

$$
\sum_{k=1}^{n}\left(\xi_{k}^{1} \xi_{k+n}^{2}-\xi_{k+n}^{1} \xi_{k}^{2}\right)=\text { constante }
$$

onde $\xi_{1}^{i}, \ldots, \xi_{2 n}^{i}$ são as componentes de do vetor $x_{i}$. 


\subsubsection{Teorema de Lyapunov-Poincaré}

A partir de agora admitiremos que a matriz Hamiltoniana terá como coeficientes funções, além de contínuas por partes e integráveis, também T-periódicas.

$$
\tilde{J} \frac{d x}{d t}=\tilde{H}(t) x \quad\left(\tilde{J}^{T}=-\tilde{J}, \tilde{H}(t+T)=\tilde{H}(t)=\tilde{H}(t)^{T}, 0 \leq t \leq T\right),
$$

em que $\tilde{H}(t)$ é uma matriz real, simétrica e T-periódica.

Segue de (2.35) que

$$
X(T)=\tilde{J}^{-1}\left[X(T)^{T}\right]^{-1} \tilde{J},
$$

em que $X(T)$ é a matriz de monodromia (valor da matriz principal ao final de um período $\mathrm{T}$ ) da equação (2.38).

Seja $\left\{\lambda_{i}\right\}$ um conjunto de multiplicadores da equação (2.38). As matrizes $X(T)$ e $X(T)^{T}$ têm os mesmos autovalores. Portanto os autovalores da matriz $\left[X(T)^{T}\right]^{-1}$ são os valores $\lambda_{i}$. Matrizes similares têm os mesmos autovalores, logo, segue de (2.39) que os conjuntos $\left\{\lambda_{i}\right\}$ e $\left\{\lambda_{i}^{-1}\right\}$ são idênticos. Isso prova o seguinte teorema:

Proposição ([YS75]) 8. A equação característica da equação canônica (2.38)

$$
\operatorname{det}\left[X(T)-\lambda I_{2 n}\right]=\lambda^{2 n}+a_{1} \lambda^{2 n-1}+\ldots+a_{2 n-1} \lambda+a_{2 n}=0, \quad a_{i} \in \mathbb{R}
$$

é reversivel, isto é, $a_{2 n}=1 ; a_{2 n-k}=a_{k}, k=1, \ldots, n$.

No caso de coeficientes complexos e uma matriz T-periódica e hermitiana $\tilde{H}(t)$, a equação canônica passa a ser uma equação Hamiltoniana e pode-se trocar o $\tilde{H}(t)^{T}$ por $\tilde{H}(t)^{*}$ e $Z(T)^{T}$ por $Z(T)^{*}$ e segue o raciocínio anterior.

O conjunto dos autovalores de $Z(T)^{*}$ é $\left\{\bar{\lambda}_{i}\right\}$. Logo, segue que os conjuntos $\left\{\bar{\lambda}_{i}^{-1}\right\}$ e $\left\{\lambda_{i}\right\}$ coincidem. Assim, o espectro de uma equação Hamiltoniana é simétrico em relação ao círculo unitário. No caso de uma equação canônica, o espectro também é simétrico em relação ao eixo real, logo se $\lambda$ for um multiplicador, fora do círculo unitário, temos que $\bar{\lambda}^{-1}, \bar{\lambda}$ e $\lambda^{-1}$ também são multiplicadores (isso segue do fato a equação característica de uma equação canônica ter coeficientes reais). Portanto temos o teorema de Lyapunov-Poincaré:

Proposição ([YS75]) 9. Os multiplicadores de uma equação Hamiltoniana (canônica), contando sua multiplicidades, são simétricos (no sentido de inversão) em relação ao círculo unitário.

O teorema de Lyapunov-Poincaré implica a seguinte proposição:

Proposição ([YS75]) 10. A solução trivial de um sistema canônico (sistema Hamiltoniano) é estável se e somente se todos os seus multiplicadores (isto é raízes da equação característica) estão no bordo do círculo unitário e são simples.

Teorema 1. Suponha que uma equação Hamiltoniana com matriz Hamiltoniana $\tilde{H}_{0}(t)$ tem multiplicadores simples, todos no bordo do círculo unitário. Então existe $\epsilon>0$ tal que a solução trivial de qualquer equação do tipo (2.38) com matriz Hamiltoniana $\tilde{H}(t)$ tal que

$$
\left\|\tilde{H}-\tilde{H}_{0}\right\|=\int_{0}^{T}\left|\tilde{H}(t)-\tilde{H}_{0}(t)\right| d t<\epsilon,
$$

é estável.

É assumido aqui que a perturbação aplicada na matriz $\tilde{H}_{0}(t)$ que a muda para uma matriz $\tilde{H}(t)$, seja uma perturbação que preserve a forma da equação Hamiltoniana (2.38), ou seja, a matriz $\tilde{H}(t)$ é hermitiana (ou real simétrica) com elementos T-periódicos. Caso a perturbação dada deixe a matriz resultante $\tilde{H}(t)$ não Hamiltoniana o teorema passa a ser falso. O teorema vale, em particular, para $\tilde{H}_{0}(t)=\tilde{H}_{0}=$ constante. 
Colocando em termos do espectro da matriz de monodromia $X(T)$ do sistema, se perturbarmos a matriz $\tilde{H}_{0}(t)$ continuamente preservando sua forma, a matriz de monodromia e seus autovalores, também são perturbados continuamente, preservando sua simetria em relação ao círculo unitário. Isso implica que os multiplicadores se movem no plano complexo de forma especial.

Se todos os multiplicadores do sistema (2.38) estão no bordo do círculo unitário, e a perturbação produz um multiplicador não real de valor absoluto $\neq 1$, então necessariamente aparecem quatro multiplicadores $\lambda, \bar{\lambda}, \lambda^{-1}$ e $\bar{\lambda}^{-1}$; no caso de aparecer um multiplicador real $\lambda \neq \pm 1$, teremos um par de multiplicadores $\lambda$ e $\lambda^{-1}$ novos. E isso significa que esses multiplicadores novos a aparecer serão associados à existência de multiplicadores não simples sobre o círculo unitário do sistema não perturbado.

\subsubsection{Equações variacionais de Poincaré e suas propriedades}

Considere um sistema mecânico holônomo. Denote as coordenadas e o momento como $q_{k}$ e $p_{k}(k=1, \ldots, n)$ respectivamente e o Hamiltoniano por $\mathcal{H}\left(q_{1}, \ldots, q_{n}, p_{1}, \ldots, p_{n}\right)$. Nosso ponto de partida será a equação de movimento em sua forma canônica

$$
\frac{d q_{k}}{d t}=\frac{\partial \mathcal{H}}{\partial p_{k}}, \frac{d p_{k}}{d t}=-\frac{\partial \mathcal{H}}{\partial q_{k}}, k=1, \ldots, n .
$$

Suponha que o sistema anterior tem uma solução periódica

$$
q_{k}=q_{k}^{(0)}(t), p_{k}=p_{k}^{(0)}(t) k=1, \ldots, n,
$$

e, em alguma vizinhança dessa solução, $\mathcal{H}$ é analítica. Os valores das coordenadas e momentos para um movimento perturbado

$$
q_{k}=q_{k}^{(0)}(t)+\xi_{k}, p_{k}=p_{k}^{(0)}(t)+\xi_{k+n} k=1, \ldots, n,
$$

satisfaz o mesmo sistema de equações diferenciais (2.40). Substituindo (2.42) em (2.40) e expandindo em série de taylor com respeito a perturbação $\xi_{k}(k=1, \ldots, 2 n)$ obtemos um sitema de equações diferenciais para o movimento perturbado:

$$
\begin{aligned}
\frac{d \xi_{k}}{d t} & =\sum_{i=1}^{n}\left[\left(\frac{\partial^{2} \mathcal{H}}{\partial p_{k} \partial q_{i}}\right)_{0} \xi_{i}+\left(\frac{\partial^{2} \mathcal{H}}{\partial p_{k} \partial p_{i}}\right)_{0} \xi_{i+n}\right]+E_{k} \\
\frac{d \xi_{k+n}}{d t} & =-\sum_{i=1}^{n}\left[\left(\frac{\partial^{2} \mathcal{H}}{\partial q_{k} \partial q_{i}}\right)_{0} \xi_{i}+\left(\frac{\partial^{2} \mathcal{H}}{\partial q_{k} \partial p_{i}}\right)_{0} \xi_{i+n}\right]+E_{k+n}, k=1,2, \ldots, n .
\end{aligned}
$$

Aqui $E_{k}, k=1, \ldots, 2 n$, contem os termos de ordem superior ou igual a dois da perturbação. Se neglegenciarmos esses termos, obtemos o que, chamamos de Equações Variacional de Poincaré. Estas equações formam um sistema de equações diferenciais linear com coeficientes periódicos do tipo (2.31).

Portanto podemos expressar (2.43) na forma matricial, usando como $H(t)$ a seguinte matriz:

$$
H(t)=\left(\begin{array}{cccccc}
\left(\frac{\partial^{2} H}{\partial q_{1}^{2}}\right)_{0} & \cdots & \left(\frac{\partial^{2} H}{\partial q_{n} \partial q_{1}}\right)_{0} & \left(\frac{\partial^{2} H}{\partial p_{1} \partial q_{1}}\right)_{0} & \cdots & \left(\frac{\partial^{2} H}{\partial p_{n} \partial q_{1}}\right)_{0} \\
\cdots & \cdots & \cdots & \cdots & \cdots & \cdots \\
\left(\frac{\partial^{2} H}{\partial q_{1} \partial q_{n}}\right)_{0} & \cdots & \left(\frac{\partial^{2} H}{\partial q_{n}^{2}}\right)_{0} & \left(\frac{\partial^{2} H}{\partial p_{1} \partial q_{n}}\right)_{0} & \cdots & \left(\frac{\partial^{2} H}{\partial p_{n} \partial q_{n}}\right)_{0} \\
\left(\frac{\partial^{2} H}{\partial q_{1} \partial p_{1}}\right)_{0} & \cdots & \left(\frac{\partial^{2} H}{\partial q_{n} \partial p_{1}}\right)_{0} & \left(\frac{\partial^{2} H}{\partial p_{1}^{2}}\right)_{0} & \cdots & \left(\frac{\partial^{2} H}{\partial p_{n} \partial p_{1}}\right)_{0} \\
\cdots & \cdots & \cdots & \cdots & \cdots & \cdots \\
\left(\frac{\partial^{2} H}{\partial q_{1} \partial p_{n}}\right)_{0} & \cdots & \left(\frac{\partial^{2} H}{\partial q_{n} \partial p_{n}}\right)_{0} & \left(\frac{\partial^{2} H}{\partial p_{1} \partial p_{n}}\right)_{0} & \cdots & \left(\frac{\partial^{2} H}{\partial p_{n}^{2}}\right)_{0}
\end{array}\right)
$$

onde o zero subscrito quer dizer que as derivadas parciais são calculadas na solução (2.41). Algumas 
das propriedades, além das que já foram enunciada para (2.33), são:

1. A equação (2.43) possui uma solução periódica particular,

$$
\xi_{k}=\dot{q}_{k}^{(0)}(t), \xi_{k+n}=\dot{p}_{k}^{(0)}(t), \quad k=1, \ldots, n
$$

2. Seja $\phi\left(t, q_{1}, \ldots, q_{n}, p_{1}, \ldots, p_{n}\right)=a$, uma integral primeira do sistema canônico original (2.40), analítica na vizinhança da solução (2.41). Então sua variação,

$$
\delta \phi=\sum_{i=1}^{n}\left[\left(\frac{\partial \phi}{\partial q_{i}}\right)_{0} \xi_{i}+\left(\frac{\partial \phi}{\partial p_{i}}\right)_{0} \xi_{i+n}\right]
$$

é uma integral primeira da equação variacional. 


\section{Capítulo 3}

\section{Descrição do problema restrito}

Neste capítulo introduziremos a noção de problema restrito dos três ou mais corpos. Como há várias maneiras de se definir o "problema restrito", precisamos ser mais precisos. Em termos gerais um problema restrito significa que uma ou mais partículas têm massas iguais a zero (chamadas de infinitesimais ou satélites) e várias outras partículas têm massas finitas (chamadas de primárias). Podemos supor que as $k$ primárias descrevem alguma solução conhecida do problema dos $k$ corpos, enquanto que as infinitesimais estão sob influência gravitacional das primárias. Porém como as infinitesimais "não"têm massa, elas não influenciam o movimento das primárias ou os movimentos umas das outras.

O que chamaremos de problema restrito é, mais propriamente, chamado de problema restrito circular dos 3 corpos. As três partículas têm massas $\mu_{1}, \mu_{2}, 0$. As primárias de massas $\mu_{1}$ e $\mu_{2}$, (neste caso $k=2$ ) movem-se em uma solução circular do problema dos 2 corpos e portanto o nome, "circular". Como esse caso foi o mais estudado, principalmente por Poincaré e Birkhoff o adjetivo circular será deixado de lado e esse será chamado de "o"problema restrito.

Neste capítulo detalharemos o problema restrito no plano e no espaço, [Sze67] e introduziremos o problema de configurações centrais para, assim, abordar o problema restrito dos $(N+1)$ corpos, [Mey99].

\subsection{Introdução}

Szebehely em [Sze67], cita que na física, em geral, a maior dificuldade em atacar um problema é a falta ou a escassez de definições claras, uma vez resolvido esse problema, podemos assim "atacar"o problema. Um contra exemplo de que nem sempre boas definições do problema nos leva a resolvê-lo é o problema dos três corpos, que tem uma formulação clara, mas é um problema difícil de ser atacado em sua forma geral. Pensando nisso, tentaremos descrever o problema restrito de maneira mais clara possível, para um bom entendimento do mesmo.

Após definir o problema, mostraremos as equações do movimento em coordenadas inerciais (sideral) e rotacionais (sinódica). Para isso usaremos tanto variáveis físicas ou dimensionais (variáveis com algum sentido físico, ou seja, com unidades de medida) como as não-físicas ou adimensionais (sem unidades de medidas). No final as quatros equações serão comparadas, e mostraremos como, ao introduzirmos as coordenadas sinódicas, resulta a existência da constante e da integral Jacobiana.

\subsection{Descrição do problema planar}

\subsubsection{Definição do problema e as equações do movimento no sistema inercial}

Definiremos nosso problema como: dois corpos (primárias) girando em torno de seu centro de massa, em órbitas circulares sob influência da atração gravitacional mútua, e um terceiro corpo (corpo infinitesimal, atraído pelos outros dois corpos, mas que não influencia o movimento deles) 
move-se no plano definido pelas primárias. O problema restrito dos 3 corpos é descrever o movimento do terceiro corpo.

Suponha que as primárias tenham massas arbitrárias $m_{1}$ e $m_{2}$, que, devido à distribuição dessas massas, podemos considerar as primárias como pontos materiais (ao invés de pensarmos em corpos rígidos). Deixaremos para depois uma explicação detalhada da terceira massa, $m_{3}$, por ser um definição complexa, mas até este ponto podemos considerar $m_{3}$ muito menor que $m_{2}$ e $m_{1}$, pois supõe-se que ela não influencia o movimento das outras duas.

Vamos supor que o centro de massa de $m_{1}$ e $m_{2}$ esteja na origem, 0 , ver figura (3.1). Usando esse fato queremos que o balanceamento entre a força centrífuga e a gravitacional seja:

$$
k^{2} \frac{m_{1} m_{2}}{l^{2}}=m_{2} a n^{2}=m_{1} b n^{2},
$$

onde $k$ é a constante gravitacional Gaussiana, $n$ é a velocidade angular (igual para as duas massas) de $m_{1}$ e $m_{2}, l$ é a distância entre as primárias e $a$ e $b$ são as ditâncias de $m_{2}$ ao centro de massa e de $m_{1}$ ao centro de massa, respectivamente. A quantidade $n$, em mecânica celeste, é chamada de movimento médio e o ângulo $n t^{*}$ a longitude de $m_{1}$. O símbolo $t^{*}$ é usado para o tempo dimensional, iremos preservar a notação $t$ para o tempo adimensional, ou seja, um número real qualquer.

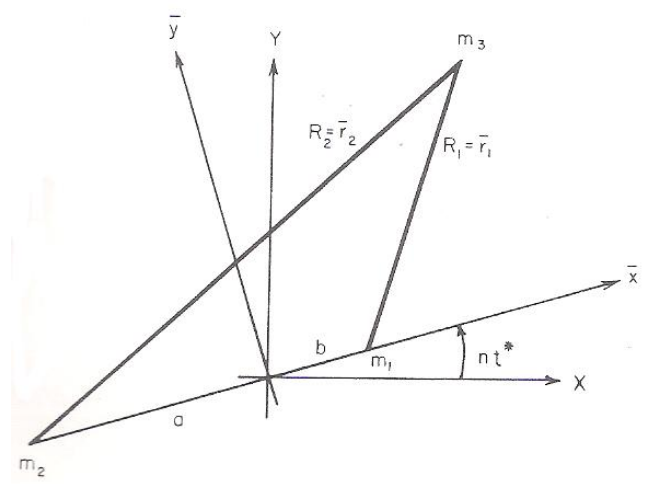

Figura 3.1: Configuração em coordenadas inerciais e rotacionais $\left(m_{1}>m_{2}\right)$.

Disso temos que

$$
k^{2} m_{1}=a n^{2} l^{2}, k^{2} m_{2}=b n^{2} l^{2}, k^{2}\left(m_{1}+m_{2}\right)=n^{2} l^{3},
$$

e a última equação é a terceira lei de Kepler.

Também temos

$$
a=\frac{m_{1} l}{M}, \quad b=\frac{m_{2} l}{M},
$$

onde $M=m_{2}+m_{1}$.

As equações de movimento de $m_{3}$ em um sistema de coordenadas retangular (cartesiano, $X Y$, ver figura 3.1) inercial, são

$$
\frac{d^{2} X}{d t^{* 2}}=\frac{\partial F}{\partial X}, \quad \frac{d^{2} Y}{d t^{* 2}}=\frac{\partial F}{\partial Y} .
$$

O sistema de coordenadas inercial $X, Y$ mostrado na figura (3.1) é chamado de sistema sideral. Acima, $F$ é a função potencial ou o potencial negativo ( $-F$ é a energia potencial) e é dado por

$$
F=k^{2}\left(m_{1} / R_{1}+m_{2} / R_{2}\right) .
$$


As distâncias $R_{1}$ e $R_{2}$ dadas por

$$
R_{i}=\left[\left(X-X_{i}\right)^{2}+\left(Y-Y_{i}\right)^{2}\right]^{1 / 2}, i=1,2,
$$

onde $\left(X_{1}, Y_{1}\right)$ e $\left(X_{2}, Y_{2}\right)$ são as coordenadas de $m_{1}$ e $m_{2}$, respectivamente, são obtidas olhando a figura (3.1):

$$
\begin{gathered}
X_{1}=b \cos \left(n t^{*}\right), X_{2}=-a \cos \left(n t^{*}\right) \\
Y_{1}=b \sin \left(n t^{*}\right), Y_{2}=-a \sin \left(n t^{*}\right) .
\end{gathered}
$$

A dependência no tempo das coordenadas das primárias introduz o tempo explicitamente nas equações de movimento de $m_{3}$. Isso é esperado, já que $m_{1}$ e $m_{2}$ movem-se em um sistema de coordenadas fixado.

Substituindo (3.7), (3.5) e (3.6) em (3.4) vem que:

$$
\begin{aligned}
& \frac{d^{2} X}{d t^{* 2}}=-k^{2}\left[\frac{m_{1}\left(X-b \cos \left(n t^{*}\right)\right)}{R_{1}^{3}}+\frac{m_{2}\left(X+a \cos \left(n t^{*}\right)\right)}{R_{2}^{3}}\right], \\
& \frac{d^{2} Y}{d t^{* 2}}=-k^{2}\left[\frac{m_{1}\left(Y-b \sin \left(n t^{*}\right)\right)}{R_{1}^{3}}+\frac{m_{2}\left(Y+a \sin \left(n t^{*}\right)\right)}{R_{2}^{3}}\right] .
\end{aligned}
$$

\subsubsection{Equações do movimento no sistema de coordenadas sinódicas e a integral Jacobiana}

A função potencial $F$ depende do tempo explicitamente, por causa do movimento das primárias. Consequentemente o Hamiltoniano depende explicitamente do tempo, portanto, ele deixa de ser uma integral primeira, ou seja, não é constante ao longo de soluções. Baseado nisso, surgiu a pergunta: Qual sistema de coordenadas poderia resultar uma expressão da força que não dependa explicitamente do tempo? A resposta intuitiva é a seguinte: como a dependência do tempo é uma consequência do movimento das primárias nas coordenadas fixadas, se passarmos a usar um sistema de coordenadas em que as mesmas fiquem fixas, talvez neste sistema de coordenadas a função força passe a ser autônoma.

Para isso vamos considerar um referencial móvel cujo o eixo $O \tilde{x}$ é paralelo a reta que liga as primárias e gira em torno do eixo $O \tilde{z}$.

Essa transformação de coordenadas é mais conhecida como rotação, e usando a notação da figura (3.1) teremos:

$$
\begin{array}{r}
X=\tilde{x} \cos \left(n t^{*}\right)-\tilde{y} \sin \left(n t^{*}\right), \\
Y=\tilde{x} \sin \left(n t^{*}\right)+\tilde{y} \cos \left(n t^{*}\right),
\end{array}
$$

ou, em notação matricial,

$$
R=A \tilde{r},
$$

onde o vetor $R=(X, Y), \tilde{r}=(\tilde{x}, \tilde{y})$ e $A$ é a matriz dada por

$$
A=\left(\begin{array}{cc}
\cos \left(n t^{*}\right) & -\sin \left(n t^{*}\right) \\
\sin \left(n t^{*}\right) & \cos \left(n t^{*}\right)
\end{array}\right) .
$$

A transformação aplicada às equações (3.9) fica mais simples se introduzirmos variáveis complexas. Seja

$$
Z=z e^{i n t^{*}},
$$

onde

$$
z=\tilde{x}+i \tilde{y}, Z=X+i Y .
$$


As distâncias $R_{1}$ e $R_{2}$ são dadas por

$$
R_{i}=\left|Z-Z_{i}\right|, \quad i=1,2,
$$

onde, de acordo com a equação (3.9)

$$
Z_{1}=b e^{i n t^{*}} \text { e } Z_{2}=-a e^{i n t^{*}} .
$$

Portanto, usando (3.14) e (3.16), (3.15) fica da seguinte forma

$$
\begin{aligned}
& R_{1}=|z-b|=\left[(\tilde{x}-b)^{2}+\tilde{y}^{2}\right]^{1 / 2}, \\
& R_{2}=|z+a|=\left[(\tilde{x}+a)^{2}+\tilde{y}^{2}\right]^{1 / 2} .
\end{aligned}
$$

O lado esquerdo de (3.9) usando notação complexa fica da forma

$$
\frac{d^{2} Z}{d t^{* 2}}=\left(\frac{d^{2} z}{d t^{* 2}}+2 i n \frac{d z}{d t^{*}}-n^{2} z\right) e^{i n t^{*}} .
$$

A forma complexa das equações de movimento no sistema em rotação é

$$
\frac{d^{2} z}{d t^{* 2}}+2 i n \frac{d z}{d t^{*}}-n^{2} z=-k^{2}\left[m_{1} \frac{(z-b)}{|z-b|^{3}}+m_{2} \frac{z+a}{|z+a|^{3}}\right],
$$

e as partes real e imaginária ficam

$$
\begin{aligned}
\frac{d^{2} \tilde{x}}{d t^{* 2}}-2 n \frac{d \tilde{y}}{d t^{*}}-n^{2} \tilde{x} & =-k^{2}\left[m_{1} \frac{(\tilde{x}-b)}{\tilde{r}_{1}^{3}}+m_{2} \frac{\tilde{x}+a}{\tilde{r}_{2}^{3}}\right], \\
\frac{d^{2} \tilde{y}}{d t^{* 2}}+2 n \frac{d \tilde{x}}{d t^{*}}-n^{2} \tilde{y} & =-k^{2}\left[m_{1} \frac{\tilde{y}}{\tilde{r}_{1}^{3}}+m_{2} \frac{\tilde{y}}{\tilde{r}_{2}^{3}}\right]
\end{aligned}
$$

onde, com a introdução da notação $\tilde{r}_{1}$ e $\tilde{r}_{2}$, queremos indicar que no sitema de coordenadas sinódico a distância não depende explicitamente do tempo.

A equação (3.21) verifica nossa intuição de que, em coordenadas sinódicas, é esperado que a função força independa explicitamente do tempo. Ao compararmos a equação (3.9) e a (3.21), percebemos que o lado direito da última ficou mais simples, porém o lado esquerdo ficou um pouco mais complicado devido ao aparecimento de termos de derivada primeira e termos lineares na variável dependente do tempo. Os termos $n^{2} \tilde{x}$ e $n^{2} \tilde{y}$ trazem menores preocupações, pois eles podem ser englobados do lado esquerdo da equação, mas a presença da derivada primeira levanta a questão de quanto a transformação é útil realmente, ou se ela nos trás outros problemas para resolver a equação. A resposta dessa pergunta está relacionada ao fato que a nova equação (3.21) salienta a existência de uma integral útil implicitamente. De fato a única integral conhecida do problema restrito pode ser obtida diretamente de (3.21). Para isto é conveniente mostrar a função potencial da equação (3.21). Portanto escrevemos

$$
\begin{aligned}
& \frac{d^{2} \tilde{x}}{d t^{* 2}}-2 n \frac{d \tilde{y}}{d t^{*}}=\frac{\partial F^{*}}{\partial \tilde{x}}, \\
& \frac{d^{2} \tilde{y}}{d t^{* 2}}+2 n \frac{d \tilde{x}}{d t^{*}}=\frac{\partial F^{*}}{\partial \tilde{y}},
\end{aligned}
$$

e achar a função $F^{*}$. Essa função pode ser obtida diretamente da transformação ou usando teoria de potencial, 


$$
F^{*}=\frac{n^{2}}{2}\left(\tilde{x}^{2}+\tilde{y}^{2}\right)+k^{2}\left(\frac{m_{1}}{\tilde{r}_{1}}+\frac{m_{2}}{\tilde{r}_{2}}\right) .
$$

A equação (3.23) possui uma integral, como pode ser visto, ao multiplicarmos a primeira equação por $d \tilde{x} / d t^{*}$, a segunda por $d \tilde{y} / d t^{*}$, adicionando-as e integrando o resultado com respeito a $t^{*}$. Temos

$$
\frac{1}{2}\left[\left(\frac{d \tilde{x}}{d t^{*}}\right)^{2}+\left(\frac{d \tilde{y}}{d t^{*}}\right)^{2}\right]=\int_{t_{0}^{*}}^{t^{*}}\left[\left(\frac{\partial F^{*}}{\partial \tilde{x}}\right) d \tilde{x}+\left(\frac{\partial F^{*}}{\partial \tilde{y}}\right) d \tilde{y}\right]=F^{*}-C^{*} / 2 .
$$

A integral e $C^{*}$ são conhecidos como a integral Jacobiana e a constante Jacobiana.

\subsubsection{Equações do movimento em coordenadas não físicas}

A equação do movimento no sistema inercial (3.9) contem os parâmetros físicos $k^{2}, a, b, m_{1}$, $m_{2}$, e $n$, os quais não são todos independentes entre si. Agora mostraremos que o problema restrito depende somente de um parâmetro físico, ao usarmos coordenadas adimensionais (não físicas). Para esse propósito usaremos letras gregas (exceto o tempo) para representar as coordenadas:

$$
\begin{array}{r}
\xi=X / l ; \eta=Y / l ; t=n t^{*} ; \mu_{1}=m_{1} / M=a / l \\
\mu_{2}=m_{2} / M=b / l ; \rho_{1}=R_{1} / l ; \rho_{2}=R_{2} / l
\end{array}
$$

As equações do movimento (3.6) ficam

$$
d^{2} \xi / d t^{2}=\partial \phi / \partial \xi, \quad d^{2} \eta / d t^{2}=\partial \phi / \partial \eta
$$

onde

$$
\phi=F / l^{2} n^{2}=\mu_{1} / \rho_{1}+\mu_{2} / \rho_{2}
$$

e

$$
\begin{aligned}
& \rho_{1}^{2}=\left(\xi-\mu_{2} \cos t\right)^{2}+\left(\eta-\mu_{2} \sin t\right)^{2}, \\
& \rho_{2}^{2}=\left(\xi+\mu_{1} \cos t\right)^{2}+\left(\eta+\mu_{1} \sin t\right)^{2} .
\end{aligned}
$$

As variáveis nas equações do movimento são as adimensionais $(\xi, \eta)$ e o tempo $t$. Os únicos parâmetros (constantes) remanescentes são $\mu_{1}$ e $\mu_{2}$, porém, pelo fato de que $m_{1} / M+m_{2} / M=1$, temos que $\mu_{1}+\mu_{2}=1$, isto é, dada uma massa adimensional, a outra fica determinada. Portanto ficamos com apenas um parâmetro (ou $\mu_{1}$ ou $\mu_{2}$ ).

As equações adimensionais correspondentes às equações (3.9) são

$$
\begin{aligned}
& \frac{d^{2} \xi}{d t^{2}}=-\left[\mu_{1} \frac{\left(\xi-\mu_{2} \cos t\right)}{\rho_{1}^{3}}+\mu_{2} \frac{\left(\xi+\mu_{1} \cos t\right)}{\rho_{2}^{3}}\right] \\
& \frac{d^{2} \eta}{d t^{2}}=-\left[\mu_{1} \frac{\left(\eta-\mu_{2} \sin t\right)}{\rho_{1}^{3}}+\mu_{2} \frac{\left(\eta+\mu_{1} \sin t\right)}{\rho_{2}^{3}}\right] .
\end{aligned}
$$

Agora vamos mostrar as equações de movimento no sistema de coordenadas sinódico usando variáveis não físicas. Iremos observar que desse modo obtemos a mais simples das formas para as equações do movimento.

Introduzindo

$$
\begin{array}{r}
x=\tilde{x} / l ; y=\tilde{y} / l ; t=n t^{*} ; \mu_{1}=m_{1} / M=a / l \\
\mu_{2}=m_{2} / M=b / l ; r_{1}=\tilde{r}_{1} / l ; r_{2}=\tilde{r}_{2} / l,
\end{array}
$$

a equação (3.21) fica da seguinte forma 


$$
\begin{aligned}
& \ddot{x}-2 \dot{y}=\tilde{\Omega}_{x}, \\
& \ddot{y}+2 \dot{x}=\tilde{\Omega}_{y},
\end{aligned}
$$

onde os pontos denotam a derivada com relação ao tempo adimensional $t$, e o subescrito à derivada parcial. A função $\bar{\Omega}$ corresponde a

$$
\tilde{\Omega}=\frac{F^{*}}{l^{2} n^{2}}
$$

$\mathrm{ou}$

$$
\tilde{\Omega}=\frac{1}{2}\left(x^{2}+y^{2}\right)+\frac{\mu_{1}}{r_{1}}+\frac{\mu_{2}}{r_{2}},
$$

onde

$$
\begin{aligned}
r_{1}^{2} & =\left(x-\mu_{2}\right)^{2}+y^{2}, \\
r_{2}^{2} & =\left(x+\mu_{1}\right)^{2}+y^{2} .
\end{aligned}
$$

As equações (3.37) e (3.40), as quais definem o problema no sistema de coordenadas sinódicos, são vastamente usadas. Uma modificação de $\tilde{\Omega}$ pela adição de uma constante não afetará as equações de movimento e poderá oferecer maior simetria ao problema. Seja

$$
\Omega=\tilde{\Omega}+\frac{1}{2} \mu_{1} \mu_{2}
$$

o que resulta em

$$
\Omega=\mu_{1}\left(\frac{r_{1}^{2}}{2}+\frac{1}{r_{1}}\right)+\mu_{2}\left(\frac{r_{2}^{2}}{2}+\frac{1}{r_{2}}\right) .
$$

As equações de movimento ficam

$$
\begin{aligned}
& \ddot{x}-2 \dot{y}=\Omega_{x}, \\
& \ddot{y}+2 \dot{x}=\Omega_{y} .
\end{aligned}
$$

A integral Jacobiana da equação (3.37) fica

$$
\dot{x}^{2}+\dot{y}^{2}=2 \Omega-C,
$$

onde $C=\tilde{C}+\mu_{1} \mu_{2}$, em que $\tilde{C}$ é a constante jacobiana associada à $\tilde{\Omega}$.

A integral Jacobiana (3.46) no sistema de coordenadas sinódicos adimensional conecta a velocidade relativa adimensional com a posição através da constante Jacobiana.

Para retornar para o sistema inercial (sideral adimensional) devemos aplicar a transformação

$$
\begin{aligned}
& \xi=x \cos t-y \sin t, \\
& \eta=x \sin t+y \cos t .
\end{aligned}
$$

Os parâmetros $\mu_{1}$ e $\mu_{2}$ relacionam-se da seguinte forma: $\mu_{1}+\mu_{2}=1$. No sistema de variáveis adimensionais, $\mu_{1}$ e $\mu_{2}$ representam as massas normalizadas das primárias, cuja a soma, portanto, é o valor unitário e cuja a distância também é unitária pois as dimensões lineares que ocorrem nas equações são transformadas em adimensionais dividindo pela distãncia entre as primárias $l$. $\mathrm{O}$ tempo adimensional, $t$, também é uma medida do ângulo pelo qual o sistema rotacional gira durante o tempo $t^{*}$. Outra interpretação para $t$ é a longitude da primária de massa $m_{1}$.

As variáveis dependentes $x$ e $y$ referem-se ao sistema rotacional; caso elas sejam constantes a interpretação física disso é que o terceiro corpo (infinitesimal) tem posição fixa no sistema de coordenadas rotacional, isto é, ele se move em círculos com velocidade constante no sistema fixo. O movimento médio do sistema rotacional adimensional tem valor unitário também (a velocidade 
angular é igual a 1) e a constante gravitacional é $k^{2}=1$. A figura (3.2) mostra o sistema de coordenadas adimensional.

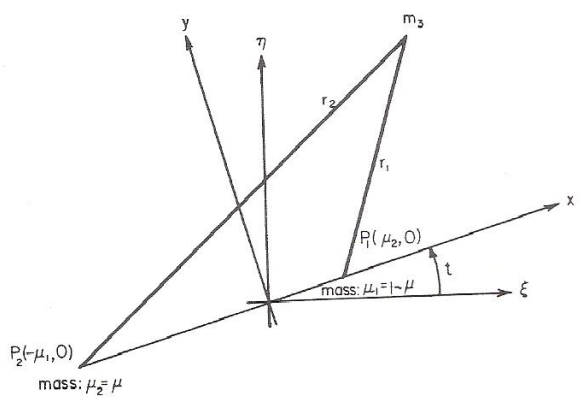

Figura 3.2: Configuração em coordenadas inercial $(\xi, \eta)$ e rotacional $(x, y)$ nas variáveis não físicas $\left(\mu_{1}>\right.$ $\left.\mu_{2}\right)$.

Como já foi mencionado anteriormente, as equações (3.44) e (3.45) contém apenas um parâmetro. Deste modo para eliminarmos ou $\mu_{1}$ ou $\mu_{2}$, usaremos a relação $\mu_{1}=1-\mu_{2}$. Deste modo chamaremos $\mu_{2}=\mu$, então $\mu_{1}=1-\mu$. O valor da massa adimensional $\mu_{2}$ está no intervalo $I=[0,1]$. Os valores $\mu=0$ ou $\mu=1$ correspondem a massa (apenas uma primária) unitária na origem. O valor $\mu=1 / 2$ corresponde a dois corpos de massas iguais localizados em $x= \pm \frac{1}{2}$, os dois tendo massas adimensionais de valor $1 / 2$. Quando $0 \leq \mu<1 / 2$ a massa maior (dentre as primárias) está localizada em $0 \leq x<1 / 2$ e a massa menor (dentre as primárias) está em $-1 \leq x<-1 / 2$, e quando $1 / 2<\mu \leq 1$ a massa maior está em $-1 / 2<x \leq 0$ e a menor em $1 / 2<x \leq 1$. O caso em que $\mu=1 / 2$ corresponde a simetria entre as primárias. A literatura está dividida em relação a onde posicionar a massa maior, e a escolha da convenção a ser seguida, porém, devido às características gerais das equações (3.44) e (3.45), essa escolha não importa muito, pois podemos substituir $\mu$ por $1-\mu$ e as equações acabam sendo iguais, não importa onde posicionamos a massa maior ou menor das primárias.

\subsubsection{Relação com o problema geral dos três corpos}

A aproximação para um movimento circular dos planetas em torno do Sol, e as pequenas massas dos satélites dos planetas e dos asteróides comparada às massas dos planetas, originalmente sugere a formulação do problema restrito.

Foi visto que a energia total dos três corpos no problema restrito não é conservada. Por outro lado é conhecido que no problema dos três corpos gerais a energia total é conservada. Portanto, na transição do problema geral para o restrito, o princípio da conservação de energia é perdido. O problema geral dos três corpos tem apenas poucas aplicaçoes diretas em mecânica celeste e dinâmica orbital, e o conhecimento sobre comportamento das soluções do problema geral é muito menor que o do problema restrito. A abordagem que daremos aqui, sobre o problema dos três corpos geral, tem o propósito de mostrar sua relação com o problema restrito, e não de dar um tratamento qualitativo ao problema.

O primeiro reconhecimento desses fatos foi obtido por Euler (1772). Após ele, várias publicações trataram o assunto.

O problema dos três corpos é definido como: tem-se três partículas com massas arbitrárias sobre influência de suas respectivas forças de atração de acordo com a lei da gravitação de Newton; elas são livres para se mover no espaço e inicialmente podem se mover de qualquer maneira. A diferença entre esse problema e o restrito é, primeiramente, que no caso restrito apenas duas massas são arbitrárias; a terceira é muito menor que as outras duas. O conjunto das condições iniciais do problema geral é livre; no restrito as massas significativas estão em movimento circular.

A figura (3.3) mostra a notação convencional. As massas dos três corpos são $m_{1}, m_{2}, m_{3}$, e seus 
vetores posições são

$$
r_{1}=\left(q_{1}, q_{2}, q_{3}\right), r_{2}=\left(q_{4}, q_{5}, q_{6}\right), r_{3}=\left(q_{7}, q_{8}, q_{9}\right) .
$$

Os vetores que ligam as massas são

$$
r_{12}=r_{1}-r_{2}, r_{23}=r_{2}-r_{3}, r_{31}=r_{3}-r_{1},
$$

e a distância entre as massas são seus módulos $\left|r_{12}\right|,\left|r_{23}\right|,\left|r_{31}\right|$.

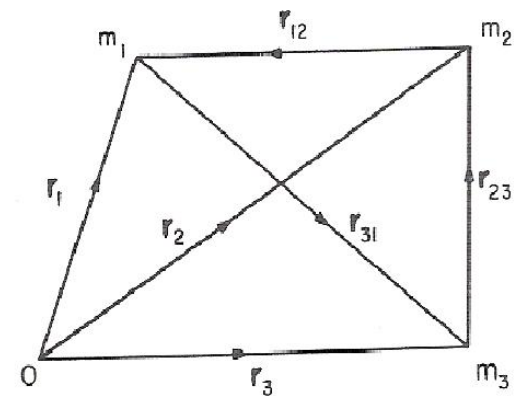

Figura 3.3: $O$ problema geral dos três corpos; as massas são $m_{i}$ e os vetores posições $r_{i}$.

A função potencial é

$$
F=k^{2}\left(\frac{m_{1} m_{2}}{\left|r_{12}\right|}+\frac{m_{3} m_{2}}{\left|r_{23}\right|}+\frac{m_{1} m_{3}}{\left|r_{31}\right|}\right)
$$

e as equações do movimento são

$$
m_{i} \ddot{r}_{i}=\partial F / \partial r_{i}, i=1,2,3,
$$

$\mathrm{Ou}$

$$
\begin{aligned}
\ddot{r}_{1} & =-k^{2} m_{2} \frac{r_{1}-r_{2}}{\left|r_{1}-r_{2}\right|^{3}}+k^{2} m_{3} \frac{r_{3}-r_{1}}{\left|r_{3}-r_{1}\right|^{3}}, \\
\ddot{r}_{2} & =-k^{2} m_{3} \frac{r_{2}-r_{3}}{\left|r_{2}-r_{3}\right|^{3}}+k^{2} m_{1} \frac{r_{1}-r_{2}}{\left|r_{1}-r_{2}\right|^{3}}, \\
\ddot{r}_{3} & =-k^{2} m_{1} \frac{r_{3}-r_{1}}{\left|r_{3}-r_{1}\right|^{3}}+k^{2} m_{2} \frac{r_{2}-r_{3}}{\left|r_{2}-r_{3}\right|^{3}} .
\end{aligned}
$$

O sistema consiste de três equações diferenciais de segunda ordem nas coordenadas $r_{i}$, ou seja, nove equações diferenciais de segunda ordem nas variáveis $q_{i}$. Isso resulta num sistema de primeira ordem de 18 equações enquanto que no problema restrito tem-se quatro. Isso mostra o quão mais complexo é o problema geral.

O sistema de ordem dezoito pode ser reduzido até ordem seis, e por ser uma redução nada trivial, a redução do problema geral dos três corpos é um dos assuntos principais na literatura clássica sobre esse assunto.

Como nenhuma força externa age no sistema, isto é, $\sum_{i=1}^{3} m_{i} \ddot{r}_{i}=0$, o centro de massa move-se em uma linha reta com velocidade constante, ou seja,

$$
\sum_{i=1}^{3} m_{i} \dot{r}_{i}=a e \sum_{i=1}^{3} m_{i} r_{i}=a t+b .
$$

Essas duas equações vetoriais, correspondentes a seis equações escalares com seis constantes de integração escalares, representam seis integrais com as quais o sistema pode ser reduzido de ordem dezoito para ordem doze. 
A conservação do momento angular pode ser escrita como

$$
\sum_{i=1}^{3} r_{i} \times m_{i} \dot{r}_{i}=c .
$$

Quando as três integrais escalares são usadas em conjunto com as coordenadas ignoráveis introduzidas, o sistema é reduzido de ordem doze para ordem oito.

A última redução (de oito para seis) é possível se usarmos a integral da energia e eliminarmos o tempo. A integral da energia é obtida das equações (3.49) pelo método usual.

Neste momento podemos mostrar como o problema geral é reduzido ao problema restrito.

As equações (3.50) descrevem o caso geral do problema dos três corpos com forças gravitacionais Newtonianas. A estrutura dessas equações é de algum interesse, na medida que as massas $m_{1}, m_{2}$ e $m_{3}$ são negligenciadas das equações um, dois e três respectivamente. Esse fato não "desacopla"as equações, já que todos os três vetores posição ocorrem nas três equações.

Os termos do lado direito têm significado físico específico. O primeiro termo do lado direito da primeira equação, por exemplo, representa a força, por unidade de massa, agindo na primeira massa devido a presença da massa 2 . O segundo termo do mesmo lado da mesma equação é o efeito da terceira massa $m_{3}$ na primeira $m_{1}$.

Diminuindo a terceira massa, reduziremos sua influência sobre as outras duas, isto é, quando $m_{3} \longrightarrow 0$ as duas primeiras equações de (3.50) aproximam-se de $\left(k^{2}=1\right)$

$$
\begin{aligned}
& \ddot{r}_{1}=-m_{2} \frac{r_{1}-r_{2}}{\left|r_{1}-r_{2}\right|^{3}}, \\
& \ddot{r}_{2}=-m_{1} \frac{r_{2}-r_{1}}{\left|r_{2}-r_{1}\right|^{3}},
\end{aligned}
$$

enquanto a terceira equação não se altera. Esse fato agora desacopla as equações, já que, o movimento das primárias podem ser determinados agora, sem considerar o efeito da terceira massa. De fato, as equações (3.51) são as equações do problema dos dois corpos.

A terceira equação de (3.50) requer um comentário adicional. Como nas duas primeiras equações adotou-se $m_{3}=0$ (gerando 3.51), então a terceira equação se reduz $0=0$, pois neste caso, $m_{3}=0$, e em (3.49) nenhuma divisão por $m_{3}$ é permitida. No próximo parágrafo usaremos esse fato para fazer uma aproximação do problema geral dos três corpos, pois $m_{3} \neq 0$, mas essa massas será pequena o suficiente para que suponhamos que seja nula. Essa aproximação será o problema restrito

A hipótese feita é que $m_{3} \neq 0$, mas $m_{3}$ é suficientemente pequena de modo que o movimento das primárias não são afetados. Isto é, a equação (3.51) é uma aproximação, enquanto a terceira equação de (3.50) é exata. O sistema de equações consistindo de (3.51) mais a terceira equação de (3.50) representa o nosso sistema dinâmico somente aproximadamente; o grau de aproximação é dado pelo o quão pequeno os seguintes termos:

$$
a=m_{3} \frac{r_{3}-r_{1}}{\left|r_{3}-r_{1}\right|^{3}} \text { e } b=m_{3} \frac{r_{2}-r_{3}}{\left|r_{2}-r_{3}\right|^{3}}
$$

serão em comparação com

$$
a^{\prime}=m_{2} \frac{r_{1}-r_{2}}{\left|r_{1}-r_{2}\right|^{3}} \text { e } b^{\prime}=m_{1} \frac{r_{1}-r_{2}}{\left|r_{1}-r_{2}\right|^{3}},
$$

respectivamente. Ou seja, queremos que as razões $a / a^{\prime}$ e $b / b^{\prime}$ sejam pequenas o suficiente.

O efeito das primárias no movimento da infinitesimal é dado pela terceira equação de (3.50). Aceitando essa aproximação antes mencionada, pode-se resolver a equação (3.51), e substituir a solução na terceira equação de (3.50), e obter a equação diferencial de sexta ordem do movimento do terceiro corpo: 


$$
\ddot{r}_{3}=-k^{2} m_{1} \frac{r_{3}-r_{1}}{\left|r_{3}-r_{1}\right|^{3}}+k^{2} m_{2} \frac{r_{2}-r_{3}}{\left|r_{2}-r_{3}\right|^{3}},
$$

onde agora $r_{1}$ e $r_{2}$ são funções dadas, dependentes do tempo e das condições iniciais das primárias.

A equação (3.52) descreve o problema dos três corpos restrito ou, mais explicitamente, o movimento da infinitesimal no problema dos três corpos restrito. A "restrição"é equivalente à hipótese que o movimento das primárias não é influenciado pela infinitesimal, enquanto o da mesma é determinado pelas massas e movimentos das primárias.

\subsubsection{Classificação e modificações no problema restrito}

O último resultado da seção anterior, nos permite apresentar várias generalizações do problema restrito. A equação (3.52) pode ser escrita como

$$
\ddot{r}_{3}=f\left(m_{1}, m_{2}, r_{1}, r_{2}, r_{3}\right),
$$

onde $r_{1}$ e $r_{2}$ são soluções do problema dos dois corpos, determinadas pelas condições iniciais dada para as primárias. A função $f$ representa o campo de forças agindo em $m_{3}$ o qual na equação (3.52) é o campo gravitacional Newtoniano devido às primárias. Deste ponto de vista a forma mais geral do problema restrito é

$$
\ddot{r}=g(r, t),
$$

isto é, o problema é a determinação do movimento de um corpo com vetor posição $r$ sob a influência de um campo $g$ dependente da posição do corpo e do tempo. A equação (3.54) descreve um sistema de dois ou três graus de liberdade dependendo da dimensão do problema ser dois (plano) ou três (espaço).

O problema introduzido até aqui como problema restrito especifica que duas massas $\left(m_{1}\right.$ e $\left.m_{2}\right)$ estão em movimento circular e também requer que a infinitesimal esteja no plano definido pelos outros dois corpos.

Podemos fazer outras "restrições"aos movimentos dos corpos, com vista a estudar outros problemas:

1. O movimento das primárias $\left(m_{1}\right.$ e de $\left.m_{2}\right)$ não é circular. Se forças gravitacionais Newtonianas são envolvidas no problema dos dois corpos, dependendo das condições iniciais, a solução pode ser qualquer cônica. o caso mais importante é quando os dois corpos se movem em elipses. Esse caso, problema restrito elíptico, ou pseudo problema restrito, tem muitas aplicações.

2. O problema restrito tridimensional se preocupa com o movimento da infinitesimal, que, neste caso, não está contido no plano das primárias. Esse problema aparece quando as condições iniciais do terceiro corpo são tais que o terceiro corpo está fora do plano das primárias, ou quando sua velocidade inicial tem uma componente fora deste mesmo plano.

3. O valor da razão $m_{1} / m_{2}$ e do parâmetro $\mu$ têm efeito importante sobre o movimento do terceiro corpo assim como na aproximação do problema. O valor $\mu=0$ muda o problema restrito para o problema dos dois corpos, portanto o problema com baixos valores de $\mu$ aparece como problema de perturbações do problema dos dois corpos. Por outro lado com $\mu=1 / 2$, temos um caso particular do problema dos três corpos, neste caso recebe um nome especial, problema de Copenhagen.

4. Se as massas das primárias dependem do tempo, as equações do movimento mudam significantemente, mas as idéias básicas do problema restrito continuam úteis em dinâmica estelar e cosmogonia.

5. Se as forças envolvidas não forem centrais, uma pergunta importante que se pode fazer é em qe situação a hipótese de os movimentos das primárias serem circulares ou cônicos continuam 
úteis. Em outras palavras, como no caso de forças centrais queremos que as primárias descrevam soluções de configurações centrais (movimento circular ou cônico), para então estudar o movimento da infinitesimal no caso em que a força não é central, que tipo de solução das primárias permite estudar de modo análogo o movimento da infinitesimal?

\subsection{Descrição do problema espacial}

O problema tratado até este momento foi o problema restrito planar, em que duas massas, chamadas primárias, giram em torno do seu centro de massa em órbitas circulares e no plano do movimento das primárias ocorre o movimento da terceira massa (infinitesimal), que não influencia o movimento das primárias. Supondo forças gravitacionais Newtonianas, o intuito era estudar o comportamento da terceira massa. A partir de agora vamos comentar uma modificação que se pode fazer a esse problema, que chamaremos de Problema restrito tridimensional.

\subsubsection{Formulação do problema e equação do movimento}

Neste caso as primárias continuam contidas num plano girando em torno do seu centro de massas em círculos. Os corpos estão no eixo de rotação $x$, localizados em $P_{1}(\mu, 0,0)$ e $P_{2}(\mu-1,0,0)$. O eixo $y$ está localizado perpendicular ao eixo $x$ e está no plano de rotação das primárias. O plano $x y$ gira com movimento médio igual a 1 ; o vetor velocidade angular é paralelo ao eixo $z$, o qual forma o sistema da "mão direita"com os eixos $x$ e $y$, ver figura (3.4)

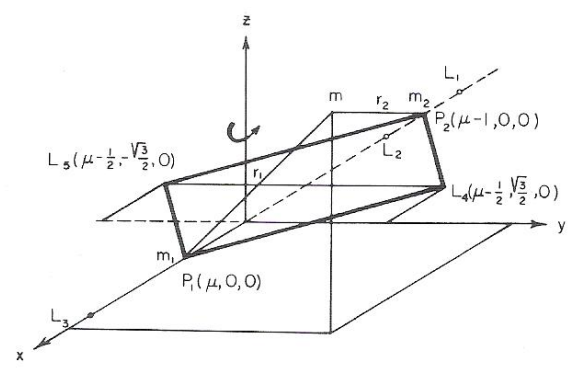

Figura 3.4: O problema restrito tridimensional, e seu pontos de equilíbrio $L_{i}$.

As equações do movimento da terceira partícula são

$$
\begin{gathered}
\ddot{x}-2 \dot{y}=\Omega_{x}, \\
\ddot{y}+2 \dot{x}=\Omega_{y}, \\
\ddot{z}=\Omega_{z},
\end{gathered}
$$

onde

$$
\Omega=\frac{1}{2}\left(x^{2}+y^{2}\right)+\frac{1-\mu}{r_{1}}+\frac{\mu}{r_{2}}+\frac{1}{2} \mu(1-\mu),
$$

com

$$
\begin{gathered}
r_{1}^{2}=(x-\mu)^{2}+y^{2}+z^{2}, \\
r_{2}^{2}=(x-\mu+1)^{2}+y^{2}+z^{2} .
\end{gathered}
$$

Note que essas definições para $r_{1}$ e $r_{2}$ contêm a coordenada $z$, portanto agora

$$
\frac{1}{2}\left(x^{2}+y^{2}\right)+\frac{1}{2} \mu(1-\mu) \neq \frac{1}{2}\left[(1-\mu) r_{1}^{2}+\mu r_{2}^{2}\right] .
$$

Consequentemente a forma elegante e simétrica da função $\Omega$ usada no caso planar não é aplicável nas equações (3.55). Neste caso definiremos a função 


$$
\tilde{\Omega}=\frac{1}{2}\left[(1-\mu) r_{1}^{2}+\mu r_{2}^{2}\right]+\frac{1-\mu}{r_{1}}+\frac{\mu}{r_{2}},
$$

que no caso tridimensional torna-se

$$
\tilde{\Omega}=\frac{1}{2}\left(x^{2}+y^{2}+z^{2}\right)+\frac{1}{2} \mu(1-\mu)+\frac{1-\mu}{r_{1}}+\frac{\mu}{r_{2}},
$$

Como $\tilde{\Omega}_{z}$ neste caso é dado por

$$
\tilde{\Omega}_{z}=z+\Omega_{z},
$$

as novas equações de movimento ficam da seguinte forma

$$
\begin{gathered}
\ddot{x}-2 \dot{y}=\tilde{\Omega}_{x}, \\
\ddot{y}+2 \dot{x}=\tilde{\Omega}_{y}, \\
\ddot{z}+z=\tilde{\Omega}_{z} .
\end{gathered}
$$

A integral Jacobiana que pode ser obtida é da seguinte forma

$$
\dot{x}^{2}+\dot{y}^{2}+\dot{z}^{2}=2 \Omega(x, y, z)-C .
$$

A formulção Hamiltoniana para o caso tridimensional é igualmente simples. Considere um sistema de coordenadas fixado com variáveis adimensionais, como em $(3.27),(\xi, \eta, \zeta)(\zeta=Z / l$, aqui). Como coordenadas generalizadas tomamos $\left(Q_{1}, Q_{2}, Q_{3}\right)=(\xi, \eta, \zeta)$. Portanto o Hamiltoniano correspondente é

$$
\mathcal{H}=\frac{1}{2}\left(P_{1}^{2}+P_{2}^{2}+P_{3}^{2}\right)-\phi\left(Q_{1}, Q_{2}, Q_{3}, t\right),
$$

onde

$$
P_{1}=\dot{Q}_{1}=\dot{\xi}, P_{2}=\dot{Q}_{2}=\dot{\eta}, P_{3}=\dot{Q}_{3}=\dot{\zeta}
$$

e

$$
\phi=\mu_{1} / \rho_{1}+\mu_{2} / \rho_{2} .
$$

Note que a definição de $\rho_{i}$ é levemente diferente do caso bidimensional, já que agora

$$
\begin{aligned}
& \rho_{1}^{2}=\left(Q_{1}-\mu_{2} \cos (t)\right)^{2}+\left(Q_{2}-\mu_{2} \sin (t)\right)^{2}+Q_{3}^{2}, \\
& \rho_{2}^{2}=\left(Q_{1}+\mu_{1} \cos (t)\right)^{2}+\left(Q_{2}+\mu_{1} \sin (t)\right)^{2}+Q_{3}^{2},
\end{aligned}
$$

onde $\mu_{1}=1-\mu$ e $\mu_{2}=\mu$.

No sistema de coordenadas sinódicas o Hamiltoniano é

$$
\tilde{\mathcal{H}}=\frac{1}{2}\left(p_{1}^{2}+p_{2}^{2}+p_{3}^{2}\right)+q_{2} p_{1}-q_{1} p_{2}-\tilde{F}\left(q_{1}, q_{2}, q_{3}\right) .
$$

As relações entre as coordenadas e momentos sinódicos e inercial são

$$
\begin{aligned}
& q=A^{T} Q, \\
& P=A^{T} p,
\end{aligned}
$$

onde $A^{T}$ é a transposta da matriz

$$
A=\left(\begin{array}{ccc}
\cos (t) & -\sin (t) & 0 \\
\sin (t) & \cos (t) & 0 \\
0 & 0 & 1
\end{array}\right)
$$

A função potencial é 


$$
\tilde{F}=\mu_{1} / \rho_{1}+\mu_{2} / \rho_{2}
$$

onde

$$
\begin{aligned}
& \rho_{1}^{2}=\left(q_{1}-\mu_{2}\right)^{2}+q_{2}^{2}+q_{3}^{2}, \\
& \rho_{2}^{2}=\left(q_{1}+\mu_{1}\right)^{2}+q_{2}^{2}+q_{3}^{2} .
\end{aligned}
$$

\subsection{Configurações centrais}

O problema do $N$ corpos tem resistido a todas as tentativas de ser resolvido; de fato acredita-se que o problema não é integrável no sentido clássico. Com o passar dos anos, várias soluções especiais foram encontradas usando técnicas matemáticas especiais para situações particulares do problema. Nesta seção vamos abordar rapidamente uma dessas técnicas, chamada de técnica da configuração central.

Soluções do tipo configuração central são importantes em mecânica celeste por várias razões. Quando os corpos do problema dos $N$ corpos tendem a colisões ou a expandir ao infinito, eles tendem a ficar com um configuração central. Para nós, a configuração central passa a ser a mais importante, pois, além de aparecerem órbitas periódicas naturalmente, ela é uma configuração exigida para formular o problema restrito dos $(N+1)$ corpos, em que as $k \leq N$ primárias devem estar em configuração central para podermos estabelecer o problema.

\subsubsection{Soluções de equilíbrio}

A mais simples das soluções é o equilíbrio. Um equilíbrio deve satisfazer

$$
\frac{\partial U}{\partial q_{i}}=0, i=1, \ldots, N,
$$

em que $U$ é o potencial, que no caso é homogêneo de grau -1 .

Entretanto, por $U$ ser homgênea de grau -1 e pelo teorema de Euler sobre funções homogêneas,

$$
\sum q_{i} \frac{\partial U}{\partial q_{i}}=-U
$$

Como $U$ é uma soma de termos estritamente positivos, não há pontos de equilíbrio no problema dos $N$ corpos.

\subsubsection{Equações para a configuração central}

Para procurarmos soluções colineares de (2.5), tentamos $q_{i}(t)=\phi(t) a_{i}$, onde $a_{i}$ são vetores constante do $\mathbb{R}^{2}$ ou $\mathbb{R}^{3}$ e $\phi(t)$ é uma função escalar. Substituindo em (2.5) e rearranjando temos

$$
|\phi|^{3} \phi^{-1} \ddot{\phi} m_{i} a_{i}=\sum_{j=1, j \neq i}^{N} \frac{m_{i} m_{j}\left(a_{j}-a_{i}\right)}{\left\|a_{j}-a_{i}\right\|^{3}} .
$$

Como o lado direito é constante, o lado esquerdo tem que ser constante, portanto (3.61) tem uma solução se existir uma função escalar $\phi(t)$, uma constante $\lambda$, e vetores constantes $a_{i}$ tal que

e

$$
\ddot{\phi}=-\frac{\lambda \phi}{|\phi|^{3}}
$$

$$
-\lambda m_{i} a_{i}=\sum_{j=1, j \neq 1}^{N} \frac{m_{i} m_{j}\left(a_{j}-a_{i}\right)}{\left\|a_{j}-a_{i}\right\|^{3}}, i=1, \ldots, N .
$$


A equação (3.62) é uma simples equação diferencial e portanto tem várias soluções (dependendo da condição inicial). Por exemplo, uma solução é $\alpha t^{2 / 3}$, onde $\alpha^{3}=9 \lambda / 2$. A equação (3.63) é um sistema não trivial equações algébricas. As soluções completas são conhecidas apenas para o caso $N=2,3$, mas há várias soluções especiais conhecidas. Uma configuração das $N$ partículas dada pelos vetores constantes $a_{i}, i=1, \ldots, N$ satisfazendo (3.63) para algum $\lambda$ é chamada Configuração Central que, para encurtar, chamaremos de c.c.

Note que qualquer graduação uniforme de um c.c. é também um c.c. Deste modo para medirmos o tamanho do sistema de $N$ corpos, definimos o momento de inércia do sistema como

$$
I=\frac{1}{2} \sum_{i=1}^{N} m_{i}\left\|q_{i}\right\|^{2} .
$$

Agora podemos reescrever (3.63) como

$$
\frac{\partial U}{\partial q}(a)+\lambda \frac{\partial I}{\partial q}(a)=0
$$

onde $q=\left(q_{1}, \ldots, q_{N}\right)$ e $a=\left(a_{1}, \ldots, a_{N}\right)$. A constante $\lambda$ pode ser considerada como um multiplicador de Lagrange, potanto uma configuração central é um ponto crítico do potencial $U$ restrito a um vínculo, que é a variedade do momento de inércia constante dado, $I=I_{0}$. Fixando $I_{0}$ fixa-se a graduação.

Seja $a$ uma configuração central. $U$ é homogênea de grau -1 e $I$ homogênea de grau 2 . Tomando o produto escalar de (3.65) com $a$ e aplicando o teorema de Euler sobre funções homogêneas temos

$$
-U+2 \lambda I=0
$$

$\mathrm{Ou}$

$$
\lambda=\frac{U(a)}{2 I(a)}>0
$$

\subsubsection{Equilíbrio relativo}

Considere agora o caso do problema dos $N$ corpos planar, portanto todos os vetores estão em $\mathbb{R}^{2}$. Identificamos $\mathbb{R}^{2}$ com o plano complexo $\mathbb{C}$, considerando $q_{i}, p_{i}$ como números complexos. Procura-se uma solução homográfica (alegórica) de (2.5) fazendo $q_{i}(t)=\phi(t) a_{i}$, onde $a_{i}$ são constantes complexas, e $\phi(t)$ é uma função a valores complexos. Geometricamente, multiplicação por um número complexo, significa rotacionar e dilatar ou comprimir, isto é, fazer uma homografia.

Procuramos soluções tais que a configuração das partículas seja sempre homograficamente equivalente a uma configuração central. Pensando dessa forma em (2.5) e rearranjando os termos, temos (3.61), e o mesmo argumento nos dá (3.62), que agora é o problema de Kepler bidimensional, e a equação (3.63). Isto é, se tivermos uma solução de (3.63) com os $a_{i}$ planares, então existe uma solução do problema dos $N$ corpos da forma $q_{i}=\phi(t) a_{i}$, onde $\phi(t)$ é qualquer solução do problema de Kepler planar.

Considere o problema dos $N$ corpos nas coordenadas rotacionais $Q_{1}, \ldots, Q_{N}, P_{1}, \ldots, P_{N}$. Uma solução de equilíbrio $Q_{i}=a_{i}, P_{i}=b_{i}$, do problema dos $N$ corpos em coordenadas rotacionais é chamada equilíbrio relativo e deve satisfazer

$$
\begin{gathered}
\frac{b_{i}}{m_{i}}+\omega J a_{i}=0, \\
\sum_{j=1, j \neq i}^{N} \frac{m_{i} m_{j}\left(a_{i}-a_{j}\right)}{\left\|a_{i}-a_{j}\right\|^{3}}+\omega J b_{i}=0, i=1, \ldots, N,
\end{gathered}
$$


e portanto os $a_{i}$ devem satisfazer

$$
-\omega^{2} m_{i} a_{i}=\sum_{j=1, j \neq i}^{N} \frac{m_{i} m_{j}\left(a_{i}-a_{j}\right)}{\left\|a_{i}-a_{j}\right\|^{3}}, i=1, \ldots, N .
$$

Como se pode perceber, esta equação é igual a (3.63) $\operatorname{com} \lambda=\omega^{2}$. Portanto uma configuração central planar dá origem a um equilíbrio relativo.

As raízes características da linearização dessas equações do movimento em torno de um equilíbrio relativo são chamadas expoentes do equilíbrio relativo e o polinômio característico de polinômio característico do equilíbrio relativo.

\subsection{Descrição do problema restrito dos $(N+1) \operatorname{corpos}$}

Considere o problema de $(N+1)$ corpos com Hamiltoniano $\tilde{\mathcal{H}}_{N+1}$, em coordenadas rotacionais $(Q, P)$, onde as partículas estão indexadas de 0 até $N$, e faça uma das massas ser pequena, digamos $m_{0}=\epsilon^{2}$. Então o Hamiltoniano é

$$
\tilde{\mathcal{H}}_{N+1}=\left\|P_{0}\right\|^{2} / 2 \epsilon^{2}-Q_{0}^{T} J P_{0}-\sum_{j=1}^{N} \frac{\epsilon^{2} m_{j}}{\left\|Q_{j}-Q_{0}\right\|}+\tilde{\mathcal{H}}_{N}
$$

onde $\tilde{\mathcal{H}}_{N}$ é o Hamiltoniano do problema dos $N$ corpos (primárias) com partículas indexadas de 1 a $N$.

Escolha as primárias de tal forma que elas estejam em alguma configuração central planar do problema dos $N$ corpos, digamos $\left(a_{1}, \ldots, a_{N}\right)$. Seja $Z=\left(Q_{1}, \ldots, Q_{N} ; P_{1}, \ldots, P_{N}\right)$ e $Z^{*}=$ $\left(a_{1}, \ldots, a_{N} ;-m_{1} J a_{1}, \ldots,-m_{N} J a_{N}\right)$, portanto $Z^{*}$ é um equilíbrio relativo. Aqui usaremos a configuração central de tal modo que ela tenha frequência $\omega$ igual a 1. Pelo teorema de Taylor temos

$$
\tilde{\mathcal{H}}_{N}(Z)=\tilde{\mathcal{H}}_{N}\left(Z^{*}\right)+\frac{1}{2}\left(Z+Z^{*}\right)^{T} S\left(Z+Z^{*}\right)+O\left(\left\|Z-Z^{*}\right\|^{3}\right),
$$

onde $S$ é a Hessiana de $\tilde{\mathcal{H}}_{N}$ no ponto $Z^{*}$.

Considere a mudança de variável

$$
Q_{0}=\alpha, P_{0}=\epsilon^{2} \beta, Z=Z^{*}-\epsilon V .
$$

Agora $Q_{i}=a_{i}+O(\epsilon)$. Essa mudança de variáveis é simplética (transformação canônica) com multiplicador $\epsilon^{2}$ e o Hamiltoniano nas novas variáveis será

$$
\tilde{\mathcal{H}}_{N+1}=\left[\|\beta\|^{2} / 2-\alpha^{T} J \beta-\sum_{j=1}^{N} \frac{m_{j}}{\left\|a_{j}-\alpha\right\|}\right]+\left[\frac{1}{2} V^{T} S V+O(\epsilon)\right] .
$$

Dessa forma, ao diminuirmos $|\epsilon|$, ou seja, deixarmos ele ser suficientemente pequeno, o Hamiltoniano do problema dos $(N+1)$ corpos se separa em dois Hamiltonianos, o Hamiltoniano do problema restrito dos $(N+1)$ corpos e o Hamiltoniano da linearização do problema $\operatorname{dos} N$ corpos em torno do equilíbrio $Z^{*}$,

$$
H_{L}=\frac{1}{2} V^{T} S V
$$

Passando ao limite, temos o caso $\epsilon=0$, e as equações do movimentos são

$$
\dot{\alpha}=J \alpha+\beta,
$$




$$
\dot{\beta}=J \beta-\sum_{j=1}^{N} \frac{m_{j}\left(a_{j}-\alpha\right)}{\left\|a_{j}-\alpha\right\|^{3}},
$$

e

$$
\dot{V}=J S V .
$$

Para o problema de uma única massa pequena, dentre as $N+1$, essas são as equações da primeira aproximação. 


\section{Capítulo 4}

\section{Alternância de estabilidade e instabilidade na família do movimento vertical}

Neste capítulo, aplicaremos resultados de capítulos anteriores para estudarmos a estabilidade no problema restrito tridimensional, que passaremos a chamar de Problema de Sitnikov circular (ou PSC) por motivos históricos, embora alguns autores prefiram chamá-lo de "problema de Pavanini"ou "problema de MacMillan".

Aqui estaremos interessados na estabilidade do movimento vertical da massa $m_{3}$ (infinitesimal). Dependendo das condiçõs iniciais, existem três possibilidades de movimento vertical no PSC: o Hiperbólico (a infinitesimal escapa para o infinito com velocidade não nula), Parabólico (escapa para o infinito com velocidade nula) e Periódico (se distancia do baricentro das primárias até um valor finito fixado, que chamaremos de $a$, e depois retorna ao plano, e repete isso com uma certa frequência).

Quando falamos da estabilidade no movimento vertical, estamos interessados em estudar a interferência de perturbações nas condições iniciais que possam alterar o movimento da infinitesimal, ou melhor, em estudar se podemos "mover" $m_{3}$ em um plano paralelo ao plano das primárias de modo que o movimento periódico original, ao ser perturbado dessa forma, continua periódico ou escapa para o infinito. Ou seja, a pergunta que se faz é: Considerando perturbações horizontais $\epsilon$ na posição inicial da infinitesimal, para qual altura inicial $a=q_{3}(0)$ o movimento é estável (ou instável)?

Muitos autores tentaram, alguns com sucesso outros com alguns erros de cálculo, responder a essa pergunta. Os primeiros a tentarem foram Perdios e Markellos, [PM88], e obtiveram a conclusão errada de que o movimento vertical era sempre instável (eles analisaram apenas movimentos verticais com condições iniciais $a<4$ ). Belbruno, [BLO94], apontou esse erro, em seu artigo, foi mostrado pela primeira vez, numericamente, a existência da alternância entre estabilidade e instabilidade no movimento vertical no caso em que a amplitude $a$ é continuamente variada. Depois, Perdios, [Per07], e Soulis, [SBD07], confirmaram a existência, computacionalmente, dessa alternância. Em vista disso, Soulis, [SBD07], propõe a hipótese de que os comprimentos dos intervalos de estabilidade e instabilidade são limitados quando $a$ cresce, essa hipótese foi formulada para $a \in(5,13)$. Na verdade, aqui discutiremos o fato de que, quando $a$ cresce, os comprimentos dos intervalos tendem a ficar cada vez menores, tendendo a zero.

Há mais uma importante propriedade que ocorre para $a>>1$ : começam a aparecer intervalos de instabilidade chamados de "selas complexas"(ver seção 4.3), isto é, todos os autovalores da matriz de monodromia são complexos e não estão no círculo unitário. De acordo com resultados obtidos por Sidorenko, [Sid11], tais intervalos começam a ocorrer a partir de $a \approx 546,026$, e seu comprimento é de $\approx 10^{-5}$. Isso prova que Belbruno, [BLO94], estavam errados também ao dizer que os índices de estabilidade do movimento vertical são sempre reais.

Analisaremos essa pergunta para $a>>1$, no caso, $a>546$, com base no trabalho de Sidorenko, 
[Sid11]. Para $0<a \leq 13$, Soulis, [SBD07], mostraram que há uma alternância entre intervalos de estabilidade e instabilidade, ou seja, existe uma divisão do intervalo $(0,13)$ em subintervalos $\left(a_{0}, b_{0}\right)$, $\left(b_{0}, a_{1}\right), \ldots$, e a alternância de estabilidade e instabilidade diz que se $a \in\left(a_{0}, b_{0}\right)$ o movimento periódico é estável, no próximo intervalo $\left(b_{0}, a_{1}\right)$ o movimento é instável, e assim por diante.

Tanto o resultado de Sidorenko, quanto o resultado de Soulis, Bountis e Dvorak, foram confirmados nesta dissertação, utilizando-se dois programas elaborados por Marcelo Farias Caetano e Manuel Valentim de Pera Garcia. No primeiro caso (resultado de sidorenko) foi feito um programa que calculava os índices de estabilidade (ver 4.3) para uma certa amplitude dada, usando como parâmetro apenas a amplitude $a$ do movimento, ou seja, $q_{3}\left(t_{0}\right)$. No segundo caso, foi feito um programa que imprimia o espaço de configuração da infinitesimal, usando como parâmetros, tamanho do passo (foi testado alguns passos no intervalo $\left[10^{-4}, 10^{-2}\right]$ ), a quantidade de passos, e as seis condições iniciais da infinitesimal (massa $m_{3}$ ), são elas: três momentos, $p_{1}\left(t_{0}\right)=0, p_{2}\left(t_{0}\right)=0, p_{3}\left(t_{0}\right)=0$, e três posições, $q_{1}\left(t_{0}\right) \in\left[-10^{-4}, 10^{-4}\right], q_{2}\left(t_{0}\right) \in\left[-10^{-4}, 10^{-4}\right], q_{3}\left(t_{0}\right)=a \in(5,13)$.

\subsection{Generalidades do problema}

Consideremos o problema dos três corpos restrito tridimensional e circular, com as primárias tendo massas iguais, digamos $m_{1}=m_{2}=m$. Utilizaremos as coordenadas rotacionais ou sinódicas. Portanto as coordenadas da infinitesimal no referencial sinódico serão usadas como coordenadas generalizadas.

$$
q_{1}=x_{1}, q_{2}=x_{2}, q_{3}=x_{3} .
$$

A função Hamiltoniana, em coordenadas sinódicas, adimensionais, pode ser dada por

$$
\mathcal{H}=\frac{1}{2}\left(p_{1}^{2}+p_{2}^{2}+p_{3}^{2}\right)+p_{1} q_{2}-p_{2} q_{1}-\frac{1}{2}\left(\frac{1}{r_{1}}+\frac{1}{r_{2}}\right) .
$$

Aqui $r_{1}$ e $r_{2}$ denotam as distâncias entre a infinitesimal e as primárias correspondentes ao sub-índice, enquanto que $p_{i}^{\prime} s$ são os momentos conjugados a $q_{i}$.

As equações Hamiltonianas do problema se reduzem a:

$$
\begin{aligned}
& \dot{q}_{1}=p_{1}+q_{2} \\
& \dot{q}_{2}=p_{2}-q_{1} \\
& \dot{q}_{3}=p_{3} \\
& \dot{p}_{1}=p_{2}-\frac{1}{2}\left(\frac{q_{1}-1 / 2}{r_{1}^{3}}+\frac{q_{1}+1 / 2}{r_{2}^{3}}\right) \\
& \dot{p}_{2}=-p_{1}-\frac{q_{2}}{2}\left(\frac{1}{r_{1}^{3}}+\frac{1}{r_{2}^{3}}\right) \\
& \dot{p}_{3}=-\frac{q_{3}}{2}\left(\frac{1}{r_{1}^{3}}+\frac{1}{r_{2}^{3}}\right)
\end{aligned}
$$

O espaço de fase $\nu=\{(p, q)\}$ possui uma subvariedade invariante pelo fluxo, $\tilde{\nu}=\{(p, q) \in$ $\mathbb{M}^{6}$ tal que $\left.p_{1}=p_{2}=q_{1}=q_{2}=0\right\}$. As trajetórias pertencentes a esta subvariedade correspondem aos movimentos verticais, com a infinitesimal sempre percorrendo o eixo $O x_{3}$. Consequentemente, esse movimento vertical é governado pelo sistema de um grau de liberdade com Hamiltoniano

$$
\tilde{\mathcal{H}}=\frac{p_{3}^{2}}{2}-\frac{1}{\sqrt{q_{3}^{2}+\frac{1}{4}}} .
$$

O retrato de fase do sistema (4.2) é dado na figura (4.1). É interessante perceber que as separatrizes (trajetórias que separam as hiperbólicas das periódicas), que correspondem aos movimentos 
parabólicos de $m_{3}$, se "interseptam"no infinito. As soluções periódicas associadas ao sistema com Hamiltoniano $\tilde{\mathcal{H}}$ formam uma família a um parâmetro

$$
p_{3}(t, a), q_{3}(t, a),
$$

onde temos a liberdade de escolher o parâmetro a como sendo ou a amplitude do movimento periódico, que pode ser calculado como $a=\max _{t \in \mathbb{R}}\left|q_{3}\right|$, ou o valor absoluto de $p_{3}$ quando $m_{3}$ passa pelo baricentro, ou o valor da energia $\tilde{\mathcal{H}}$ do movimento periódico. Aqui, por conveniência, usaremos a como a amplitude do movimento, ou seja, suporemos que

$$
p_{3}(0, a)=0, q_{3}(0, a)=a .
$$

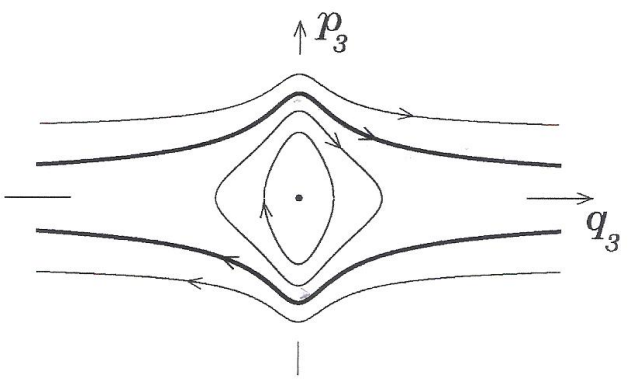

Figura 4.1: Retrato de fase na variedade $\tilde{\nu}$. Linha mais escura é a separatriz do espaço $(\tilde{\mathcal{H}}=0)$.

Existe uma expressão explícita de (4.3) em termos das integrais elípticas de Jacobi (Belbruno, Llibre e Ollé, [BLO94]) dada pelo seguinte teorema

Teorema 2. A solução do problema de Sitnikov circular pode ser escrita como:

$$
\begin{gathered}
r=\frac{1 / 2}{1-\frac{2+h}{2} s n^{2}(s / \sqrt{2}, k)}, \text { para }|h|<2, \\
r=\frac{1 / 2}{1-2 s n^{2}\left(k s / \sqrt{2}, k^{-1}\right)}, \text { para }|h|>2, \\
3-\frac{4(1+\sqrt{(2 r-1) r})}{2 r+1}=\exp (-\sqrt{2} s), \text { para } h=2,
\end{gathered}
$$

onde $r^{2}=q_{3}^{2}+1 / 4, k=\frac{1}{2}(2+h)^{1 / 2}, t=\int r^{2} d s$ e sn $(m, n)$ é a função elíptica de Jacobi.

Seja

$$
u(y, k)=u=\int_{0}^{y}\left[\left(1-t^{2}\right)\left(1-k^{2} t^{2}\right)\right]^{-1 / 2} d t
$$

uma integral elíptica. A função elíptica de Jacobi, $\operatorname{sn}(u, k)$, nada mais é do que a inversão da integral anterior, ou seja,

$$
\operatorname{sn}(u, k)=y .
$$

Para entender melhor sobre integrais elípticas ver [BF71].

Como não utilizaremos a solução explícita, não demonstraremos esse teorema. Sabendo a solução podemos também chegar em a fórmula fechada para o período das soluções de (4.3), dada pelo

Teorema 3. O período T para solução do problema de Sitnikov circular (4.3) para $-2<h<0$ pode ser escrito como

$$
T=\frac{\sqrt{2}}{1-2 k^{2}}\left\{E(k)+\frac{\pi}{2 \sqrt{2\left(1-2 k^{2}\right)}}\left[1-\Lambda_{0}\left(\arcsin \sqrt{\frac{1-2 k^{2}}{1-k^{2}}}, k\right)\right]\right\}
$$


onde $E(k)=\int_{0}^{\pi / 2}\left(1-k^{2} \sin ^{2} \theta\right)^{1 / 2} d \theta$ é a integral elíptica completa de segunda espécie, $k$ é como no teorema anterior e $\Lambda_{0}(m, n)$ é a função Lambda de Heuman (ver referência).

Sejam

$$
E(\phi, k)=\int_{0}^{\phi}\left(1-k^{2} \sin ^{2} \theta\right)^{1 / 2} d \theta
$$

e

$$
F(\phi, k)=\int_{0}^{\phi}\left(1-k^{2} \sin ^{2} \theta\right)^{-1 / 2} d \theta .
$$

Seja $k^{\prime}=\left(1-k^{2}\right)^{1 / 2}, E(k)=E(\pi / 2, k)$ e $F(k)=F(\pi / 2, k)$, então a função Lambda de Heuman é dada por

$$
\Lambda_{0}(\phi, k)=\frac{2}{\pi}\left(E(k) F\left(\phi, k^{\prime}\right)+F(k) E\left(\phi, k^{\prime}\right)-F(k) F\left(\phi, k^{\prime}\right)\right) .
$$

Para maiores detalhes sobre as funções Lambda Heuman e elíptica de Jacobi, conferir Byrd, [BF71].

Vale lembrar que usaremos o período dado anteriormente como parâmetros no algoritmo 1 (ver apêndice B), em que calculamos os valores de índice de estabilidade.

No teorema anterior $T$ depende implicitamente de $a$, pois a energia $h$ é uma função de $a$, dada por

$$
h=-\frac{1}{\sqrt{a^{2}+\frac{1}{4}}} .
$$

Algumas das propriedades do período são dadas abaixo:

Teorema 4. O período T satisfaz

- $\lim _{h \rightarrow-2} T(h)=\pi / \sqrt{2}$;

- $\lim _{h \rightarrow 0} T(h)=\infty$;

- $d T / d h>0 \forall h \in(-2,0)$;

- $\lim _{h \rightarrow-2} d T / d h=\pi(1+4 \sqrt{2}) / 16$

- $\lim _{h \rightarrow 0} d T / d h=\infty$.

Para movimentos com amplitudes grandes $(a>>1)$ a seguinte fórmula assintótica pode ser usada para aproximar o período:

$$
T \approx \sqrt{2} \pi a^{3 / 2} .
$$

Como foi mencionado antes as separatrizes $S^{ \pm}=\left\{\left(p_{3}^{ \pm}(t), q_{3}^{ \pm}(t)\right), t \in \mathbb{R}\right\}$, representa os movimentos parabólicos (escape), e pode ser interpretado como um limite formal para $a \rightarrow \infty$, dos movimentos periódicos, e obedecem a seguinte lei

$$
q_{3}^{ \pm}(t) \approx \pm\left(\frac{3}{\sqrt{2}}\right)^{2 / 3} t^{2 / 3}
$$

\subsection{Aproximação da matriz de monodromia}

\subsubsection{Propriedades do sistema linear}

Nosso intuito é analisar a estabilidade do movimento vertical, estabilidade essa com respeito a perturbações horizontais, nas quais a massa infinitesimal é deslocada do eixo $x_{3}$, para isso utilizaremos bastante o fato que para amplitudes grandes, o problema restrito pode ser aproximado por um problema de atração de uma massa por dois pontos fixos, [Lan81]. Vale mencionar que se 
perturbarmos "suavemente"a posição na direção do eixo $x_{3}$ em nada mudamos o movimento da infinitesimal, ou seja, o movimento continua periódico para qualquer perturbação "pequena"nessa direção. Utilizaremos as soluções da forma mostrada por Belbruno, [BLO94], (2), com período dado pelo teorema (3). Ou seja, fixando uma solução da forma do teorema (2), desta forma, estaremos também fixando o período e utilizando a equação variacional de Poincaré, [vide capítulo (2.3)], aplicada à essa solução, anaisaremos o comportamento das variáveis $p_{1}, p_{2}, q_{1}, q_{2}$, no movimento perturbado descrito pelo sistema Hamiltoniano linear com coeficientes periódicos,

$$
J \frac{d z}{d t}=H(t) z
$$

em que, $z=\left(p_{1}, p_{2}, q_{1}, q_{2}\right)^{T}$ e

$$
H(t)=\left(\begin{array}{cccc}
1 & 0 & 0 & 1 \\
0 & 1 & -1 & 0 \\
0 & -1 & \left(\frac{1}{D^{3}}-\frac{3}{4 D^{5}}\right) & 0 \\
1 & 0 & 0 & \left(\frac{1}{D^{3}}\right)
\end{array}\right)
$$

A função $D(t, a)=\left(q_{3}^{2}(t, a)+\frac{1}{4}\right)^{1 / 2}$ depende periodicamente do tempo, com período $T_{*}=T / 2$, em que $T$ denota o período do movimento vertical fixado (teorema 2) cuja a estabilidade estamos estudando.

Como é conhecido, o problema de Sitnikov circular admite vários tipos de simetrias. A consequência dessas simetrias é a seguinte propriedade da equação variacional (4.7): se $z(t)$ é uma solução de (4.7), então essa equação admite a solução

$$
\tilde{z}(t)=Q z(-t)
$$

onde $Q$ é a matriz diagonal $4 \times 4$ dada por $Q=\operatorname{diag}(1,-1,-1,1)$.

De acordo com a teoria de Floquet, [vide seção (2.3)], para se obter conclusões sobre estabilidade ou instabilidade das soluções de (4.7) podemos analisar as propriedades espectrais da matriz de monodromia $M=W\left(T_{*}, 0\right)$, onde $W\left(t, t^{\prime}\right)$ é a matriz fundamental correspondente ao sistema (4.7) (isto é, a matriz solução de (4.7) com condição inicial $\left.W\left(t^{\prime}, t^{\prime}\right)=I_{4}\right)$.

A matriz fundamental correspondente ao sistema linear Hamiltoniano (4.7) é simplética, portanto valem as seguintes propridades:

1. $W\left(t, t_{1}\right)=W\left(t, t_{2}\right) W\left(t_{2}, t_{1}\right)$;

2. $W\left(t+T_{*}, t^{\prime}+T_{*}\right)=W\left(t, t^{\prime}\right)$;

3. $W(0,-t)=Q W^{-1}(t, 0) Q=-Q J W^{T}(t, 0) J Q$,

desde que os argumentos estejam no domínio.

Usando as relações anteriores podemos obter

$$
\begin{gathered}
M=W\left(T_{*}, T_{*} / 2\right) W\left(T_{*} / 2,0\right)=W\left(0,-T_{*} / 2\right) W\left(T_{*} / 2,0\right)=Q W^{-1}\left(\frac{T_{*}}{2}, 0\right) Q W\left(\frac{T_{*}}{2}, 0\right) \\
=-Q J W^{T}\left(\frac{T_{*}}{2}, 0\right) J Q W\left(\frac{T_{*}}{2}, 0\right) .
\end{gathered}
$$

\subsubsection{Expressão aproximada da matriz de monodromia}

Nesta seção será apresentada uma aproximação para a expressão da matriz de monodromia. Conhecendo essa aproximação, pretendemos estudar numericamente a estabilidade e instabilidade, o encurtamento dos intervalos de estabilidade e instabilidade com o crescimento do parâmetro $a$, de uma solução periódica fixada. 
Como suporemos que a amplitude $a$ do movimento é grande ( $>546)$, podemos fixar uma quantidade auxiliar $d$ tal que

$$
1<<d<<a .
$$

Uma observação importante em se fazer é sobre essa quantidade auxiliar $d$. Como podemos ver pelo gráfico da energia pela amplitude (figura 4.2) quanto maior nossa amplitude $a$, mais próximo estaremos da separatriz, ou seja, quanto maior nosso parâmetro inicial do movimento periódico, mais próximo do parabólico estaremos. Intuitivamente podemos pensar que a melhor forma de aproximar um movimento com amplitude alta, é fazer as devidas aproximações com relação a solução parabólica. Este pensamento é correto, até certa amplitude $a^{\prime}<a$. Para fixarmos a idéia, vejamos o retrato de fase, (4.1), do sistema não perturbado. Dada uma certa "tolerância"de aproximação, ou seja, o quão próximo da separatriz nossa solução fixada está para podermos dizer que o movimento parabólico é uma boa aproximação do periódico, queremos saber até que momento nossa solução fixada ainda estará na região de tolerância, isto porque, nossa solução em algum momento terá que cruzar o eixo $O q$, portanto em algum momento ela sai da região de tolerância. Como o momento em que a solução fixada corta o eixo $O q$ é quando $q=a$ certamente a solução se afastará da região em algum momento em que a distância da infinitesimal à origem for menor que a amplitude dada. Esta distância $a^{\prime}$ é que chamaremos de $d$.

Para começar escreveremos a matriz de monodromia, $M=W\left(T_{*}, 0\right)$ como o produto de três matrizes fundamentais:

$$
M=W\left(T_{*}, t_{d}^{-}\right) W\left(t_{d}^{-}, t_{d}^{+}\right) W\left(t_{d}^{+}, 0\right),
$$

em que $t_{d}^{+} \in\left(0, T_{*} / 2\right)$ é o instante em que a infinitesimal, no movimento periódico representado por (4.3), está a uma distância $d$ da origem com velocidade positiva (ou seja, está se distanciando do baricentro), e $t_{d}^{-}=T_{*}-t_{d}^{+}$é o instante em que ela, no mesmo movimento periódico, está a uma distância $d$ do baricentro, mas com velocidade negativa (ou seja, está se aproximando da origem).

Motivados por (4.9) queremos aproximar uma solução em um certo intervalo de tempo, neste caso, meio período de um certo movimento periódico, por três funções, uma para cada um dos intervalos de tempo $\left(0, t_{d}^{+}\right),\left(t_{d}^{+}, t_{d}^{-}\right),\left(t_{d}^{-}, T_{*}\right)$, em que no final da construção essas três funções "colem bem"de forma que tenhamos a nossa solução aproximada.

- Expressão aproximada para a matriz $W\left(t_{d}^{+}, 0\right)$.

Se a condição (4.8) for satisfeita para uma trajetória $X\left(t, t_{0}, q_{0}\right)$ contida na subvariedade $\tilde{\nu}$ com $t \in I_{+}=\left[0, t_{d}^{+}\right]$, temos então, que a mesma está localizada em uma vizinhança "pequena"da separatriz $S^{+}$, pois ao vermos o gráfico (4.2) percebemos que quanto maior a amplitude inicial, ou seja, a energia inicial, mais próximo estaremos da assíntota que está no eixo $0 y$. Dentro deste intervalo a diferença entre $q_{3}(t, a)$ e $q_{3}^{+}(t)$ pode ser tomada arbitrariamente pequena. Negligenciando essa diferença, substituímos $q_{3}(t, a)$ em $(4.7)$ por $q_{3}^{+}(t)$, como os sistemas lineares $\left(H(t), H_{+}(t)\right)$ são próximos a matriz fundamental, $W_{+}(t, 0)$, do novo sistema será próxima da matriz fundamental do sistema antigo, ou seja, $W_{+}(t, 0)$, nos dá uma boa aproximação de $W(t, 0)$, no intervalo $I_{+}$.

O comportamento de $W_{+}(t, 0)$ no limite para $t \rightarrow \infty$ é descrito, conforme será mostrado a seguir, pela seguinte fórmula assintótica:

$$
W_{+}(t, 0) \approx R(t) \Lambda\left(q_{3}^{+}(t)\right) U,
$$

em que

$$
R(t)=\left(\begin{array}{cccc}
\cos t & \sin t & 0 & 0 \\
-\sin t & \cos t & 0 & 0 \\
0 & 0 & \cos t & \sin t \\
0 & 0 & -\sin t & \cos t
\end{array}\right)
$$




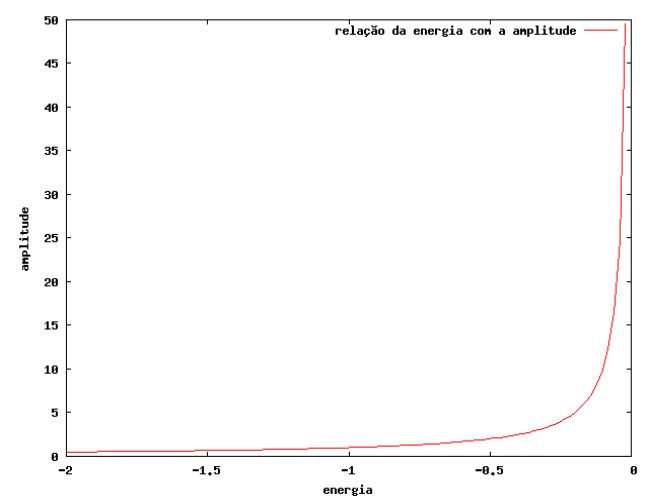

Figura 4.2: Gráfico da função (4.4).

$$
\begin{gathered}
\Lambda(q)=\left(\begin{array}{cccc}
\frac{1}{q} & 0 & -\sqrt{\frac{2}{q}} & 0 \\
0 & \frac{1}{q} & 0 & -\sqrt{\frac{2}{q}} \\
\sqrt{2 q} & 0 & -q & 0 \\
0 & \sqrt{2 q} & 0 & -q
\end{array}\right), \\
U=\left(\begin{array}{cccc}
0,3248 \ldots & 0,1020 \ldots & -0,4664 \ldots & 0,2228 \ldots \\
0,1302 \ldots & 0,1189 \ldots & 0,5296 \ldots & -2,0211 \ldots \\
1,1175 \ldots & 0,1718 \ldots & 1,4408 \ldots & 0,4791 \ldots \\
0,2113 \ldots & 0,6404 \ldots & 0,9414 \ldots & -2,5646 \ldots
\end{array}\right) .
\end{gathered}
$$

Como veremos mais para frente $U$ é obtida assintoticamente, devido a liberdade que podemos escolher $d$. Portanto é uma amtriz constante, com valores obtidos numericamente.

Provaremos agora a fórmula (4.10). Tomamos $L>>1$ e escrevemos $W_{+}(t, 0)$ como

$$
W_{+}(t, 0)=W_{+}\left(t, t_{L}\right) W_{+}\left(t_{L}, 0\right)
$$

onde $t_{L}$ é o tempo em que a infinitesimal está a uma distância $L$ da origem, na trajetória do movimento parabólico $q_{3}=q_{3}^{+}(t)$.

No próximo passo, modificaremos o sistema (4.7) para achar uma expressão aproximada para $W_{+}\left(t, t_{L}\right)$, para $t>t_{L}$. Como $t>t_{L}$ a terceira massa está suficientemente longe das primárias, é natural substituirmos $D$ por $q_{3}^{+}(t)$ no sistema (4.7) e negligenciar o termo pequeno $\frac{3}{4 D^{5}}$. Portanto o sistema (4.7) fica da seguinte maneira

$$
J \frac{d z}{d t}=\bar{H}\left(q_{3}^{+}(t)\right) z
$$

com

$$
\bar{H}(q)=\left(\begin{array}{cccc}
1 & 0 & 0 & 1 \\
0 & 1 & -1 & 0 \\
0 & -1 & \frac{1}{q^{3}} & 0 \\
1 & 0 & 0 & \frac{1}{q^{3}}
\end{array}\right)
$$

Agora vale a pena mencionar uma seguinte observação. Considere o movimento parabólico retilinear de um ponto material em um campo de atração central. Usando as unidades próprias, a distância entre o centro e o ponto material varia de acordo com a equação

$$
q(t)=\left(\frac{3}{\sqrt{2}}\right)^{2 / 3} t^{2 / 3}
$$

Portanto, para $L$ grande podemos usar a fórmula assintótica (4.6) no sistema (4.12). Então, 
se compararmos as equações (4.12) usando a fórmula assintótica para $q_{3}^{+}(t)$ e a as equações do movimento retilinear de um ponto material no campo de atração central linearizado em torno da solução (4.13), percebemos que as equações são as mesmas. Assim, ao resolvermos a equação de atração central linearizada em torno da solução (4.13), teremos uma aproximação para $W_{+}\left(t, t_{L}\right)$.

Considerando essa observação, podemos achar as soluções do problema linearizado acima. Primeiramente, usaremos a seguinte mudança de variável

$$
z=\left(p_{1}, p_{2}, q_{1}, q_{2}\right)^{T} \mapsto \bar{z}=\left(\bar{p}_{1}, \bar{p}_{2}, \bar{q}_{1}, \bar{q}_{2}\right)^{T},
$$

onde

$$
\bar{z}=R\left(t_{L}-t\right) z .
$$

Essa mudança de variável transforma referencial sinódico $(z)$ em referencial fixo $(\bar{z})$. Usando essa mudança de variável simplificaremos o problema, pois dividiremos as equações do movimento em dois subsistemas independentes dados por

$$
\frac{d \bar{p}_{i}}{d t}=-\frac{\bar{q}_{i}}{\bar{q}^{3}}, \quad \frac{d \bar{q}_{i}}{d t}=\bar{p}_{i}, i=1,2 .
$$

Usando técnicas habituais da teoria de equaçõs diferenciais, não é difícil achar soluções parciais para esse sistema. Como se pode verificar, algumas dessas soluções são

$$
\begin{aligned}
& \bar{p}_{1}=\dot{\bar{q}}, \quad \bar{q}_{1}=\bar{q}, \bar{p}_{2}=0, \quad \bar{q}_{2}=0, \\
& \bar{p}_{2}=\dot{\bar{q}}, \bar{q}_{2}=\bar{q}, \bar{p}_{1}=0, \quad \bar{q}_{1}=0, \\
& \bar{p}_{1}=\frac{1}{\bar{q}}, \bar{q}_{1}=\bar{q} \dot{\bar{q}}, \bar{p}_{2}=0, \quad \bar{q}_{2}=0 \text {, } \\
& \bar{p}_{2}=\frac{1}{\bar{q}}, \bar{q}_{2}=\bar{q} \dot{\bar{q}}, \bar{p}_{1}=0, \quad \bar{q}_{1}=0,
\end{aligned}
$$

e usando o fato de que a solução ao longo da qual foi linearizada é a solução parabólica, podemos o seguinte fato

$$
H=\frac{\|p\|^{2}}{2}-\frac{1}{\|q\|},
$$

para achar $\dot{\bar{q}}$, já que o hamiltoniano do movimento parabólico é nulo. Dessa forma, $\dot{\bar{q}}=\sqrt{\frac{2}{\bar{q}}}$.

Sabendo as soluções acima, podemos escrever a matriz fundamental do sistema (4.12) para $q$ dado por (4.13):

$$
\bar{W}\left(t, t_{L}\right)=\Lambda(\bar{q}(t)) \Lambda^{-1}\left(\bar{q}\left(t_{L}\right)\right) .
$$

Portanto a expressão aproximada de $\bar{W}_{+}\left(t, t_{L}\right)$ é dado por

$$
\bar{W}_{+}\left(t, t_{L}\right)=\Lambda\left(q_{3}^{+}(t)\right) \Lambda^{-1}(L) \approx \Lambda(\bar{q}(t)) \Lambda^{-1}\left(\bar{q}\left(t_{L}\right)\right) .
$$

Assim, retornando às variáveis iniciais, temos

$$
W_{+}\left(t, t_{L}\right)=R\left(t-t_{L}\right) \bar{W}_{+}\left(t, t_{L}\right) .
$$

Substituindo (4.15) em (4.11) obtemos a expressão aproximada para a matriz fundamental $W_{+}(t, 0)$ como o produto de três matrizes, e apenas uma depende explicitamente do tempo,

$$
W_{+}(t, 0) \approx R\left(t-t_{L}\right) \Lambda\left(q_{3}^{+}(t)\right) \Lambda^{-1}(L) W_{+}\left(t_{L}, 0\right),
$$

onde $R\left(t-t_{L}\right)=R(t) R\left(-t_{L}\right), \Lambda(q)$ e $\Lambda^{-1}(q)$ comutam com a matriz $R\left(-t_{L}\right)$. Logo temos a aproximação de $W_{+}(t, 0)$ dada por 


$$
W_{+}(t, 0) \approx R(t) \Lambda\left(q_{3}^{+}(t)\right) \bar{U}(L),
$$

onde $\bar{U}(L)=\Lambda^{-1}(L) R\left(-t_{L}\right) W_{+}\left(t_{L}, 0\right)$.

A fórmula (4.16) pode ser usada para calcularmos aproximação para os elementos da matriz $W_{+}(t, 0)$ para $t$ suficientemente grande. Assintoticamente seus valores não devem depender da escolha de $L$. Isto significa que o seguinte limite existe:

$$
U=\lim _{L \rightarrow \infty} \bar{U}(L) .
$$

Substituindo $U$ em (4.16) chegamos a (4.10).

Como $W_{+}(t, 0)$ foi introduzida como uma aproximação para $W(t, 0)$ no intervalo $I_{+}$, obtemos então que

$$
W\left(t_{d}, 0\right) \approx R\left(t_{d}\right) \Lambda(d) U
$$

- Expressão aproximada para a matriz $W\left(t_{d}^{-}, t_{d}^{+}\right)$

Para $t \in I=\left[t_{d}^{+}, t_{d}^{-}\right]$a massa $m_{3}$ está distante das primárias, portanto podemos considerar as primárias como uma grande massa com centro no baricentro, ou seja, reduziremos o problema ao caso dos dois corpos com campo de atração central, com centro localizado no baricentro das primárias. Nesse caso reduzimos a função Hamiltoniana de (4.1) para

$$
\mathcal{H}=\frac{\|p\|^{2}}{2}-\frac{1}{\|q\|}
$$

Para obtermos uma expressão aproximada para $W\left(t_{d}^{-}, t_{d}^{+}\right)$usando tal aproximação basta integrarmos o sistema

$$
J \frac{d z}{d t}=\bar{H}(\hat{q}(t, a)) z,
$$

onde $\hat{q}(t, a)$ descreve o movimento sobre um campo Newtoniano ao longo do segmento $[0, a]$ no eixo $O x_{3}$.

Suponha que a distância máxima $a$ (amplitude) da infinitesimal ao centro de atração seja atingida em $t=\frac{T_{*}}{2}$. Neste caso $q_{3}(t, a) \approx \hat{q}(t, a)$ em $t \in I$. Perceba que no caso aproximado (campo Newtoniano) a trajetória tende a uma singularidade (caso em que ocorre colisão no problema dos dois corpos) porém usaremos essa aproximação apenas num intervalo de tempo onde não ocorre essa singularidade.

Aqui vale lembrar a simetria do movimento, portanto, resolver o problema quando a infinitesimal parte de $d$ e chega em $a$ é equivalente a resolver o problema de quando ela parte de $a$ e chega em $d$. Pensando nisso usamos a mudança de variável

$$
z=\left(p_{1}, p_{2}, q_{1}, q_{2}\right)^{T} \mapsto \hat{z}=\left(\hat{p}_{1}, \hat{p}_{2}, \hat{q}_{1}, \hat{q}_{2}\right)^{T},
$$

onde

$$
\hat{z}=R\left(\frac{T_{*}}{2}-t\right) z
$$

para reescrevermos as equações (4.18) de uma forma mais simples (subsistemas independentes):

$$
\frac{d \hat{p}_{i}}{d t}=-\frac{\hat{q}_{i}}{\hat{q}^{3}}, \quad \frac{d \hat{q}_{i}}{d t}=\hat{p}_{i}, i=1,2 .
$$

Usando as mesmas técnicas que utilizamos para resolver (4.14), chegamos nas seguintes soluções parciais:

$$
\begin{aligned}
& \hat{p}_{1}=\dot{\hat{q}}, \hat{q}_{1}=\hat{q}, \quad \hat{p}_{2}=0, \quad \hat{q}_{2}=0, \\
& \hat{p}_{2}=\dot{\hat{q}}, \hat{q}_{2}=\hat{q}, \quad \hat{p}_{1}=0, \quad \hat{q}_{1}=0,
\end{aligned}
$$




$$
\begin{aligned}
& \hat{p}_{1}=\frac{1}{\hat{q}}-\frac{2}{a}, \hat{q}_{1}=\hat{q} \dot{\hat{q}}, \hat{p}_{2}=0, \quad \hat{q}_{2}=0, \\
& \hat{p}_{2}=\frac{1}{\hat{q}}-\frac{2}{a}, \quad \hat{q}_{2}=\hat{q} \dot{\hat{q}}, \hat{p}_{1}=0, \quad \hat{q}_{1}=0,
\end{aligned}
$$

ou, em forma matricial,

$$
\Sigma=\left(\begin{array}{cccc}
\frac{1}{\hat{q}}-\frac{2}{a} & 0 & \dot{\hat{q}} & 0 \\
0 & \frac{1}{\hat{q}}-\frac{2}{a} & 0 & \dot{\hat{q}} \\
\hat{q} \dot{\hat{q}} & 0 & \hat{q} & 0 \\
0 & \hat{q} \dot{\hat{q}} & 0 & \hat{q}
\end{array}\right)
$$

Para calcularmos $\dot{\hat{q}}$ nas soluções, de novo, basta usarmos a energia. Neste caso, em um campo Newtoniano, ao longo do segmento $[0, a]$, e usando o fato de o movimento começar em a (pela simetria do movimento), com velocidade inicial nula, a integral tem a seguinte forma

$$
\frac{\dot{\hat{q}}^{2}}{2}-\frac{1}{\hat{q}}=-\frac{1}{a}
$$

e consequentemente temos

$$
\dot{\hat{q}}(t)= \pm \sqrt{2\left(\frac{1}{\hat{q}(t)}-\frac{1}{a}\right)} .
$$

Levando isso em conta escrevemos a matriz fundamental do problema como sendo:

$$
\widehat{\mathcal{W}}\left(t, \frac{T_{*}}{2}\right)=\Sigma(\hat{q}(t)) \Sigma^{-1}\left(\hat{q}\left(T_{*} / 2\right)\right)=\left(\begin{array}{cccc}
2-\frac{a}{\hat{q}} & 0 & \frac{\dot{\hat{q}}}{a} & 0 \\
0 & 2-\frac{a}{\hat{q}} & 0 & \frac{\dot{q}}{a} \\
-a \hat{q} \hat{\hat{q}} & 0 & \frac{\hat{q}}{a} & 0 \\
0 & -a \hat{q} \hat{q} & 0 & \frac{\hat{q}}{a}
\end{array}\right) .
$$

Levando em conta a relação (4.19), escrevemos a matriz para o sistema (4.18)

$$
\mathcal{W}\left(t, T_{*} / 2\right)=R\left(t-\frac{T_{*}}{2}\right) \hat{\mathcal{W}}\left(t, T_{*} / 2\right) .
$$

Usando a expressão anterior encontramos

$$
\mathcal{W}\left(t_{d}^{-}, T_{*} / 2\right)=R\left(t_{d}^{-}-\frac{T_{*}}{2}\right) N(d, a),
$$

em que

$$
N(d, a)=\widehat{\mathcal{W}}\left(t_{d}^{-}, T_{*} / 2\right)=\left(\begin{array}{cccc}
2-\frac{a}{d} & 0 & -\frac{1}{a} \sqrt{2\left(\frac{1}{d}-\frac{1}{a}\right)} & 0 \\
0 & 2-\frac{a}{d} & 0 & -\frac{1}{a} \sqrt{2\left(\frac{1}{d}-\frac{1}{a}\right)} \\
d \sqrt{2 a\left(\frac{a}{d}-1\right)} & 0 & \frac{d}{a} & 0 \\
0 & d \sqrt{2 a\left(\frac{a}{d}-1\right)} & 0 & \frac{d}{a}
\end{array}\right) .
$$

Conhecendo a matriz $\mathcal{W}\left(t_{d}^{-}, T_{*} / 2\right)$ e usando as propriedades das soluções fundamentais concluímos que

$$
\mathcal{W}\left(T_{*} / 2, t_{d}^{+}\right)=Q \mathcal{W}^{-1}\left(t_{d}^{-}, T_{*} / 2\right) .
$$

Tendo isso, podemos agora calcular a aproximação para a matriz de $W\left(t_{d}^{-}, t_{d}^{+}\right)$,

$$
W\left(t_{d}^{-}, t_{d}^{+}\right) \approx \mathcal{W}\left(t_{d}^{-}, t_{d}^{+}\right)=\mathcal{W}\left(t_{d}^{-}, T_{*} / 2\right) \mathcal{W}\left(T_{*} / 2, t_{d}^{+}\right)
$$




$$
=\mathcal{W}\left(t_{d}^{-}, T_{*} / 2\right) Q \mathcal{W}^{-1}\left(t_{d}^{-}, T_{*} / 2\right) Q=R\left(t_{d}^{-}-t_{d}^{+}\right) N(d, a) Q N^{-1}(d, a) Q .
$$

A última igualdade se verifica pelo seguinte fato:

$$
\begin{gathered}
\mathcal{W}\left(t_{d}^{-}, T_{*} / 2\right) Q \mathcal{W}^{-1}\left(t_{d}^{-}, T_{*} / 2\right) Q=R\left(t_{d}^{-}-T_{*} / 2\right) N(d, a) Q N^{-1}(d, a) R\left(T_{*} / 2-t_{d}^{-}\right) Q= \\
=R\left(t_{d}^{-}-T_{*} / 2\right) N(d, a) Q R\left(T_{*} / 2-t_{d}^{-}\right) N^{-1}(d, a) Q=R\left(t_{d}^{-}-T_{*} / 2\right) N(d, a) R\left(-T_{*} / 2+t_{d}^{-}\right) Q N^{-1}(d, a) Q= \\
=R\left(t_{d}^{-}-T_{*} / 2\right) R\left(-T_{*} / 2+t_{d}^{-}\right) N(d, a) Q N^{-1}(d, a) Q=R\left(-T_{*}+2 t_{d}^{-}\right) N(d, a) Q N^{-1}(d, a) Q= \\
=R\left(t_{d}^{-}-t_{d}^{+}\right) N(d, a) Q N^{-1}(d, a) Q .
\end{gathered}
$$

Por fim, usando o fato que $a>>d$ podemos obter a seguinte aproximação para

$$
\begin{gathered}
N(d, a) Q N^{-1}(d, a) Q= \\
=\left(\begin{array}{cccc}
\frac{4 d}{a}-3 & 0 & -2\left(\frac{2}{a}-\frac{1}{d}\right) \sqrt{2\left(\frac{1}{d}-\frac{1}{a}\right)} & 0 \\
0 & \frac{4 d}{a}-3 & 0 & -2\left(\frac{2}{a}-\frac{1}{d}\right) \sqrt{2\left(\frac{1}{d}-\frac{1}{a}\right)} \\
2 d^{2} \sqrt{2\left(\frac{1}{d}-\frac{1}{a}\right)} & 0 & -2 d\left(\frac{1}{d}-\frac{1}{a}\right)+\left(\frac{2 d}{a}-1\right) & 0 \\
0 & 2 d^{2} \sqrt{2\left(\frac{1}{d}-\frac{1}{a}\right)} & 0 & -2 d\left(\frac{1}{d}-\frac{1}{a}\right)+\left(\frac{2 d}{a}-1\right)
\end{array}\right) \\
\approx K(d)=\left(\begin{array}{cccc}
-3 & 0 & 2 \sqrt{2} d^{-3 / 2} & 0 \\
0 & -3 & 0 & 2 \sqrt{2} d^{-3 / 2} \\
2 \sqrt{2} d^{3 / 2} & 0 & -3 & 0 \\
0 & 2 \sqrt{2} d^{3 / 2} & 0 & -3
\end{array}\right) .
\end{gathered}
$$

- Expressão aproximada para a matriz $W\left(T_{*}, t_{d}^{-}\right)$.

Usando novamente as propriedades da matriz fundamental para um sistema Hamiltoniano temos:

$$
W\left(T_{*}, t_{d}^{-}\right)=W\left(0,-t_{d}^{+}\right)=Q W^{-1}\left(t_{d}^{+}, 0\right) Q .
$$

Portanto usando a fórmula (4.17) na equação anterior chegamos na fórmula desejável para aproximar a matriz $W\left(T_{*}, t_{d}^{-}\right)$.

Conhecendo todas essas aproximações, as usaremos para chegar na fórmula aproximada da matriz de monodromia, dependente de $a$ somente.

$$
M\left(T_{*}, 0\right)=W\left(T_{*}, t_{d}^{-}\right) W\left(t_{d}^{-}, t_{d}^{+}\right) W\left(t_{d}^{+}, 0\right) \approx M(a),
$$

em que

$$
M(a) \approx\left\{Q(\Lambda(d) U)^{-1} Q R\left(T_{*}-t_{d}^{-}\right)\right\}\left\{R\left(t_{d}^{-}-t_{d}^{+}\right) K(d)\right\}\left\{R\left(t_{d}^{+} \Lambda(d) U\right\} .\right.
$$

Simplificando (4.24) chegamos na seguinte fórmula

$$
M(a) \approx Q U^{-1} Q R\left(T_{*}(a)\right) U .
$$

Com essa expressão poderemos estudar as propriedades de estabilidade das soluções periódicas no caso $a>>1$.

\subsection{Resultados numéricos}

Nesta seção mostraremos alguns resultados numéricos referente ao problema de sitnikov. Primeiro utilizaremos um programa elaborado por Marcelo Farias Caetano e Manuel Valentim de Pera 
Garcia, que nos mostra o movimento da massa $m_{3}$ (espaço de configuração do problema), com valores de amplitudes baixas (até 13) no espaço e testaremos alguns dos valores nos intervalos propostos por Soulis, [SBD07], para regiões de instabilidade e estabilidade.

Na segunda parte utilizaremos a aproximação da matriz de monodromia para movimentos com amplitudes $a>546$, para estudar a estabilidade e instabilidade de movimentos periódicos. Para isso testamos um programa elaborado por Marcelo Farias Caetano e Manuel Valentim de Pera Garcia, que calcula os coeficientes de instabilidade $\left(b_{1}\right.$ e $\left.b_{2}\right)$ dependentes dos multiplicadores da matriz de monodromia. Caso esses parâmetros sejam reais e estejam no intervalo $I_{1}=(-1,1)$ o movimento é estável, caso contrário o movimento é instável. No caso dos parâmetros serem complexos, testamos seus módulos. Caso o módulo seja igual a 1 o movimento é estável, caso contrário instável.

\subsubsection{Resultados para $5<a<13$}

Neste parágrafo, mostraremos alguns resultados numéricos para amplitudes com valores pequenos $(5<a<13)$. Utilizamos um programa elaborado por Marcelo \& Manuel, que imprime o espaço de configuração da massa $m_{3}$ (ver algoritmo 2, apêndice B). Neste programa, pede-se a entrada de oito parâmetros: passo de integração para ser utilizado no método de integração utilizado (RungeKutta de quarta ordem), quantos passos devem ser calculados, três momentos iniciais $\left(p_{0}^{1}, p_{0}^{2}, p_{0}^{3}\right.$, ) e três posições iniciais $\left(q_{0}^{1}, q_{0}^{2}, q_{0}^{3},\right)$. Elaboramos o espaço de configuração para certos valores de entrada, para os momentos foram utilizados valores no intervalo $\left[-10^{-4}, 10^{-4}\right]$, para as posições $q_{0}^{1}$ e $q_{0}^{2}$, foram utilizados valores no intervalo $\left[-10^{-2}, 10^{-2}\right]$ e para $q_{0}^{3}=a$ usamos os valores propostos por Soulis, [SBD07], [ver tabela (4.1)].

Como podemos ver nas figuras (4.3) e (4.5), utilizamos valores de $a$ nos intervalos de estabilidades e nas figuras (4.4) e (4.6), utilizamos valores fora de algum intervalo de estabilidade.
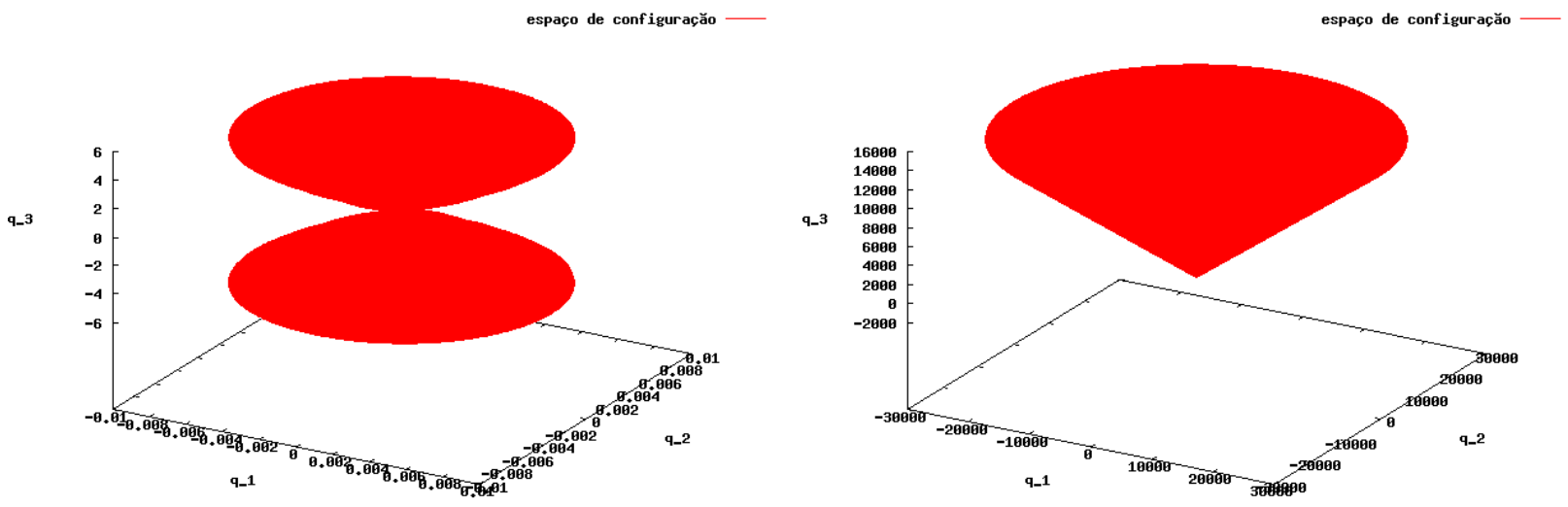

Figura 4.3: Trajetória realizada pela massa infinite- Figura 4.4: Trajetória realizada pela massa infinitesimal no espaço para a amplitude inicial de 5.0500. simal no espaço para a amplitude inicial de 5.0525.

O interessante em perceber, ao vermos a tabela (4.1), é o comportamento dos comprimentos dos intervalos de estabilidade e instabilidade, que parecem tender a um número positivo. Para explicar esse fenômeno, iremos introduzir a funções $\delta_{e s t}(a)$, que dá a relação entre a amplitude e o comprimento do intervalo de estabilidade. Essa função é proporcional a uma função $a^{-1 / 2}$, ou seja

$$
\begin{gathered}
\delta_{\text {est }}(a) \approx 0,3 a^{-1 / 2}, \\
\delta_{\text {inst }}(a) \approx 0,64 a^{-1 / 2},
\end{gathered}
$$

em que $k$ é uma constante e $a$ a amplitude.

Dessa função $\delta_{\text {est }}$ concluímos que o comportamente convergente de $\delta_{\text {est }}(a)$ é explicado pelo fato de que estamos perto do máximo da função, isto é, a função $\delta_{\text {est }}$ atinge seu máximo local próximo a $a=13$, depois ela é estritamente decrescente, sendo assim ao variarmos pouco a amplitude perto 


\begin{tabular}{|c|c|c|}
\hline Limite inferior & Limite superior & Comprimento do intervalo \\
\hline \hline 5,044 & 5,052 & 0,008 \\
5,453 & 5,470 & 0,017 \\
5,848 & 5,872 & 0,024 \\
6,230 & 6,259 & 0,029 \\
6,600 & 6,634 & 0,034 \\
6,961 & 6,999 & 0,037 \\
7,314 & 7,353 & 0,039 \\
7,657 & 7,699 & 0,042 \\
7,994 & 8,037 & 0,044 \\
8,323 & 8,368 & 0,045 \\
8,646 & 8,692 & 0,046 \\
8,965 & 9,010 & 0,045 \\
9,276 & 9,323 & 0,047 \\
9,583 & 9,630 & 0,047 \\
9,885 & 9,933 & 0,048 \\
10,182 & 10,231 & 0,049 \\
10,476 & 10,524 & 0,048 \\
10,765 & 10,814 & 0,049 \\
11,050 & 11,099 & 0,049 \\
11,332 & 11,381 & 0,049 \\
11,610 & 11,660 & 0,050 \\
11,885 & 11,935 & 0,050 \\
12,157 & 12,207 & 0,050 \\
12,425 & 12,475 & 0,050 \\
12,691 & 12,741 & 0,050 \\
\hline
\end{tabular}

Tabela 4.1: Intervalos de estabilidades para o parâmetro a do problema de Sitnikov, [SBD07]. Os limites inferior e superior são os bordos do intervalo de estabilidade. Fora desse intervalo o movimento é instável.
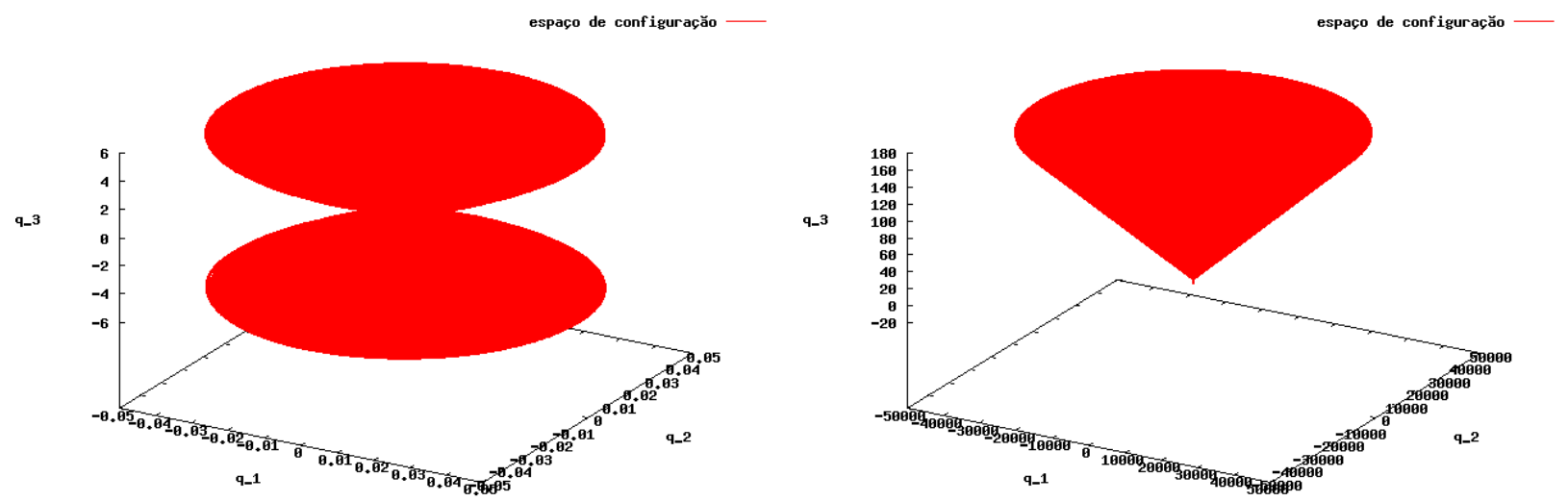

Figura 4.5: Trajetória realizada pela massa infinite- Figura 4.6: Trajetória realizada pela massa infinitesimal no espaço para a amplitude inicial de 5.4600. simal no espaço para a amplitude inicial de 5.4701.

do seu máximo o comprimento do intervalo, $\delta_{e s t}$, não alterará muito. Esse foi o erro cometido por Soulis, [SBD07], como o resultado foi obtido numericamente, aparentemente há a impressão de que o comprimento do intervalo tende a 0,05 quando $a$ tende ao infinito, o que, realmente não ocorre (como será mostrado no parágrafo seguinte, ao utilizarmos a expressão da matriz de monodromia aproximada, para valores grandes de $a$.) 


\subsubsection{Resultados para $a>546$}

O polinômio característico para o sistema (4.7)

$$
\operatorname{det}(M-\rho I)=0
$$

é simétrico, portanto podemos escrevê-lo da seguinte forma

$$
\rho^{4}-c_{1} \rho^{3}+c_{2} \rho^{2}-c_{1} \rho+1=0,
$$

onde $c_{1}=\operatorname{tr} M$, e $c_{2}=\sum_{j=1}^{3} \sum_{k=j+1}^{4}\left(m_{j j} m_{k k}-m_{j k} m_{k j}\right)$. Na equação anterior $m_{j k}$ são os elementos da matriz de Monodromia $M$.

Podemos reescrever o polinômio característico (4.26) como o produto

$$
\left(\rho^{2}-2 b_{1} \rho+1\right)\left(\rho^{2}-2 b_{2} \rho+1\right)=0 .
$$

Os coeficientes $b_{1}$ e $b_{2}$ em (4.27) são as raízes (reais ou complexas) da equação quadrática:

$$
4 x^{2}-2 c_{1} x+\left(c_{2}-2\right)=0 .
$$

Chamaremos $b_{1}$ e $b_{2}$ de índices de estabilidade (Bountis \& Papadakis ([BP09])).

Como podemos ver os índices de estabilidade podem ser escrito como

$$
\begin{aligned}
& b_{1}=\frac{2 c_{1}+\sqrt{4 c_{1}^{2}-16\left(c_{2}-2\right)}}{8}, \\
& b_{2}=\frac{2 c_{1}-\sqrt{4 c_{1}^{2}-16\left(c_{2}-2\right)}}{8},
\end{aligned}
$$

e os multiplicadores como

$$
\begin{aligned}
& \rho_{1,2}=b_{1} \pm \sqrt{b_{1}^{2}-1}, \\
& \rho_{3,4}=b_{2} \pm \sqrt{b_{2}^{2}-1} .
\end{aligned}
$$

Deste fato, utilizando a tabela (2.1), concluimos que o movimento vertical periódico é estável para todo $b_{i} \in I_{1}=(-1,1), i=1,2$, pois temos quatro multiplicadores diferentes com módulo igual a 1 . Caso $b_{i} \notin \bar{I}_{1}, i=1,2$, o movimento é claramente instável. Sobra o caso em que $b_{i} \in \partial I_{1}, i=1,2$, neste caso podemos ver que o movimento também é instável, pelo fato que temos pelo menos dois multiplicadores iguais e com módulo igual a 1.

No caso complexo $\left(b_{i} \in \mathbb{C}, i=1,2\right.$,), acontece um fenômeno interessante, analisando o módulo $\operatorname{dos} b_{i}$ 's numericamente e calculando os módulos dos multiplicadores em função desses índices de estabilidade, tem-se que se os módulos dos $b_{i}$ 's forem iguais a 1 os multiplicadores também teriam módulo 1 , então a primeira vista se os módulos dos $b_{i}$ 's fossem iguais a 1 o movimento seria estável, porém neste caso os multiplicadores não seriam simples, na verdade existiriam dois multiplicadores com multiplicidade algébrica dois (os valores dos multiplicadores foram calculados em função dos índices), portanto o movimento não seria estável segundo Lyapunov.

$\mathrm{Na}$ figura (4.7) apresentamos o comportamento dos índices de estabilidade $b_{1}$ e $b_{2}$ próximo da primeira aparição da "sela complexa". Percebemos que para alguns intervalos de $a$, não existe valores dos índices (pois nessa região eles são complexos), devido a forma dos gráficos nesse região, chamamos esse intervalo de "sela complexa", e nesse intervalo o movimento periódico é instável.

Tomando como base essa análise e utilizando a expressão aproximada da matriz de monodromia, foi verificada a existência de quatro intervalos, [Sid11], comprovada por Marcelo Farias Caetano e Manuel Valentim de Pera Garcia, quando variamos a amplitude, a, esses intervalos estão na seguinte ordem e tem respectivas propriedades:

1. Intervalo de instabilidade "largo": aqui os dois coeficientes de instabilidade são reais, mas um deles tem módulo diferente de 1 (instabilidade centro-sela). O comprimento assintótico 


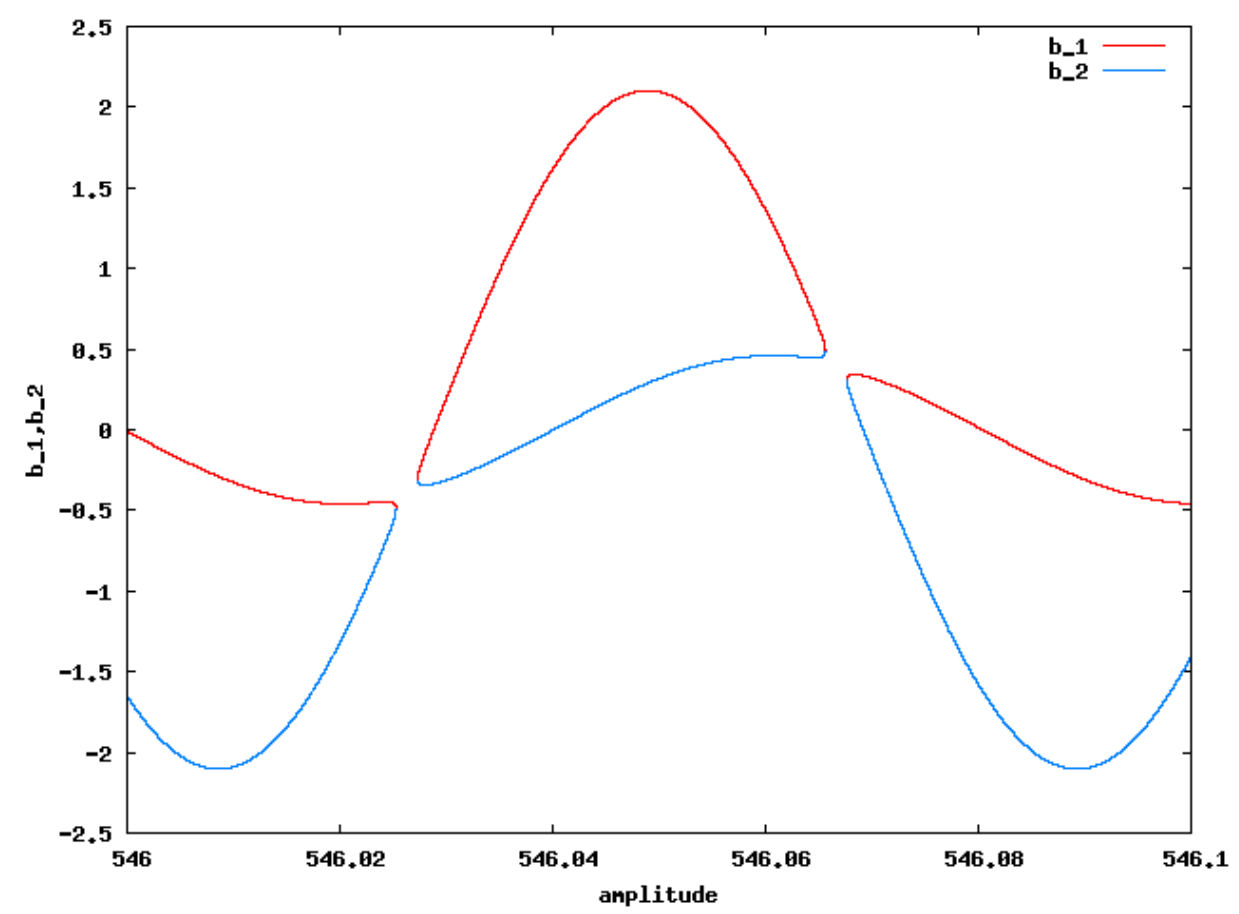

Figura 4.7: Comportamento dos indices de estabilidade na vizinhança de onde ocorre as primeiras "selas complexas"(região em branco no gráfico).

desse intervalo em termos da amplitude é dado por: $0,643544 a^{-1 / 2}$, enquanto a variação do semiperíodo é igual a 2.144392.

2. Intervalo de estabilidade "curto": aqui os dois coeficientes de instabilidade são reais, e estão em $I_{1}$. O comprimento assintótico desse intervalo em termos da amplitude é dado por: $0,068655 a^{-1 / 2}$, enquanto a variação do semiperíodo é igual a 0.228768 .

3. Intervalo de instabilidade "curto": aqui os dois coeficientes de instabilidade são complexos, com pelo menos um deles com módulo diferente de 1 (instabilidade de sela complexo). $\mathrm{O}$ comprimento assintótico desse intervalo em termos da amplitude é dado por: 0, $048166 a^{-1 / 2}$, enquanto a variação do semiperíodo é igual a 0,160497.

4. Intervalo de estabilidade "largo": aqui os dois coeficientes de instabilidade são reais, e, também, estão em $I_{1}$. O comprimento assintótico desse intervalo em termos da amplitude é dado por: $0,182445 a^{-1 / 2}$, enquanto a variação do semiperíodo é igual a 0.607936 .

A tabela (4.2) mostra alguns valores testados para a amplitude e seu respectivo intervalo de estabilidade ou instabilidade. Na figura (4.8) é mostrado a figura (4.7) ampliada na região tabelada.

Podemos construir numericamente um análogo, para $a>546$, da fórmula para o comprimento o intervalo de estabilidade em função a amplitude. Como podemos perceber de (4.25) os coeficientes da matriz de monodromia e, respectivamente, os coeficientes da equação característica são funções $2 \pi$-periódicas do semiperíodo, $T_{*}$, do movimento vertical. Usando a fórmula do período dado no teorema (3), não é difícil provar que em termos de $a$ os comprimentos dos intervalos de estabilidades e instabilidade decrescem proporcionalmente a $a^{-1 / 2}, a \rightarrow \infty$. Sendo que ao somarmos os dois intervalos de estabilidade (curto e longo) mais o intervalo de instabilidade curto nos leva a seguinte fórmula:

$$
\delta_{\text {est }}^{t r} \approx 0.299 a^{-1 / 2},
$$

chamado intervalo de estabilidade transiente, isto nada mais é do que a partição do antigo intervalo de estabilidade em três novos intervalos (como podemos ver $\delta_{e s t}^{t r} \approx \delta_{\text {est }}$ ). O que é uma ótima aproximação de acordo com os cálulos numéricos. 


\begin{tabular}{|c|c|c|}
\hline Amplitude & Estabilidade & b's \\
\hline \hline 546.020 & instável & $b_{2}=-1.319, b_{1}=-0.459$ \\
546.021 & instável & $b_{2}=-1.186, b_{1}=-0.459$ \\
546.022 & instável & $b_{2}=-1.047, b_{1}=-0.456$ \\
546.023 & estável & $b_{2}=-0.899, b_{1}=-0.452$ \\
546.024 & estável & $b_{2}=-0.742, b_{1}=-0.448$ \\
546.025 & estável & $b_{2}=-0.569, b_{1}=-0.454$ \\
546.026 & instável & $b_{i} \in \mathbb{C}$ \\
546.027 & instável & $b_{i} \in \mathbb{C}$ \\
546.028 & estável & $b_{2}=-0.344, b_{1}=-0.144$ \\
546.029 & estável & $b_{2}=-0.332, b_{1}=0.028$ \\
546.030 & estável & $b_{2}=-0.312, b_{1}=0.196$ \\
\hline
\end{tabular}

Tabela 4.2: Valores das amplitudes (adimensional) inicial e respectiva estabilidade do movimento periódico vertical em que ela se encontra e os valores dos indices de estabilidade.

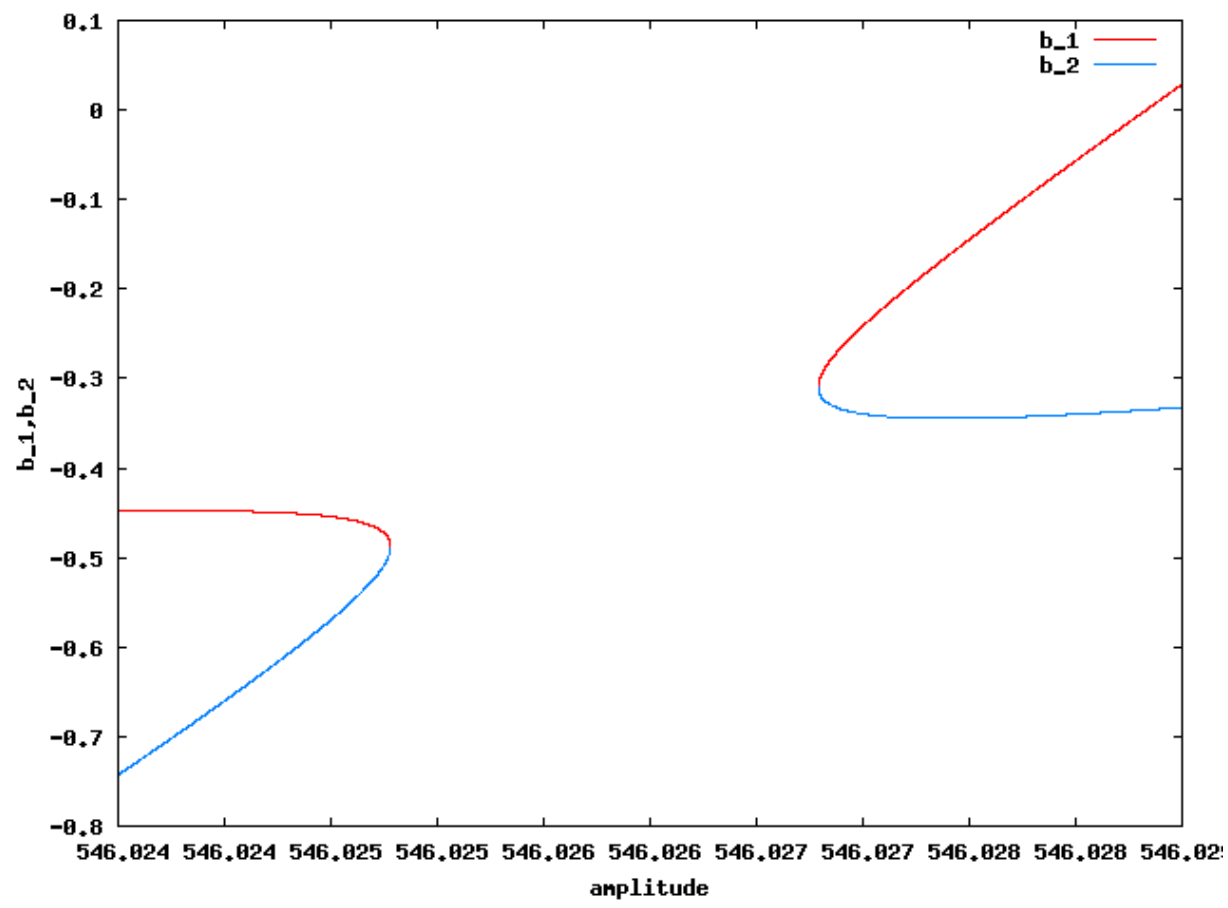

Figura 4.8: Comportamento dos indices de estabilidade na vizinhança de onde ocorre as primeiras "selas complexas"(região em branco no gráfico). 


\section{Capítulo 5}

\section{Conclusões}

Combinando análise assintótica com teoria de matrizes e análise numérica, pôde-se corrigir e estender alguns resultados antes conhecidos, relacionados ao problema de Sitnikov circular (ver [SBD07], [BP09], [PM88]), que dizem respeito a estabilidade do movimento vertical. Pela primeira vez foi apresentado a possibilidade da existência "sela complexa"na família do movimento vertical.

De acordo com a construção teórica da matriz de monodromia referente à essa família, é essencial que a trajetória periódica correspondente esteja, em algum intervalo de tempo, na vizinhança da separatriz do problema. Em geral é possível estudar localmente estes tipos de soluções (próximas da separatriz) introduzindo aplicações auxiliares. Porém, devido a peculiaridade da separatriz do problema de Sitnikov circular (solução ilimitada), estudou-se o problema usando ténicas de análise assintótica e computacional.

De fato, computacionalmente, foi possível confirmar (antes percebida por Sidorenko) a existência de "selas complexas"e concluir que o comprimento do intervalo de estabilidade, isto é, o comprimento do intervalo da amplitude inicial, no qual a trajetória é dependente e no mesmo continua limitada, não converge a 0,05 como proposto por Soulis et al. [SBD07], mas que de fato esse comprimento converge a zero com o crescimento da amplitude inicial.

\subsection{Considerações Finais}

Podemos pensar no mesmo problema da estabilidade no caso em que o problema de Sitnikov circular tenha quatro ou mais corpos. Neste caso, em contraste ao problema de três corpos, não há alternância estabilidade/instabilidade na família do movimento vertical. Em particular, usando técnicas similares às usadas no estudo da estabilidade do movimento vertical no problema dos 3 corpos, [Sid11], problema de estabilidade do movimento vertical para quatro corpos mostrou-se sempre instável para valores grandes de amplitudes. 
CONCLUSÕES 


\section{Apêndice A}

\section{Funções matriciais e logaritmo de matriz}

Para se entender melhor sistemas periódicos, precisamos de alguma informação sobre logaritmo de matrizes. Para provarmos que uma matriz simplética tem um logaritmo Hamiltoniano, usaremos teoria de funções analíticas de matrizes. Essa teoria não é largamente conhecida, portanto aqui teremos como objetivo dar uma breve introdução sobre o assunto.

\section{A.1 Funções matriciais}

\section{A.1.1 Funções matriciais holomórficas}

Seja $f(z)=\sum_{k=0}^{\infty} a_{k} z^{k}$ uma função na variável complexa $z$, convergente com raio de convergência $R$ com os $a_{k}^{\prime} s \in \mathbb{C}$, isto é, $f(z)$ é uma função analítica no disco complexo $|z|<R$.

Se substituirmos $z$ por $A$, matriz $n \times n$ na função acima, temos a função matricial, $f(A)$, definida pela série

$$
f(A)=\sum_{k=0}^{\infty} a_{k} A^{k} .
$$

Podemos dizer que essa série é convergente se todas as $n^{2}$ séries escalares dos elementos de $f(A)$ são convergente. Podemos também dizer que a série (A.1) é convergente se todos os autovalores da matriz $A$ estão no interior do disco de convergência de raio $R$ (mesmo disco de $f(z)$ ), ou seja $\left|\lambda_{i}\right|<R, i=1, \ldots, k \leq n$. A série diz-se divergente, no caso em que pelo menos um desses autovalores está fora do disco de convergência.

Usando qualquer norma de matriz $\|\bullet\|$, satisfazendo $\|I\|=1$ e $\|A B\| \leq\|A\|+\|B\|$, pode-se provar que para toda matriz $A$ tal que $\|A\|<R$, a série $f(A)$ é convergente (implicando que cada coeficiente da matriz é uma série convergente), ou seja, $f(A)$ tem mesmo raio de convergência de $f(z)$. A teoria de funções analíticas para matriz é parecida com a teoria de funções analíticas para uma variável complexa. Isso quer dizer que as séries

$$
\begin{gathered}
e^{A}=I+\sum_{k=1}^{\infty} \frac{1}{k !} A^{k} \\
\cos (A)=I+\sum_{k=1}^{\infty}(-1)^{k} \frac{1}{2 k !} A^{2 k}, \\
\sin (A)=I+\sum_{k=1}^{\infty}(-1)^{k-1} \frac{1}{(2 k-1) !} A^{2 k-1},
\end{gathered}
$$

são convergentes para qualquer matriz quadrada $A$. 
Similarmente, a série

$$
\log (A)=\log [I+(A-I)]=\log (A)=I+\sum_{k=1}^{\infty}(-1)^{k-1} \frac{1}{k}(A-I)^{k},
$$

é convergente para $|A-I|<1$.

E mais, valem as seguintes identidades

$$
\begin{gathered}
e^{i A}=\cos (A)+i \sin (A) \\
e^{A} e^{A}=e^{2 A}, \cos ^{2}(A)+\sin ^{2}(A)=I .
\end{gathered}
$$

Caso $A$ e $B$ comutem, também vale

$$
e^{A} e^{B}=e^{A+B}, \quad \sin (A+B)=\sin (A) \cos (B)+\sin (B) \cos (A) .
$$

A definição de $f(A)$, comporta-se normalmente bem com respeito a similaridades das transformações, ou seja,

$$
f\left(P^{-1} A P\right)=P^{-1} f(A) P,
$$

portanto, se $\lambda$ for autovalor de $A$ então $f(\lambda)$ será um autovalor de $f(A)$. Se $N$ for um bloco de Jordan então $f(N)$ tem forma triangular

$$
\begin{gathered}
\left(\begin{array}{ccccc}
\lambda & 1 & 0 & \ldots & 0 \\
0 & \lambda & 1 & \ldots & 0 \\
\vdots & \vdots & \ddots & \ddots & \vdots \\
0 & 0 & 0 & \ldots & \lambda
\end{array}\right) . \\
f(N)=\left(\begin{array}{ccccc}
f(\lambda) & f^{\prime}(\lambda) & \frac{f^{\prime \prime}(\lambda)}{2 !} & \ldots & \frac{f^{(k-1)}(\lambda)}{(k-1) !} \\
0 & f(\lambda) & \frac{f^{\prime \prime}(\lambda)}{2 !} & \ldots & \frac{f^{(k-2)}(\lambda)}{(k-2) !} \\
\vdots & \vdots & \ddots & \ddots & \vdots \\
0 & 0 & 0 & \ldots & f(\lambda)
\end{array}\right) .
\end{gathered}
$$

E vale a fórmula integral

$$
f(A)=\frac{1}{2 \pi i} \oint_{\Gamma}(\xi I-A)^{-1} f(\xi) d \xi,
$$

desde que o domínio de analiticidade de $f$ contenha o espectro de $A$ no interior e este fique na região delimitada por $\Gamma$, mas não intercepte $\Gamma$.

Em particular

$$
I=\frac{1}{2 \pi i} \oint_{\Gamma}(\xi I-A)^{-1} d \xi
$$

$$
A=\frac{1}{2 \pi i} \oint_{\Gamma}(\xi I-A)^{-1} \xi d \xi
$$

\section{A.1.2 Funções matriciais analíticas por partes}

Daremos aqui uma definição de funções matriciais, em termos de integrais de linha, que é frequentemente mais conveniente. Essa definição também nos leva a uma classe grande de funções. Seja $G$ a união de um número finito de conjuntos simplesmente conexos, dois a dois disjuntos no plano complexo (por exemplo, a união finita de discos disjuntos). Chamaremos esses subconjuntos de domínios e os denotaremos por $G_{l}$, e suponha que em seu interior $(\operatorname{int}(G))$ esteja definida uma função analítica $f(z)$. Note que não pedimos a continuação analítica dessa função de um domínio 
para o outro, portanto $f(z)$ não precisa definir uma função analítica no sentido de Weierstrass. Chamaremos essa $f(z)$ de função analítica por partes.

Como ilustração, temos o seguinte exemplo: seja $G$ a união de dois discos disjuntos $G_{1}$ e $G_{2}$. Defina a função $f(z)$ como

$$
f(z)=\left\{\begin{array}{l}
\sin (z), \text { se } z \in G_{1} \\
\cos (z), \text { se } z \in G_{2} .
\end{array}\right.
$$

Seja $\mathcal{M}_{G}$ o conjunto de todas as matrizes cujo espectro (conjunto de autovalores) esteja em $G$. Fixe uma matriz $A, n \times n$ desse conjunto. Envolva todos os autovalores de $A$, que estão em algum $G_{l}$, por uma curva $\Gamma_{l}$ (assumimos que a curva é fechada, suave por partes ou retificável e que a curva envolva cada autovalor $\lambda_{l}^{j}, 1 \leq j \leq n_{l}$ apenas uma vez) inteiramente contida no interior de $G_{l}$ (os autovalores não estão no bordo das curvas $\Gamma_{l}$ ). Definimos a função matricial (ou simplesmente matriz) pela fórmula de Cauchy

$$
f(A)=\frac{1}{2 \pi i} \sum_{j=1}^{q} \oint_{\Gamma_{j}}(\xi I-A)^{-1} f(\xi) d \xi,
$$

onde a orientação de $\Gamma_{j}$ é tomada positivo, isto é, $\Gamma_{j}$ é orientada no sentido anti-horário.

Usando cálculo de resíduos pode-se mostrar que as definições (A.3) e (A.1) coincidem, portanto, de agora em diante onde pedirmos função analítica por partes pode-se pensar que ela é realmente uma função holomorfa.

\section{A.1.3 Lema da composição de funções}

Definição. Seja $\left(z-\lambda_{k}\right)^{m_{k}}(k=1, \ldots, s)$ os divisores elementares da matriz A. Dizemos que as funçôes $f_{1}(z)$ e $f_{2}(z)$ analíticas nos autovalores $\lambda_{1}, \ldots, \lambda_{s}$ de $A$ coincidem no espectro de $A$ se

$$
f_{1}\left(\lambda_{k}\right)=f_{2}\left(\lambda_{k}\right), f_{1}^{\prime}\left(\lambda_{k}\right)=f_{2}^{\prime}\left(\lambda_{k}\right), \ldots, f_{1}^{m_{k}-1}\left(\lambda_{k}\right)=f_{2}^{m_{k}-1}\left(\lambda_{k}\right), k=1, \ldots, s .
$$

Aqui, $s$ é o número de autovalores distintos de $A$ e $m_{1}, \ldots, m_{k}$ suas respectivas multiplicidades algébricas.

Lema 1. Se $f_{1}(z)$ e $f_{2}(z)$ coincidem no espectro de $A$, então $f_{1}(A)=f_{2}(A)$

Portanto, usando este lema, dada uma matriz $A$, de ordem $n \times n$, e uma função $f(z)$, analítica por partes, podemos construir um polinômio $P(z)$ de grau no máximo $n-1$ tal que $f(A)=P(A)$.

Lema da composição de funções. Seja $\phi(z)$ uma função analítica nos complexos, definida em um conjunto $G$, o qual é uma união finita de dominios simplesmente conexos; denote $\phi(G)=H$. Seja $\psi(w)$ uma função analitica nos complexos, definida em $H$; portanto podemos definir uma função $f(z)$ analítica em $G$, por $f(z)=\psi[\phi(z)]$. Suponha que o espectro da matriz A esteja em $G$ e que o espectro de $B=f(A)$ esteja em $H$.

Então $f(A)=\psi(B)$. Ou seja, o resultado de calcular $B=\phi(A)$ e depois $\psi(B)=\psi[\phi(A)]$ é igual ao de calcular $f(A)=(\psi \circ \phi)(A)$.

Demonstração. Dada a matriz $A$ e a função anlítica $\phi(z)$, podemos construir um polinômio $\Phi(z)=$ $\sum_{k=0}^{q} \alpha_{k} z^{k}$ tal que $\phi(A)=\Phi(A)$. Para isso, usaremos as condições

$$
\phi\left(\lambda_{k}\right)=\Phi\left(\lambda_{k}\right), \ldots, \phi^{\left(l_{k}-1\right)}\left(\lambda_{k}\right)=\Phi^{\left(l_{k}-1\right)}\left(\lambda_{k}\right),
$$

onde $\lambda_{k}, k=1, \ldots, q$, são os autovalores distintos de $A$ e $l_{k}$ é a ordem da maior matriz de Jordan elementar correspondente ao autovalor $\lambda_{k}\left(l_{1}+\ldots+l_{q} \leq n\right)$. Fazemos a mesma construção para $\operatorname{achar} \Psi(w)$,

$$
\psi\left(\mu_{k}\right)=\Psi\left(\mu_{k}\right), \ldots, \psi^{\left(l_{k}-1\right)}\left(\mu_{k}\right)=\Psi^{\left(l_{k}-1\right)}\left(\mu_{k}\right),
$$

aqui $\mu_{k}=\phi\left(\lambda_{k}\right)(k=1, \ldots, q)$ são os autovalores de $B$. Queremos portanto provar que o polinômio $F(z)=\Psi[\Phi(z)]$ e a função $f(z)$ coincidam no espectro de $A$. 
De fato

$$
\begin{gathered}
F\left(\lambda_{k}\right)=\Psi\left[\Phi\left(\lambda_{k}\right)\right]=\Psi\left(\mu_{k}\right)=\psi\left(\mu_{k}\right)=f\left(\lambda_{k}\right), \\
F^{\prime}\left(\lambda_{k}\right)=\Psi^{\prime}\left[\mu_{k}\right] \Phi^{\prime}\left(\lambda_{k}\right)=\psi^{\prime}\left(\mu_{k}\right) \phi^{\prime}\left(\lambda_{k}\right)=f^{\prime}\left(\lambda_{k}\right), \\
\ldots \ldots \\
\ldots \ldots \\
F^{\left(l_{k}-1\right)}\left(\lambda_{k}\right)=f^{\left(l_{k}-1\right)}\left(\lambda_{k}\right) .
\end{gathered}
$$

Portanto $F(z)$ e $f(z)$ coincidem no espectro de $A$ e portanto $F(A)=f(A)$. Seja $C_{1}$ o resultado da conta por $\psi[\phi(A)]$, e $C_{2}$ o resultado direto da conta de $(\psi \circ \phi)(A)$, temos

$$
B=\phi(A)=\Phi(A), C_{1}=\psi(B)=\Psi(B)=\Psi[\Phi(A)]=F(A) .
$$

Mas, $C_{2}=f(A)=F(A), \operatorname{logo} C_{1}=C_{2}$.

\section{A.1.4 Logaritmo de Matriz}

Sejam $\lambda_{k}, k=1, \ldots, q \leq n$, os autovalores distintos de uma matriz $A, n \times n$. Suponha que $\lambda_{k} \neq 0, \forall k$. Retire do plano complexo uma semi-reta $\mathbf{r}$ que, comece na origem e não contenha nenhum dos autovalores de $A$.

Nesse plano sem a semi-reta podemos definir ramos de logaritmo, que denotaremos por $(\log (z))_{m}$, $m=0, \pm 1, \pm 2, \ldots$, por

$$
(\log (z))_{m}=\log (|z|)+i(\arg (z)+2 \pi m) .
$$

Aqui $\arg (z)$ é o argumento do número complexo $z$.

Sejam $\Gamma_{k}$ 's círculos centrado em $\lambda_{k}, k=1, \ldots, q$, dois a dois disjuntos que não intersectam a reta $\mathbf{r}$. Seja $G_{k}$ o disco aberto de bordo $\Gamma_{k}$ dois a dois disjuntos, e $G=\bigcup_{k} G_{k}$. Definimos função analítica por partes em $G$ por

$$
\log (z)=(\log (z))_{m_{k}}, z \in G_{k},(k=1, \ldots, q) .
$$

Usando a fórmula de Cauchy, podemos agora definir uma função analítica por partes $\log (A), A$ matriz $n \times n$, por

$$
\log (A)=\frac{1}{2 \pi i} \sum_{k=1}^{q} \oint_{\Gamma_{k}}(\xi I-A)^{-1} \log (\xi)_{m_{k}} d \xi .
$$

Queremos provar que a matriz $K=\log (A)$ satisfaz a equação

$$
e^{K}=A \text {. }
$$

Seja $\phi(z)=(\log (z))_{m_{k}}, z \in G_{k}, \psi(w)=e^{w}$, vemos que a função composta $f(z)=\psi(\phi(z))$ é simplesmente a identidade $f(z)=z$.

Pelo lema da composição de funções, o msmo resultado é obtido, seja por tomarmos diretamente $f(A)=A$ ou se primeiramente calcularmos $K=\log (A)$ e então $e^{K}=f(A)$, logo vale (A.5). Como se percebe, existe um número infinito de matrizes $K$ satisfazendo a igualdade (A.5), visto que os números $m_{k}$ são arbitrários.

Os autovalores da matriz $K=\log (A)$ são os números $\left[\log \left(\lambda_{k}\right)\right]_{m_{k}}$. De fato, seja $A v_{k}=\lambda_{k} v_{k}$, então $(\xi I-A)^{-1} v_{k}=\left(\xi-\lambda_{k}\right)^{-1} v_{k}$. Substituindo em (A.4) e usando a fórmula de Cauchy, temos

$$
(\log (A)) v_{k}=\left[\log \left(\lambda_{k}\right)\right]_{m_{k}} v_{k} .
$$

Sejam $\alpha_{k}$ números complexos arbitrários tais que $e^{\alpha_{k}}=\lambda_{k}$. Sempre existe uma matriz $K=$ $\log (A)$ com autovalores $\alpha_{k}$. Essa matriz é determinada por (A.4) com os valores $m_{k}$ escolhidos de acordo com as condições

$$
\alpha_{k}=\left[\log \left(\lambda_{k}\right)\right]_{m_{k}} .
$$




\section{Apêndice B}

\section{Algoritmos implementados}

1. Algoritmo usado para esboçar o espaço de configuração da massa infinitesimal.

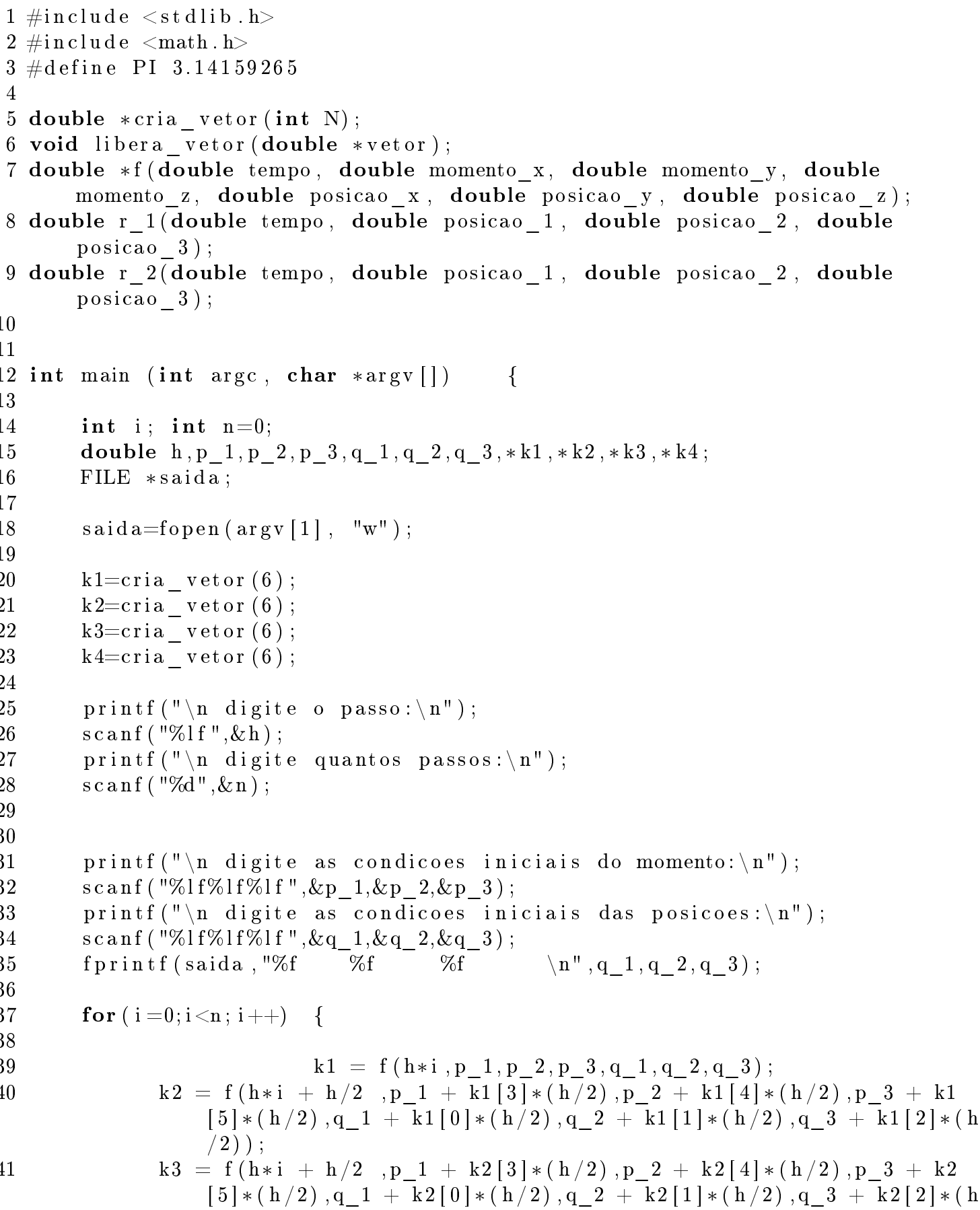


/2)) ;

42

43

44

45

46

47

48

49

50

51

52

53

54

55

56

57

58

59

$60\}$

61

62

63

64

65

66

67

68

69

$70\}$

71

74

75

76

77

78

79

80

81

82

$83\}$

84

85

86

8

$$
\begin{aligned}
\mathrm{k} 4= & \mathrm{f}\left(\mathrm{h} * \mathrm{i}+\mathrm{h} / 2, \mathrm{p} \_1+\mathrm{k} 3[3] *(\mathrm{~h}), \mathrm{p} \_2+\mathrm{k} 3[4] *(\mathrm{~h}), \mathrm{p} \_3\right. \\
& +\mathrm{k} 3[5] *(\mathrm{~h}), \mathrm{q} \_1+\mathrm{k} 3[0] *(\mathrm{~h}), \mathrm{q} \_-{ }^{2}+\mathrm{k} 3[1] *(\mathrm{~h}), \mathrm{q}_{-}{ }^{+}+ \\
& \mathrm{k} 3[2] *(\mathrm{~h}))
\end{aligned}
$$

$$
\begin{array}{r}
\mathrm{q} \_1=\mathrm{q} \_1+(\mathrm{h} / 6.0) *(\mathrm{k} 1[0]+2.0 * \mathrm{k} 2[0]+2.0 * \mathrm{k} 3[0]+\mathrm{k} 4[0]) ; \\
\mathrm{q} \_2=\mathrm{q} \_2+(\mathrm{h} / 6.0) *(\mathrm{k} 1[1]+2.0 * \mathrm{k} 2[1]+2.0 * \mathrm{k} 3[1]+\mathrm{k} 4[1]) ; \\
\mathrm{q} \_3=\mathrm{q} \_3+(\mathrm{h} / 6.0) *(\mathrm{k} 1[2]+2.0 * \mathrm{k} 2[2]+2.0 * \mathrm{k} 3[2]+\mathrm{k} 4[2]) ; \\
\mathrm{p} \_1=\mathrm{p} \_1+(\mathrm{h} / 6.0) *(\mathrm{k} 1[3]+2.0 * \mathrm{k} 2[3]+2.0 * \mathrm{k} 3[3]+\mathrm{k} 4[3]) ; \\
\mathrm{p} \_2=\mathrm{p} \_2+(\mathrm{h} / 6.0) *(\mathrm{k} 1[4]+2.0 * \mathrm{k} 2[4]+2.0 * \mathrm{k} 3[4]+\mathrm{k} 4[4]) ; \\
\mathrm{p} \_3=\mathrm{p}-3+(\mathrm{h} / 6.0) *(\mathrm{k} 1[5]+2.0 * \mathrm{k} 2[5]+2.0 * \mathrm{k} 3[5]+\mathrm{k} 4[5]) ;
\end{array}
$$

libera vetor $(\mathrm{k} 1)$;

libera_vetor $(\mathrm{k} 2)$;

libera_vetor $(\mathrm{k} 3)$;

libera_vetor $(\mathrm{k} 4)$;

return $(0)$;

\}

*função que desaloca memoria do vetor do arquivo*/

void libera_vetor (double *vetor) $\quad$ (

free (vetor);

vet or $=$ NULL;

*

*função que aloca memoria da matriz do arquivo*/

double $*$ cria_vetor $($ int $\mathrm{N}) \quad\{$

double $*$ vet;

vet $=($ double $*)$ malloc $(\mathrm{N} *$ si z e of $($ double $*))$;

if $($ vet $=$ NULL $)$ \{

printf("nao foi possivel alocar memoria");

exit (1) ;

\}

return vet;

(1)

/*função do movimento*/

void $*$ /double $* \mathrm{f}$ (double tempo, double momento_1, double momento_2, double momento_3, double posicao_1, double posicao_ 2 , double posicao_3)

double *vet;

vet $=$ cria_

vet $[0]=\overline{m o m e n t o}_{-} 1+$ posicao_ 2 ;

$\operatorname{vet}[1]=$ momento $2-$ posicao 2 ;

$\operatorname{vet}[2]=$ momento_3;

$\operatorname{vet}[3]=$ momento_2 $-(0.5) *\left(\left((\right.\right.$ posicao_ $1+(0.5)) /$ pow $\left(\mathrm{r}_{-} 1(\right.$ tempo, posicao_1, posicao_2, posicao_3),3) $)+\left((\right.$ posicao__ $-(0.5)) /$ pow $\left(\mathrm{r}_{-} 2\right.$ (tempo, posicao_1, posicao_2, posicao_3),3)) );

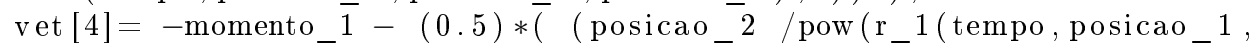

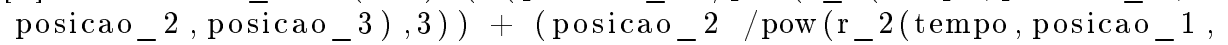
posicao_2, posicao_3),3)) );

$\operatorname{vet}[5]=-(\bar{p}$ osicao_ $3 / \overline{2}) *\left(\left(1 /\right.\right.$ pow $\left(\mathrm{r}_{-} 1(\right.$ tempo, posicao_ 1, posicao_ 2 ,

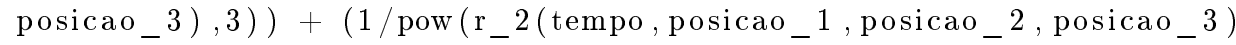
,3))); 


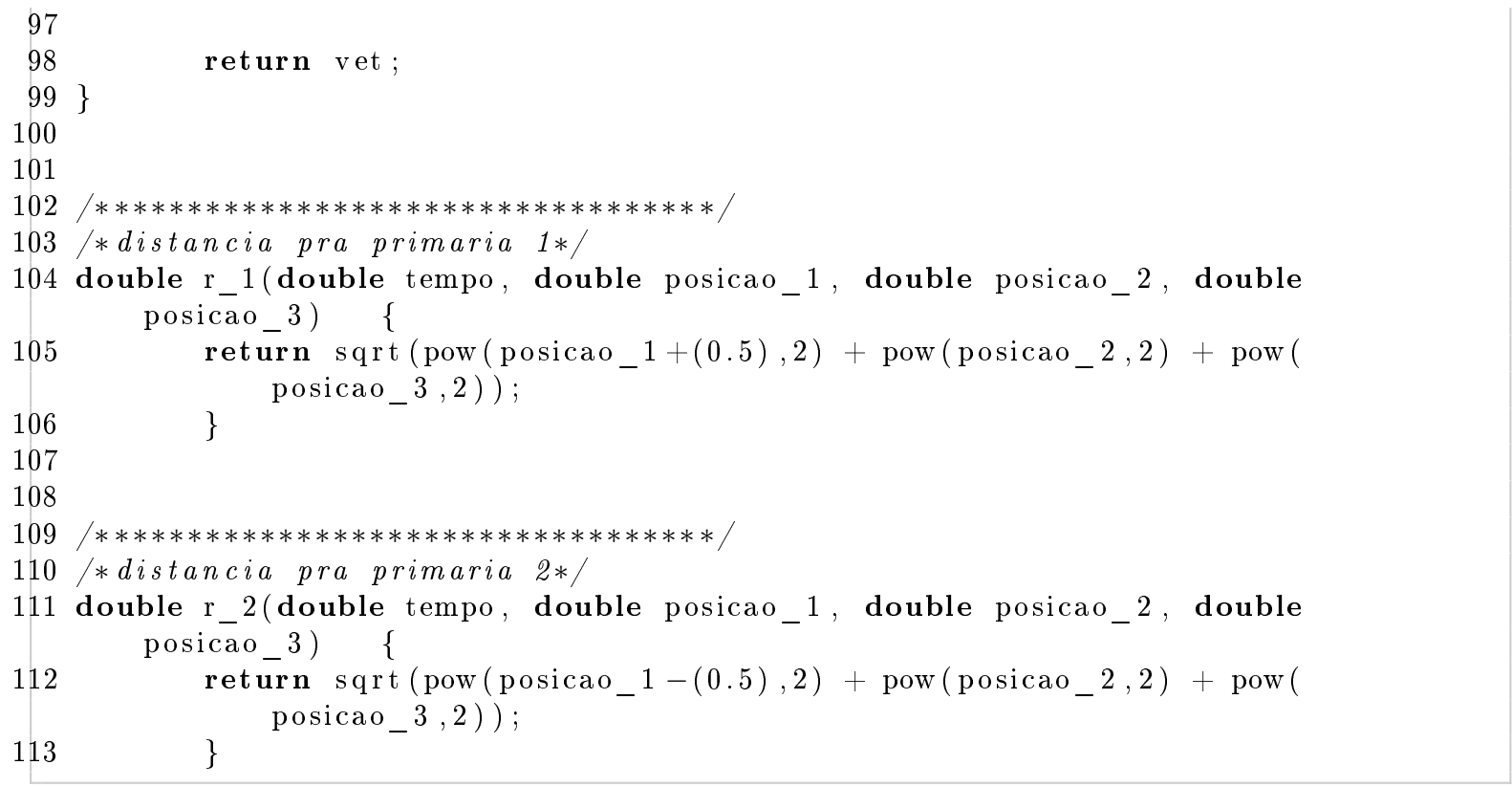

2. Algoritmo usado para achar os autovalores aproximados da matriz de monodromia.

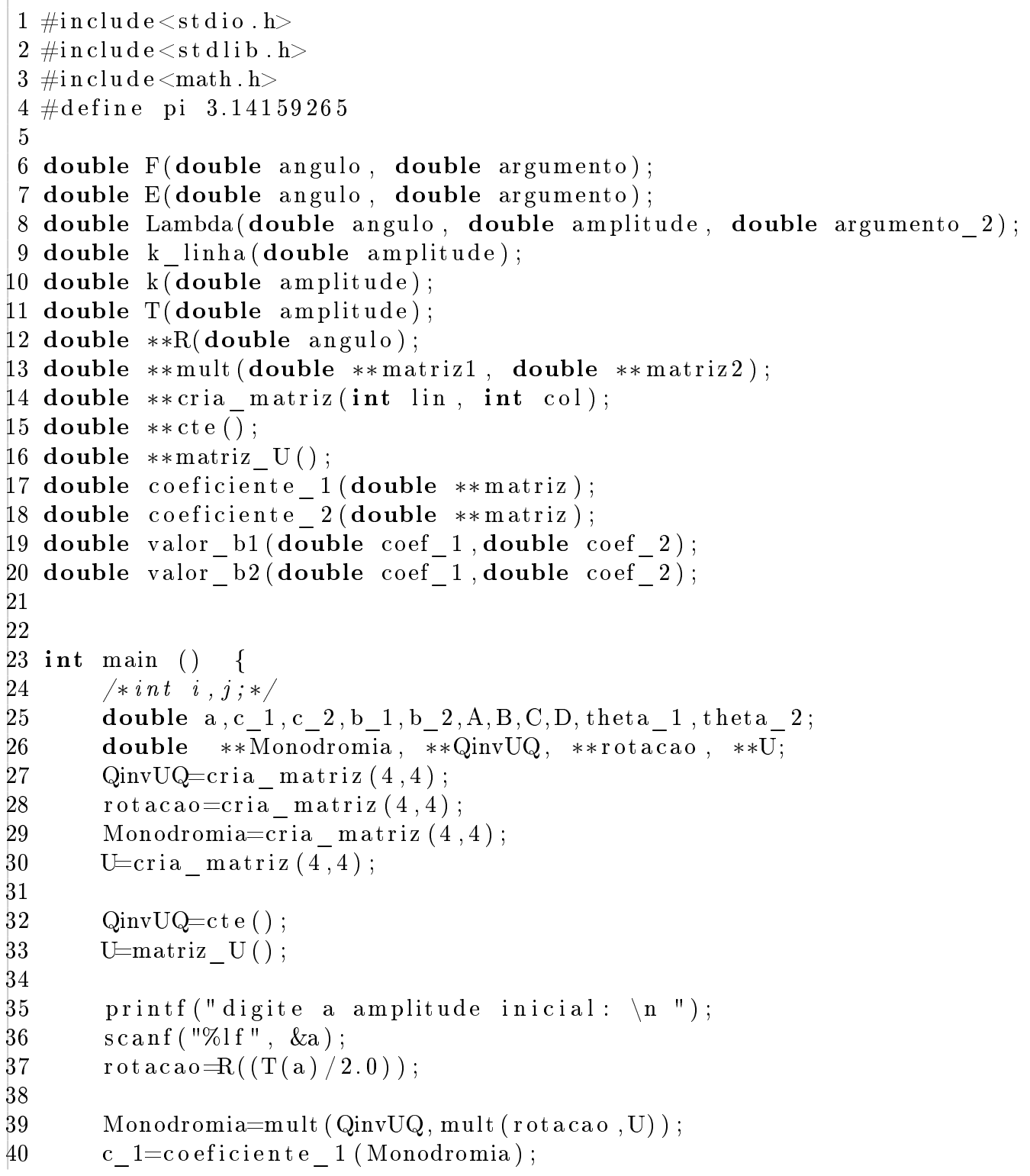




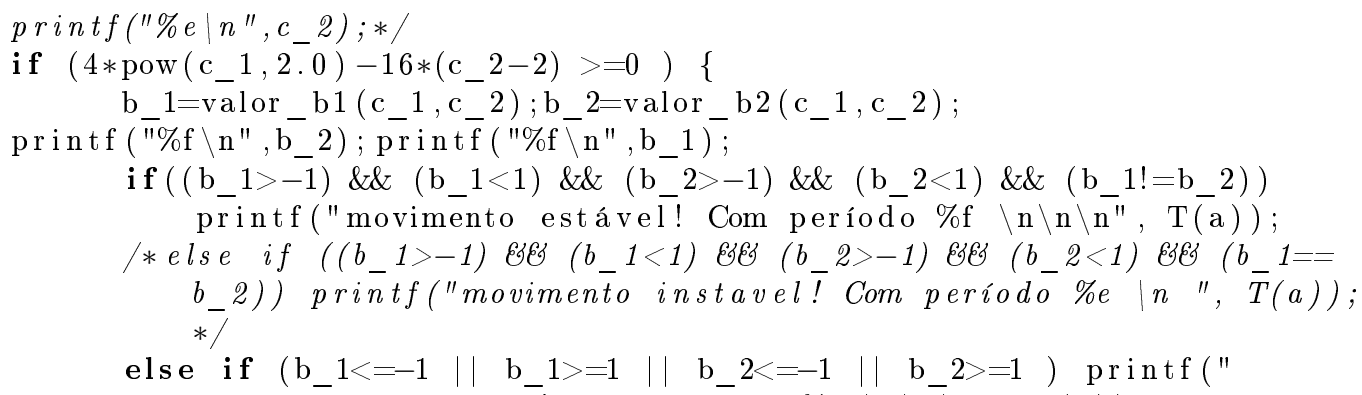




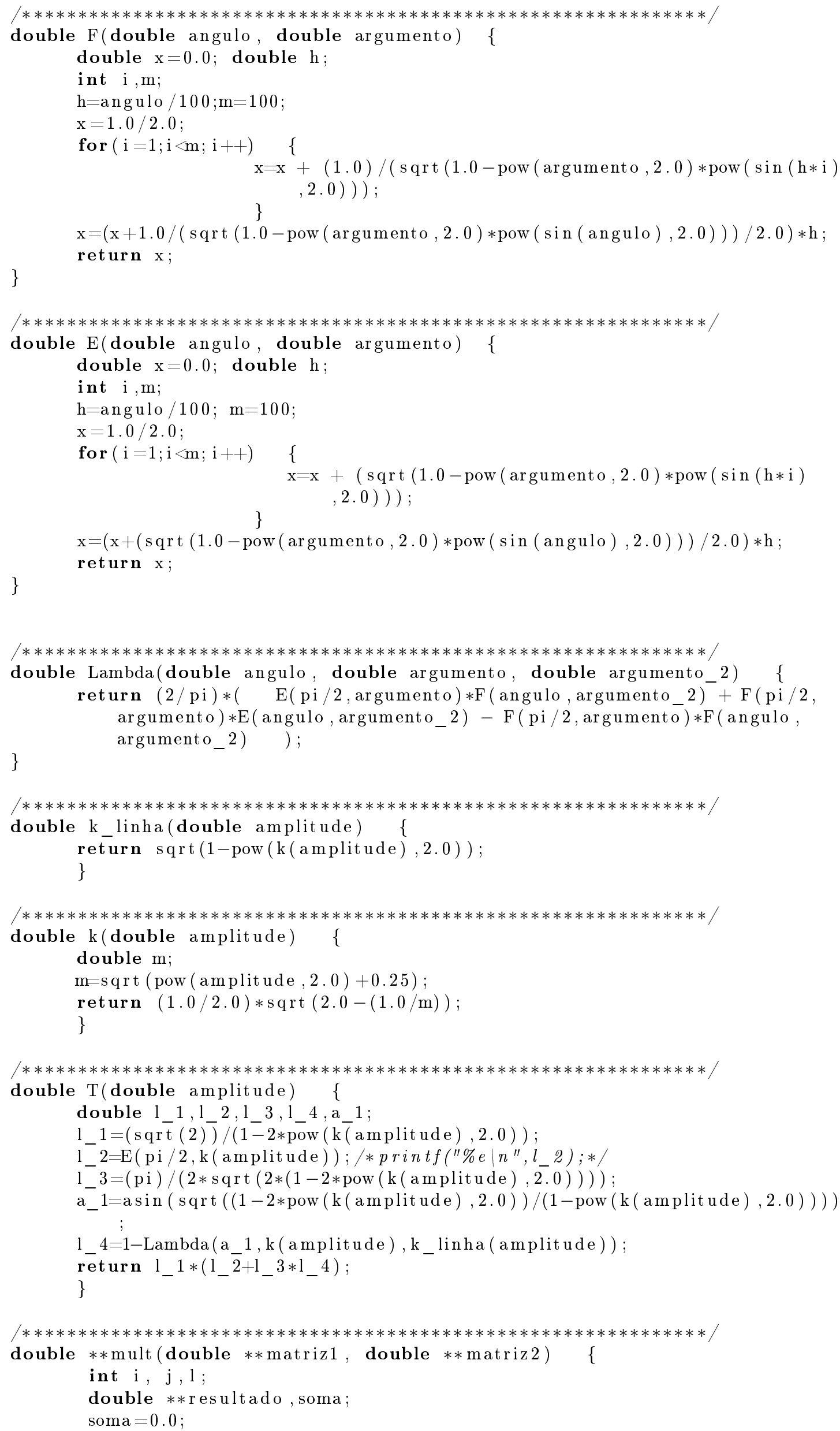

double Lambda(double angulo, double argumento, double argumento_2) \{ return $(2 / \mathrm{pi}) *(\mathrm{E}(\mathrm{pi} / 2$, argumento $) * \mathrm{~F}($ angulo, argumento_ 2$)+\mathrm{F}(\mathrm{pi} / 2$, argumento $) * \mathrm{E}($ angulo, argumento_2 $)-\mathrm{F}(\mathrm{pi} / 2$, argumento $) * \mathrm{~F}($ angulo, argumento_2) ); 
resultado $=$ cria_matriz $(4,4)$;

for $(1=0 ; 1<4 ; 1+\overline{+}) \quad\{$ for $(i=0 ; i<4 ; i++) \quad\{$

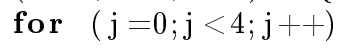




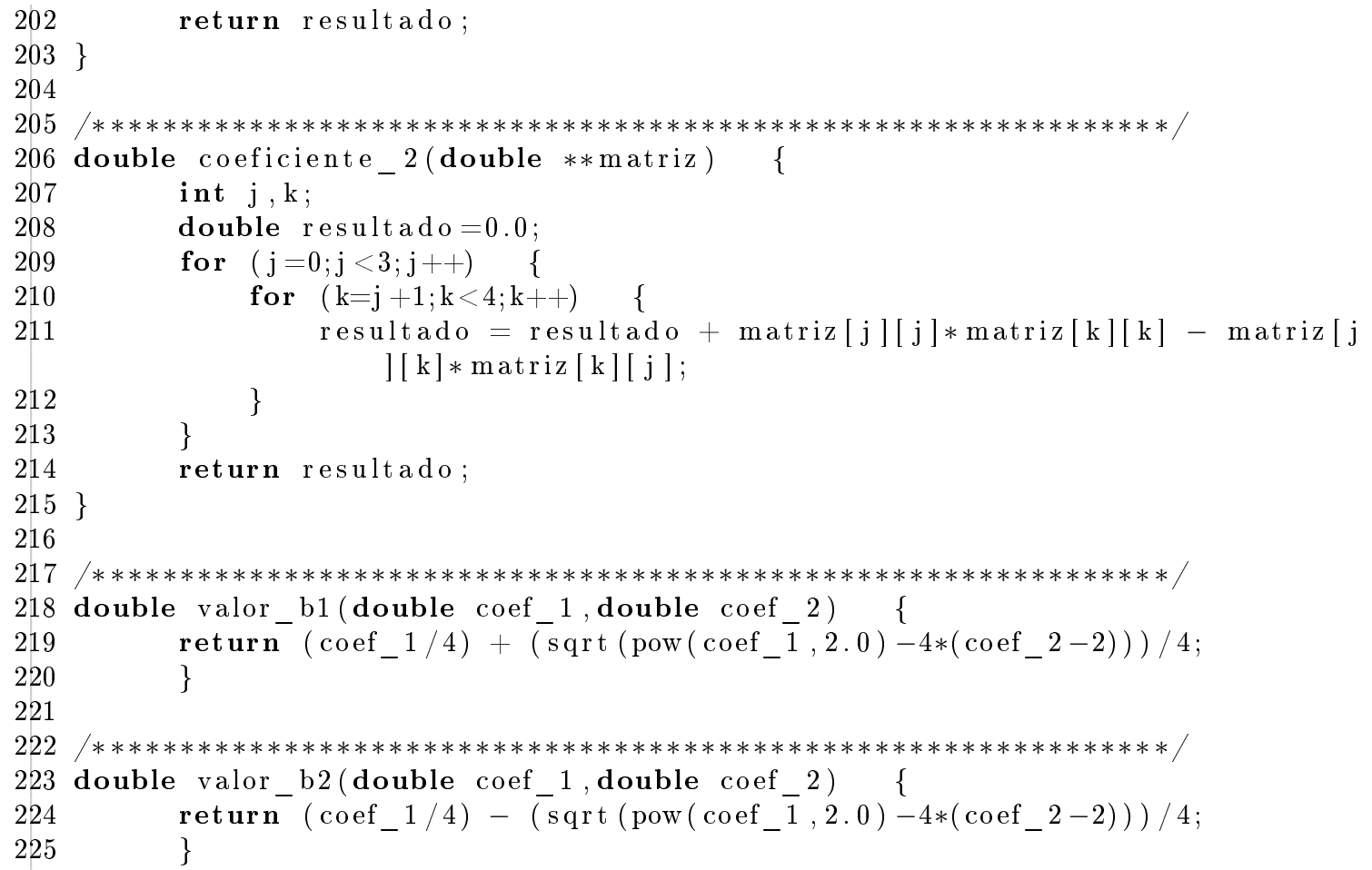


APÊNDICE B 


\section{Referências Bibliográficas}

[BF71] Paul F. Byrd e Morris D. Friedman. Handbook of elliptic integrals for engineers and scientists. Die Grundlehren der mathematischen Wissenschaften, Band 67. SpringerVerlag, New York, 1971. Second edition, revised. 37, 38

[BLO94] Edward Belbruno, Jaume Llibre, e Merce Ollé. On the families of periodic orbits which bifurcate from the circular Sitnikov motions. Celestial Mech. Dynam. Astronom., 60(1):99129, 1994. 3, 35, 37, 39

[BP09] T. Bountis e K. E. Papadakis. The stability of vertical motion in the $N$-body circular Sitnikov problem. Celestial Mech. Dynam. Astronom., 104(1-2):205-225, 2009. 48, 51

[GR97] Clodoaldo Grotta Ragazzo. On the stability of double homoclinic loops. Comm. Math. Phys., 184(2):251-272, 1997. 3

[Lan81] R. G. Langebartel. Formulation of the restricted three-body problem with two-center problem orbits as intermediate orbits. Astrophys. and Space Sci., 75(2):437-454, 1981. 38

[Mey99] Kenneth R. Meyer. Periodic solutions of the $N$-body problem, volume 1719 of Lecture Notes in Mathematics. Springer-Verlag, Berlin, 1999. 3, 19

[MHO09] Kenneth R. Meyer, Glen R. Hall, e Dan Offin. Introduction to Hamiltonian dynamical systems and the $N$-body problem, volume 90 of Applied Mathematical Sciences. Springer, New York, second edição, 2009. 5

[Per07] E. A. Perdios. The manifolds of families of 3D periodic orbits associated to Sitnikov motions in the restricted three-body problem. Celestial Mech. Dynam. Astronom., 99(2):85104, 2007. 35

[PM88] E. Perdios e V. V. Markellos. Stability and bifurcations of Sitnikov motions. Celestial Mech., 42(1-4):187-200, 1987/88. 3, 35, 51

[SBD07] P. Soulis, T. Bountis, e R. Dvorak. Stability of motion in the Sitnikov 3-body problem. Celestial Mech. Dynam. Astronom., 99(2):129-148, 2007. xiii, 35, 36, 46, 47, 51

[Sid11] Vladislav V. Sidorenko. On the circular Sitnikov problem: the alternation of stability and instability in the family of vertical motions. Celestial Mech. Dynam. Astronom., 109(4):367-384, 2011. 35, 36, 48, 51

[Sit60] K. Sitnikov. The existence of oscillatory motions in the three-body problems. Soviet Physics. Dokl., 5:647-650, 1960. 2

[Sze67] V. Szebehely. Theory of orbits. The restricted problem of three bodies. 1967. 19

[YS75] V. A. Yakubovich e V. M. Starzhinskii. Linear differential equations with periodic coefficients. 1, 2. Halsted Press [John Wiley \& Sons] New York-Toronto, Ont.,, 1975. Translated from Russian by D. Louvish. 5, 9, 11, 12, 13, 14, 15 